Rev. Elev. Méd. vét. Pays trop., 1975, 28 (3) : 331-403.

\title{
Les organes génitaux de la femelle Zébu
}

\author{
par P. CUQ (*) et K. M. AGBA $\left(^{* *}\right)$
}

\section{RESUME}

Les organes génitaux de la femelle zébu sont décrits en détail. Les auteurs soulignent les différences anatomiques existant par rapport à Bos taurus.

\section{TABLE DES MATIERES}

Anatomie macroscopique des ovaires

I. Morphologie .

I.A. Conformation .

I.B. Dimensions et poids

I.C. Variations .

II. Moyens de fixité .

II.A. Le mésovarium

II.B. Le mésosalpinx

II.C. La bourse ovarique .

II.D. Les ligaments de l'ovaire

III. Topographie et rapports . . . . . . . . . . . . . . . . . . . . 342

III.A. Topographie et rapports des ovaires de la génisse . . . . . . . . 342

III.B. Topographie et rapports des ovaires de la vache gestante . . . . . 342

III.C. Topographie et rapports des ovaires de la vache multipare . . . . 342

(*) Professeur à l'Ecole Inter-Etats des Sciences et Médecine Vétérinaires, Université de Dakar (Rép. du Sénégal).

(*) Assistant à l'Ecole Inter-Etats des Sciences e Médecine Vétérinaires, Université de Dakar (Rép. du 
IV. Vascularisation - Innervation

IV.A. L'artère ovarienne . . . . . . . . . . . . . . . . . . 344

IV.B. La veine ovarienne . . . . . . . . . . . . . . . . . . . . . . 344

IV.C. Les lymphatiques de l'ovaire . . . . . . . . . . . . . . . . 344

IV.D. Les nerfs de lovaire . . . . . . . . . . . . . . 345

Anatomie microscopique des ovaires . . . . . . . . . . . . . . . . . 345

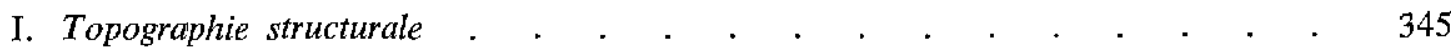

I.A. L'épithélium ovarien . . . . . . . . . . . . . . . . 345

I.B. Le stroma ovarien . . . . . . . . . . . . . . . 345

I.C. Les vaisseaux et les nerfs intra-ovariens . . . . . . . . . . . 347

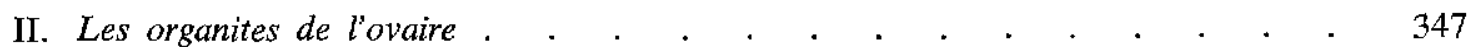

II.A. Les follicules évolutifs . . . . . . . . . . . . . . . . . 347

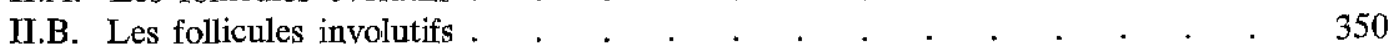

II.C. Les corps progestatifs et gestatifs . . . . . . . . . . . 354

II.D. Les périodes de repos sexuel ou anoestrus . . . . . . . . . $\quad$. 358

CHAPITRE DEUXIEME : PORTION TUBULAIRE OU GESTATIVE DES ORGANES GENITAUX .

Les oviductes . . . . . . . . . . . . . . . . . . . . 360

I. Morphologie . . . . . . . . . . . . . . . . . 360

I.A. Conformation extérieure . . . . . . . . . . . . . 360

I.B. Conformation intérieure . . . . . . . . . . . . . . 361

II. Moyens de fixité. Topographie. Rapports ․ . . . . . . . . . . 362

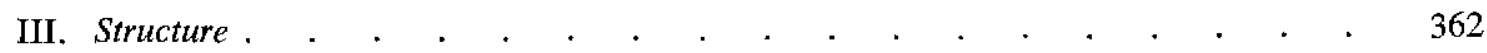

III.A. La tunique séreuse . . . . . . . . . . . . . . . . . 362

III.B. La tunique musculeuse . . . . . . . . . . . . . . 362

III.C. La tunique muqueuse . . . . . . . . • • • • •

IV. Vaisseaux et nerfs . . . . . . . . . . . . . . . . . . 365

IV.A. Les artères de l'oviducte . . . . . . . . . . . . . . . . 365

IV.B. Les veines tubaires . . . . . . . . . . . . . . . . . . 366

IV.C. Les lymphatiques de l'oviducte. . . . . . . . . . . . . . . 367

IV.D. Les netfs de l'oviducte . . . . . . . . . . . . . . . . 367

L'utérus . . . . . . . . . . . . . . . . . . . 367

I. Morphologie . . . . . . . . . . . . . . . . . . . 367

I.A. Conformation extérieure . . . . . . . . . . . . . . 367

I.B. Conformation intérieure . . . . . . . . . . . . . 371

I.C. Le col utérin . . . . . . . . . . . . . . . 372

II. Moyens de fixité, rapports et topographie . . . . . . . . . . . . . . 373

II.A. Le ligament large . . . . . . . . . . . . . . . 373

II.B. Topographie et rapports de l'utérus . . . . . . . . . . . 375

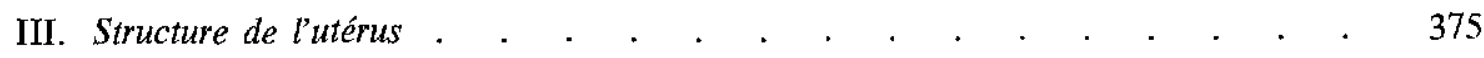

III.A. La tunique séreuse . . . . . . . . . . . . . . . . . . . . . . 376

III.B. La tunique musculeuse . . . . . . . . . . . . . . . . . 376

III.C. La tunique muqueuse . . . . . . . . . . . . . . . . . 378 
IV. Irrigation et innervation de l'utérus

IV.A. Les artères de l'utérus .

IV.B. Les veines de l'utérus.

IV.C. Les lymphatiques utérins

IV.D. Les nerfs de l'utérus

I. Morphologie et structure de la portion copulatrice.

I.A. Le vagin

I.B. Le vestibule vaginal

I.C. La vulve

II. Moyens de fixité de la portion copulatrice . . . . . . . . . . . . . . . 392

II.A. Les culs-de-sac pelviens du péritoine . . . . . . . . . . 392

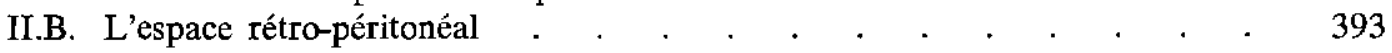

III. Irrigation et innervation de la portion copulatrice . . . . . . . . . . . . 393

III.A. Artères de la portion copulatrice . . . . . . . . . . . . . . . . 393

III.B. Veines de la portion copulatrice . . . . . . . . . . . . . . 395

III.C. Les lymphatiques de la portion copulatrice . . . . . . . . . 396

III.D. Les nerfs de la portion copulatrice . . . . . . . . . . . . . 396

\section{TABLE DES ILLUSTRATIONS}

1. L'ovaire et l'oviducte droits de la femelle zébu (vue médiale)

2. Topographie des organes génitaux de la femelle zébu primipare (vue demischématique latérale gauche après ablation du ligament large, de la corne utérine, de l'oviducte et de l'ovaire gauches)

3. Topographie des organes génitaux de la femelle zébu multipare (vue demischématique latérale gauche après ablation du ligament large, de la corne utérine, de l'oviducte et de l'ovaire gauches)

4. Histologie topographique de l'ovaire de la femelle zébu (représentation demischématique) .

5.1. Structure de l'ovaire de la femelle zébu . . . . . . . . . . . . . . . 352

5.2. Structure de l'ovaire de la femelle zébu . . . . . . . . . . . . . 353

5.3. Structure de l'ovaire de la femelle zébu . . . . . . . . . . . . . 355

5.4. Structure de l'ovaire de la femelle zébu . . . . . . . . . . . . . . . 359

6. L'oviducte et la corne utérine gauches de la femelle zébu (vue latérale) . . . 361

7. Structure de l'oviducte et de l'utérus de la femelle zébu . . . . . . . 364

8. Les artères de l'ovaire et de l'oviducte de la femelle zébu (vue demi-schématique.

9. Conformation extérieure des organes génitaux de la femelle zébu (vue ventrale) 36

10. Le tractus génital de la femelle zébu (vue latérale droite, organes en place, après ouverture du bassin).

11. Structure de l'utérus, du vagin et de la vulve de la femelle zébu . 
12. Les artères du tractus génital de la femelle zébu (vue demi-schématique latérale

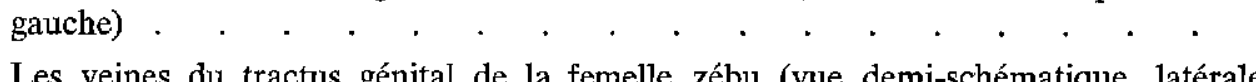

13. Les veines du tractus génital de la femelle zébu (vue demi-schématique, latérale gauche)

14. Conformation intérieure du tractus génital de la femelle zébu (vue dorsale, après ouverture du plafond des portions gestative et copulatrice)

15. Les artères et les nerfs du tractus génital de la femelle zébu (vue latérale droite, organes en place, après ouverture du bassin) organes en place, après ouverture du bassin). 


\title{
PREFACE
}

Il est toujours agréable de présenter un ou vrage de qualité qui vient à son heure. Le travail que publie aujourd'hui mon collègue et ami le Professeur P. Cuq, Chef du Département d'Anatomie, Histologie et Embryologie de l'Ecole Inter-Etats des Sciences et Médecine Vétérinaires de Dakar, est de ceux-là.

Jusqu'à ce jour, les spécialistes de l'élevage du zébu : étudiants, vétérinaires, zootechniciens, praticiens ou chercheurs, n'avaient aucun document de référence concernant l'anatomie du zébu. Or le zébu constitue, avec les petits ruminants, le moyen de survie de la majorité des populations qui vivent en zone inter-tropicale aride. L'augmentation nécessaire des effectifs se heurte depuis toujours au facteur limitant de la reproduction, qui, dans cette espèce, connaît des intermittences ou des fléchissements, source de pertes considérables.

Connaitre mieux le zébu, et plus particulièrement les phénomènes complexes qui président à sa reproduction a été la motivation du Professeur Cuq qui, dès son arrivée à Dakar, s'est attaché à élucider les particularités anatomiques et fonctionnelles propres à cette espèce. C'est dire l'intérêt primordial qui s'attache à ce travail didactique "Les organes génitaux de la femelle zébu », présenté ici sous forme de fascicule et premier, je le souhaite, d'une longue série.

Présenter un ouvrage d'anatomie est pour le non-spécialiste une redoutable épreuve. Mais la conception moderne de l'anatomie, à laquelle le Professeur Cuq est profondément attaché, rend ma tâche plus aisée. En effet, si les résultats des recherches sont présentés dans la forme familière de l'anatomie descriptive, macroscopique et microscopique, les notions moins traditionnelles d'anatomie topographique, évolutive et fonctionnelle sont évoquées largement. C'est dire que l'anatomie présentée est celle qui doit être enseignée dans une Ecole Vétérinaire moderne, c'est-à-dire une anatomie appliquée.

L'appareil génital de la femelle se prêtait merveilleusement bien à cette conception. L'anatomie n'est plus cette science figée qu'était l'anatomie du cadavre. Les structures de l'organisme, voire les rapports des organes, évoluent dans le temps. A partir de l'étude morphologique, mais surtout de l'examen histologique, il est possible d'expliquer les phénomènes physiologiques et même pathologiques. Pour avoir eu l'honneur d'être associé à une partie de ces travaux, j’ai pu constater que tel est bien l'esprit avec lequel le Professeur Cuq a mené cette étude portant sur plusieurs centaines d'animaux.

Ce travail d'équipe, qui représente l'essentiel des efforts de recherche de tout le laboratoire d'anatomie depuis sa création, n'a pu voir le jour que grâce au soutien moral et à l'aide matérielle dispensés à la jeune Ecole par ses tuteurs : l'Université de Dakar en la personne de son Recteur, le Professeur Seydou Madani Sy, dont la sympathie ne s'est jamais démentie, le Fonds d'Aide et de Coopération, ainsi que les Etats de l'Afrique francophone, attachés au fonctionnement de l'Ecole, dont les contributions financières ont permis de mener à bien les investissements nécessaires, la Revue d'Elevage et de Médecine vétérinaire des Pays tropicaux et son rédacteur en chef le Dr Sauvel, grâce à qui cette publication paraît, sous une présentation qui en fait tout l'intérêt pour une large diffusion. Qu'ils soient tous ici remerciés pour leur aide et leur collaboration.

Cet ouvrage doit être pour les étudiants un outil de travail adapté à leurs besoins et pour les vétérinaires exerçant en zone tropicale, pathologistes ou zootechniciens du zébu, une référence de qualité. C'est pourquoi je suis certain qu'il sera accueilli avec la faveur qu'il mérite, par tous les spécialistes et qu'il pourra témoigner ainsi de la valeur du travail accompli à l'Ecole de Dakar.

\author{
Professeur J. FERNEY, \\ Directeur de l'Ecole Inter-Etats \\ des Sciences et Médecine vétérinaires de Dakar.
}




\section{INTRODUCTION}

Les importantes analogies morphologiques et structurales qui rapprochent les deux espèces domestiques du genre $B o s$ (Bos taurus et Bos indicus) sont à l'origine d'une regrettable confusion qui pose en principe l'identité fondamentale de l'anatomie et du fonctionnement de leurs organes.

LES ORGANES GENITAUX DE LA FEMELLE ZEBU (Organa genitalia feminina Bos indicus) n'ont pas échappé à cette généralisation hâtive. Ils sont habituellement considérés comme anatomiquement identiques à ceux des bovins des régions tempérées et les particularités fonctionnelles qu'ils présentent sont considérées dans leur ensemble comme les conséquences de l'adaptation de l'espèce aux dures conditions d'alimentation et d'environnement des régions tropicales sèches.

Nombre de différences anatomiques et structurales, telles celles de la région cervicofornicale et certaines formes particulières d'activité ou d'involution des organites ovariens, correspondent cependant à des caractères nettement spécifiques.

Le but de ce travail est d'en dresser le bilan dans le domaine de l'Anatomie macroscopique et microscopique. Avec la thèse de Doctorat-Vétérinaire de l'un d'entre nous, il représente la seule étude complète sur le sujet. Nous souhaitons qu'il puisse servir de base à des recherches plus directement orientées vers l'exploitation rationnelle du zébu qui ne doit pas être nécessairement effectuée selon les normes et les méthodes admises pour les bovins des régions tempérées.

L'étude de base pour la préparation de ce document a porté sur la dissection des tractus génitaux de 49 femelles, d'âges et d'états physiologiques divers, toutes de races Gobra et Maure ou issues du croisement des deux. La partie microscopique a nécessité des prélèvements des diverses sections du tractus génital qui ont été effectués sur les animaux précédents et sur des sujets sacrifiés pour la boucherie aux abattoirs de Dakar. Pour la seule étude structurale, les gonades et les tractus génitaux de 284 femelles ont été examinés.

En raison des problèmes que soulève l'adoption par la «francophonie » d'une traduction de la Nomenclature internationale, nous nous sommes efforcés de choisir parmi tous les synonymes possibles ceux qui peuvent être compris sans ambiguité par les utilisateurs du français de toutes nationalités et par tous ceux qui s'intéressent à l'élevage de cette espèce sans être pour autant spécialistes d'anatomie. Dans tous les cas, nous avons en outre pris soin de faire suivre le terme français du terme latin de la liste officielle des Nomina Anatomica Veterinaria publiés en 1973. Pour l'histologie, nous nous sommes référés à la liste encore officieuse des Nomina Histologica Veterinaria que prépare l'Association Mondiale des Anatomistes Vétérinaires.

Nous tenons à remercier ici tous ceux qui nous ont aidés dans la réalisation de ce travail. Monsieur le Professeur Seydou Sy, Recteur de I'Université de Dakar, Monsieur Ie Professeur Jean Ferney, Directeur de l'Ecole Inter-Etats des Sciences et Médecine vétérinaires, qui nous ont permis sa réalisation et sa diffusion, les assistants français, volontaires du Service National : MM. Van Craeynest, Vernizy, Cordier, Loeillot et Renner, qui depuis 1969 ont tour à tour participé à l'activité du laboratoire, Monsieur Moussa Diop qui avec son talent habituel a réalisé les planches d'illustration et tous les étudiants des Etats francophones d'Afrique tropicale qui se sont succédés depuis 1968 autour des tables de dissection et ont ainsi contribué, sans s'en rendre compte, à l'élaboration progressive de ce document.

Il nous est particulièrement agréable de faire une mention spéciale pour notre collègue et Directeur, le Professeur Jean Ferney qui malgré les lourdes charges de ses fonctions enseignantes et administratives, a bien voulu rédiger la préface de ce document. Qu'il trouve ici l'expression de notre reconnaissance.

Il nous est agréable aussi d'exprimer enfin nos remerciements au Docteur Sauvel, Rédacteur en Chef de la Revue d'Elevage et de Médecine vétérinaire des Pays tropicaux qui avec son dévouement habituel à la cause des Pays en voie de développement a bien voulu se charger de la publication et de la diffusion de ce travail. 
Respectant le plan classique de l'anatomie descriptive, l'exposé sera divisé en trois chapitres consacrés successivement à la portion glandulaire, à la portion tubulaire ou gestative et la portion copulatrice des organes génitaux de la femelle.

\section{Chapitre I \\ Portion glandulaire des organes génitaux de la femelle Zébu}

Les OVAIRES (Ovaria) correspondent à la SECTION GLANDULAIRE des ORGANES GENITAUX DE LA FEMELLE (Organa genitalia feminina). Leur fonction est double, à la fois gamétogène et endocrine.

La FONCTION GAMETOGENE se traduit par la maturation et la libération d'OVULES qui dégénèrent ou, après fécondation, se transforment en CEUFS qui se fixent dans l'utérus et s'y développent.

La FONCTION ENDOCRINE, par la sécrétion d'HORMONES qui la caractérise, régit l'activité du reste de l'appareil génital. Elle est intimement liée à la fonction gamétogène.

En l'absence de fécondation, les deux fonctions se reproduisent périodiquement. L'ensemble des phénomènes dont la succession caractérise une période constitue le CYCLE OESTRAL.

Un cycle se subdivise hormonalement en deux périodes : LA PERIODE OESTROGENIQUE au cours de laquelle un follicule murit (Pro-oestrus) puis libère son ovule (Oestrus) et LA PERIODE PROgeSTATIVE caractérisée par le développement (Post-oestrus) puis le début de la régression (Dioestrus) d'un corps progestatif ou CORPS JAUNE CYCLIQUE (Corpus luteum cyclicum) qui se développe à partir du follicule après l'ovulation. Lorsque les cycles se suivent de façon ininterrompue pendant toute l'année, la femelle est dite à activité sexuelle continue. Dans les conditions habituelles de l'élevage en zone soudano-sahélienne de l'Afrique tropicale de l'Ouest, la femelle Zébu présente fréquemment des périodes de REPOS SEXUEL appelées Anoestrus. Son activité sexuelle est alors dite discontinue.

Lors de la gestation, c'est encore l'ovaire qui, par action hormonale, provoque l'arrêt de la succession des cycles et parachève la préparation de la muqueuse utérine à la fixation de l'œuf et à son développement. L'hormone est sécrétée par un corps jaune PARTICULIER dit CORPS GESTATIF ou CORPS JAUNE DE GESTATION (Corpus luteum graviditatis) qui persiste jusqu'à la parturition chez Bos taurus et, chez Bos indicus, peut être remplacé, supplémenté ou disparaître complètement.

Nous étudierons successivement les caractères anatomiques macroscopiques et microscopiques des ovaires.

\section{ANATOMIE MACROSCOPIQUE DES OVAIRES}

Placés à l'extrémité craniale du tractus génital, les ovaires (Ovaria) sont appendus à l'entrée du bassin par la portion rostrale des ligaments larges.

Nous exposerons successivement leurs caractéristiques morphologiques, séro-ligamenteuses, topographiques et vasculo-nerveuses. 


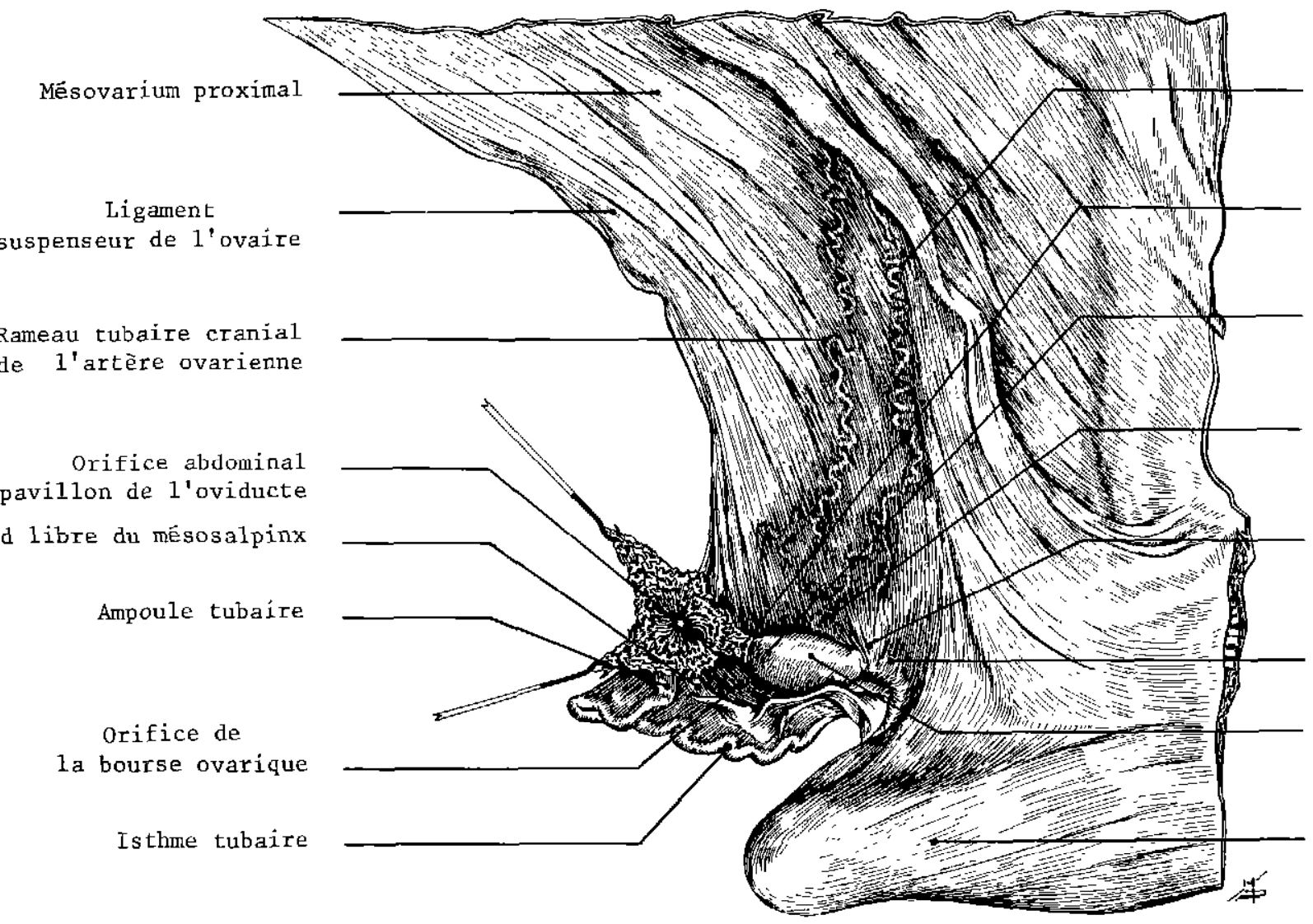

Artēre ovarienne

Ligament tubo-ovarique

Extrémité tubaire de l'ovaire

Mésovarium distal

Extrëmitê utêrine de 1'ovaire

Ligament propre de l'ovaire

Face médiale de 1 'ovaire

Corne utérine droite

L'ovaire et $1^{\prime}$ oviducte droits de la femelle zébu (vue médiale) 


\section{MORPHOLOGIE DES OVAIRES}

Morphologiquement, l'ovaire de Bos indicus ressemble à celui de Bos taurus. Il en diffère cependant par quelques caractères qui se rapportent à la conformation, au poids et aux dimensions de la glande.

\section{I.A.) Conformation}

Chez la femelle adulte, la glande est de coloration jaunâtre plus ou moins rosée. Elle a généralement la forme d'une amande aplatie latéro-médialement.

Son gros pôle ou EXTREMITE TUBAIRE (Extremitas tubaria) est en rapport avec le pavillon de l'oviducte. Il est orienté cranialement.

Son petit pôle ou EXTREMITE UTERINE (Extremitas uterina) est proche de 1'extrémité rostrale de la corne utérine. Il est orienté caudalement.

SOn BORD DORSAL ou BORD MESOVARIEN (Margo mesovaricus) reçoit l'insertion du mésovarium distal. Dans la plus grande partie de son étendue, il correspond au HILE de l'OVAIRE (Hilus ovarii) et livre passage aux vaisseaux et aux nerfs de la glande.

Son BORD VENTRAL ou BORD LIBRE (Margo liber) limite dorsalement l'orifice de la bourse ovarique.

Ses faces, classiquement distinguées en FACE MEDIALE (Facies medialis) et FACE LA. TERALE (Facies lateralis) sont convexes et plus ou moins bosselées par des organites superficiels en cours d'évolution. Elles sont aussi marquées par des taches grisâtres et irrégulières, d'aspect dense, dans les zones où des ovulations se sont produites.

La face latérale est toujours libre. Elle est orientée vers la cavité de la bourse ovarique et mérite le nom de face antrale (Facies antralis).

La face médiale est en rapport avec la cavité abdomino-pelvienne et les viscères digestifs de la région. Elle pourrait recevoir le nom de face viscérale (Facies visceralis). Cette face est très souvent recouverte, en partie ou en totalité, par le feuillet séreux interne du mésovarium distal. Ce recouvrement péritonéal déborde parfoís largement sur le bord ventral de la glande qui devient fixe tandis que le bord dorsal devient libre.

Assez fréquemment, l'ovaire du zébu ne se présente pas sous la forme caractéristique que nous venons de décrire. Il est alors grossièrement ovalaire, avec des bords presque parallèles et des pôles subégaux.

\section{I.B.) Dimensions et poids}

L'ovaire du zébu est plus petit et moins lourd que celui des bovins des régions tempérées.

Lorsqu'il ne contient pas d'organite volumineux, ses dimensions moyennes sont : longueur 26 à $28 \mathrm{~mm}(17$ à $37 \mathrm{~mm})$, hauteur 17 à $18 \mathrm{~mm}(9$ à $26 \mathrm{~mm})$, épaisseur 11 à $12 \mathrm{~mm}$ (7 à $24 \mathrm{~mm})$. Il pèse alors 28 à $37 \mathrm{dg}(8$ à $127 \mathrm{dg})$.

La présence d'un corps jaune modifie peu la longueur de la glande qui mesure alors le plus souvent 26 à $31 \mathrm{~mm}(17$ à $43 \mathrm{~mm})$. Elle a une influence plus nette sur la hauteur et l'épaisseur qui atteignent respectivement 23 à $24 \mathrm{~mm}(11$ à $30 \mathrm{~mm})$ et 15 à $16 \mathrm{~mm}$ (9 à $28 \mathrm{~mm})$. Dans les mêmes conditions physiologiques, le poids moyen de la glande va de 48 à $57 \mathrm{dg}$ (18 à $107 \mathrm{dg})$.

\section{I.C.) Variations}

CHEZ LA FEMELLE IMPUBERE, l'ovaire est notablement plus petit et moins lourd que chez la femelle adulte. Ses faces sont lisses jusqu’à la période qui précède de quelques semaines la première ovulation. 
AU COURS DU CYCLE OESTRAL l'ovaire subit des modifications périodiques qui, d'un di-oestrus à l'autre, se traduisent par l'apparition du relief provoqué par le follicule mûr, la formation du stigma, l'ovulation qui est précédée par une phase congestive intense, et la formation du corps progestatif dont l'involution est à peine ébauchée lorsque débute le cycle suivant.

PENDANT LA GESTATION, les corps gestatifs modifient profondément la conformation ovarienne. Celle-ci se caractérise en outre par la réapparition irrégulière des remaniements superficiels qui caractérisent la phase folliculaire du cycle oestral, qui peuvent aboutir, vers le $4 \mathrm{e}$, le $6^{\mathrm{e}}$ et le $7^{\mathrm{e}}$ mois, à des ovulations accompagnées des manifestations extérieures qui caractérisent les chaleurs.

CHEZ LA VACHE AGEE, la période active de la vie génitale est terminée. L'ovaire reste au repos. Il devient petit, scléreux et prend une coloration grisâtre.

\section{MOYENS DE FIXITE}

Primitivement placés en région sous-lombaire, au côté interne du mésonephros, les ovaires de Bos indicus subissent, comme ceux de Bos taurus, une MIGRATION PENDANT LA VIE FOETALE. A la naissance, ils sont situés à l'entrée du bassin et maintenus en place par un dispositif séro-ligamenteux complexe.

Le Méso de suspension du tractus génital femelle est le LIGAMENT LARGE. Dans sa portion la plus craniale, il est subdivisé ventralement en deux lames inégales, le MESOVARIUM, médial, qui s'insère sur l'ovaire, et le MESOSALPINX, latéral, qui soutient l'oviducte. Ces deux formations limitent une loge ou BOURSE OVARIQUE dans laquelle sont placés l'ovaire et le pavillon de l'oviducte. La lame conjonctive inter-séreuse des mésos juxta-ovariens se condense en outre localement pour former une série de LIGAMENTS qui unissent l'ovaire au reste du tractus génital et à la région sous-lombaire.

\section{II.A.) Le mésovarium}

Le mésovarium (Mesovarium) correspond à lá portion craniale du ligament large. Comme ce dernier, il se présente sous l'aspect d'un méso épais.

Son BORD DORSAL est fixe. Il s'insère à l'entrée du bassin sur les portions latérales de la région sous lombaire.

Son BORD VENTRAL s'insère sur l'ovaire.

Son BORD CRANIAL, oblique ventro-caudalement et légèrement médialement, est épais.

Son BORD CAUDAL est virtuel. Il se confond avec le ligament large.

La FACE INTERNE du mésovarium est lisse, tandis que la FACE EXTERNE présente dans sa portion ventrale un méso secondaire, le MESOSALPINX qui porte l'oviducte et contribue à la formation de la bourse ovarique.

La longue portion du mésovarium qui s'étend de la région sous-lombaire à l'insertion du mésosalpinx a reçu le nom de MESOVARIUM PROXIMAL (Mesovarium proximale) tandis que sa courte portion qui s'étend de la ligne d'insertion du mésosalpinx à l'ovaire est appelée MESOVARIUM DISTAL (Mesovarium distale).

\section{II.B.) Le mésosalpinx}

Le MESOSALPINX (Mesosalpinx) se détache de la portion ventrale de la face externe du mésovarium. Plus long que le mésovarium distal, il délimite une cavité à concavité dorsale en raison du report de son bord libre vers la face médiale du ligament large.

Son BORD DORSAL ou fixe décrit une courbe à concavité ventrale sur les parties déclives de la face externe du mésovarium. 
Son BORD LIBRE, reporté sur la face interne du dispositif séro-ligamenteux, est rectiligne et horizontal. Il limite ventralement l'orifice de la bourse ovarique.

La lame séro-conjonctive qui s'étend entre les deux bords forme les parois latérale et médioventrale de la bourse. Sa face profonde est concave, sa face superficielle convexe. Dans ses parties les plus déclives se trouve l'oviducte.

\section{II.C.) La bourse ovarique}

La BOURSE OVARIQUE (Bursa ovarica) est une cavité limitée par deux séreuses inégales. L'une très courte et médiale (le mésovarium distal) suspend par son bord libre la glande. L'autre très longue et latérale (le mésosalpinx) se replie médialement et porte l'oviducte.

La PAROI LATERALE de la bourse ovarique est donc entièrement constituée par le mésosalpinx.

Sa PAROI MEDIALE comprend deux éléments, l'un dorsal, le mésovarium distal qui porte l'ovaire et l'autre ventral qui correspond à la portion réfléchie du mésosalpinx.

L'ORIFICE DE LA BOURSE OVARIQUE est médial. Il se présente sous l'aspect d'une fente cranio-caudale plus ou moins étroite, comprise entre le bord libre de l'ovaire et celui du mésosalpinx. Sous l'action de fibres musculaires lisses, particulièrement abondantes dans les mésos qui le limitent, cet orifice peut se refermer complètement et isoler, au moment de l'ovulation, l'ovaire et le pavillon de l'oviducte de la cavité abdomino-pelvienne. Cette particularité explique l'extrême rareté sinon l'inexistence des gestations extra-utérines abdominales et pelviennes chez le zébu.

La CAVITE DE LA BOURSE OVARIQUE est réduite, presque virtuelle, en raison de son aplatissement médio-latéral. Son plafond se présente sous l'aspect d'un sinus étroit compris entre le mésovarium distal et la portion proximale du mésosalpinx. Son fond est concave cranio-caudalement. Ses extrémités en culs-de-sac étroits s'étendent, en avant, jusqu'au bord rostral du ligament large et, en arrière, jusqu'au voisinage de l'extrémité craniale de la corne utérine. Le pavillon de l'oviducte est situé dans le cul-de-sac cranial.

\section{II.D.) Les ligaments de l'ovaire}

Les ligaments qui fixent l'ovaire au reste du tractus génital et à la région sous lombaire sont: le ligament propre de l'ovaire, le ligament tubo-ovarique et le ligament suspenseur de l'ovaire.

\section{II.D.1.) LE LIGAMENT PROPRE DE L'OVAIRE}

Le LIGAMENT PROPRE DE L'OVAIRE (Ligamentum ovarii proprium) ou ligament utéro-ovarien unit la zone caudo-latérale de la glande à l'extrémité craniale de la corne utérine. Il correspond à un épaississement conjonctif, riche en fibres élastiques et en fibres musculaires lisses, de la portion caudale du bord libre du mésovarium distal.

\section{II.D.2.) LE LIGAMENT TUBO-OVARIQUE}

Le LIGAMENT TUBO-OVARIQUE ou frange ovarienne (Fimbria ovarica) correspond à l'adhérence de l'une des franges du pavillon de l'oviducte au pôle cranial de l'ovaire. L'union s'effectue par un renforcement du conjonctif sous-séreux de la face excentrique de la frange tubaire.

\section{II.D.3.) LE LIGAMENT SUSPENSEUR DE L'OVAIRE}

Le LIGAMENT SUSPENSEUR DE L'OVAIRE (Ligamentum suspensorium ovarii) correspond au bord cranial du mésovarium. Il s'étend obliquement ventro-caudo-médialement de 
la région sous-lombaire sur laquelle il se fixe au niveau de l'apophyse transverse de la dernière ou de l'avant-dernière vertèbre lombaire, à la région dorso-craniale de l'ovaire. Peu développé chez le zébu, il correspond structuralement à une densification discrète du conjonctif compris entre les deux lames séreuses du ligament large.

\section{TOPOGRAPHIE ET RAPPORTS}

La situation de l'ovaire est déterminée par sa continuité avec le reste du tractus génital et par le dispositif séro-ligamenteux qui fixe l'ensemble. Or, les dimensions de ces organes varient à chaque gestation. Elles sont aussi fonction du nombre des veaux auxquels la femelle a donné naissance. La topographie et les rapports de l'ovaire varient donc selon qu'on les examine sur une génisse, une vache gestante ou une vache multipare.

\section{III.A.) Topographie et rapports des ovaires de la génisse}

Chez la GENISSE, le tractus génital est en situation pelvienne. Les ovaires sont placés à l'entrée du bassin, dans le plan horizontal qui passe par les tubercules dinsertion des muscles petits psoas, et à mi-distance entre le plan médian du corps et le col de l'ilium. Ils sont faciles à découvrir par exploration rectale.

Dans cette situation, ils sont environnés par les circonvolutions de la portion distale du jéjunum et répondent médialement à la jonction recto-colique lorsque cette portion du gros intestin est en état de réplétion. En outre et selon le volume des viscères en cause, l'ovaire droit peut entrer en rapport avec l'apex caecal et l'ovaire gauche avec le sac aveugle caudo-dorsal du rumen.

\section{III.B.) Topographie et rapport des ovaires de la vache gestante}

Chez la VACHE GESTANTE, l'utérus gravide migre ventro-cranialement sur la déclivité de la paroi abdominale. Il entraîne avec lui le ligament large qui, grâce à sa laxité, se distend progressivement pendant toute la durée de la gestation. Le déplacement le plus important est effectué par la portion moyenne de la corne gravide et par la partie correspondante du ligament large. Le mésovarium et le ligament suspenseur de l'ovaire subissent la traction exercée par le reste du tractus génital. Fls s'allongent et s'orientent ventro-cranialement en entraînant l'ovaire dans une migration gravidique qui peut atteindre 10 à $15 \mathrm{~cm}$ pour la gonade correspondant à la corne gestante et qui place la glande en situation franchement abdominale.

\section{III.C.) Topographie et rapports des ovaires de la vache multipare}

Après la mise bas, le tractus génital et son dispositif de suspension subissent une régression rapide mais ne reprennent jamais complètement leurs dimensions primitives. Le ligament large est ainsi, dans son ensemble, d'autant plus haut et plus long que la femelle a donné naissance à un plus grand nombre de veaux. Ce double accroissement est responsable de la MIGRATION POST-GRAVIDIQUE des ovaires.

L'ACCROISSEMENT DE LA HAUTEUR DU MESOVARIUM se traduit pour l'ovaire par une position de plus en plus déclive, après chaque gestation. Chez les vieilles vaches, l'ovaire se trouve ainsi fréquemment en voisinage avec un plan horizontal qui passe par le planoher du bassin.

L'AUGMENTATION DE LA LONGUEUR DU LIGAMENT LARGE provoque deux déplacements successifs et différents de la gonade.

Dans un premier temps, l'ovaire subit une migration craniale qui le porte de la portion moyenne de l'entrée du bassin à un plan vertical passant par la limite rostrale de l'insertion du mésovarium, qui se trouve habituellement située au niveau du dernier espace interlombaire. 


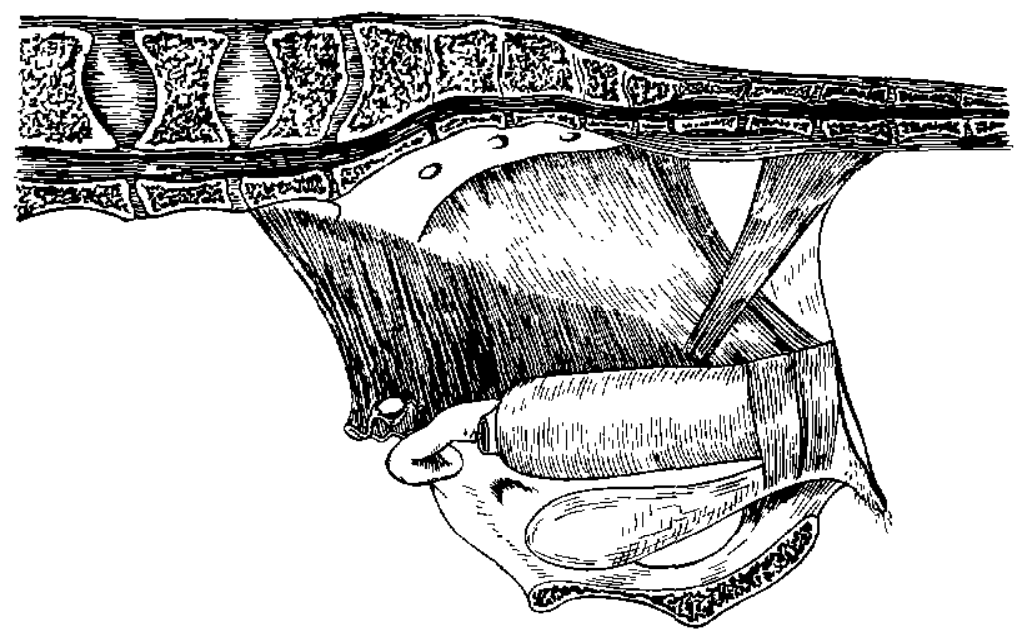

Fig. 2. - Topographie des organes génitaux de la femelle zébu primipare. (Vue demischérnatique latérale gauche, après ablation du ligament large, de la corne utérine, de l'oviducte et de l'ovaire gauches.)

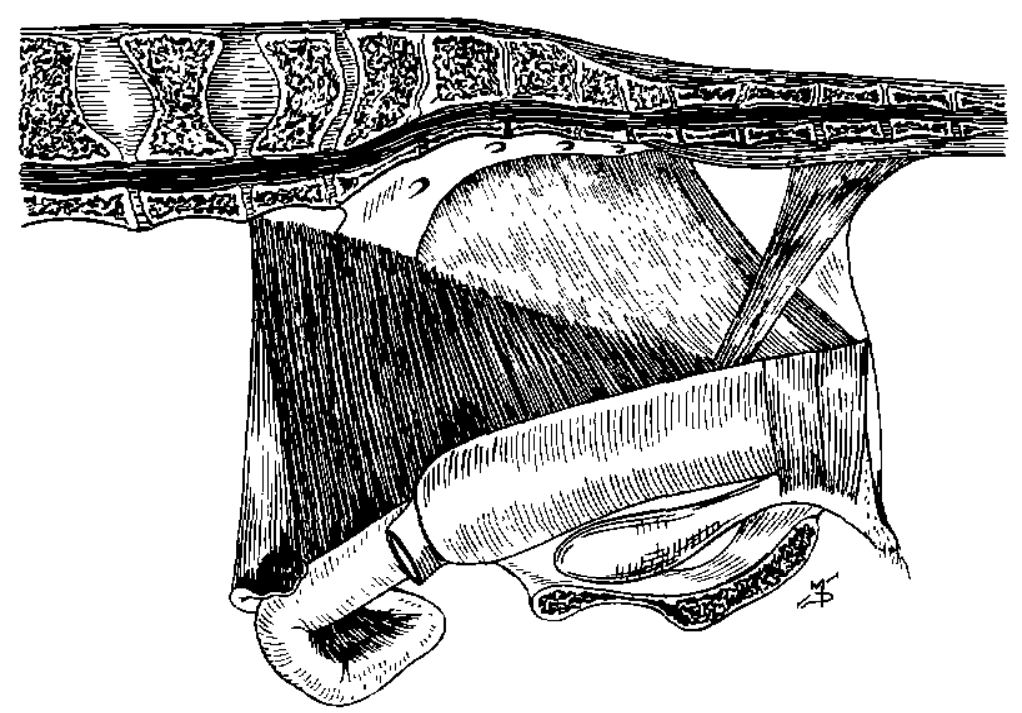

Fig. 3. - Topographie des organes génitaux de la femelle zébu multipare. (Vue demischématique latérale gauche après ablation du ligament large, de la corne utérine, de l'oviducte et de l'ovaire gauches.)

Cette limite atteinte, l'ovaire ne poursuit pas sa migration craniale, mais grâce à la mobilité que lui confère son dispositif suspenseur et aux orientations particulières des portions moyenne et rostrale des cornes utérines, la gonade et le mésovarium se replient médio-caudalement. L'orientation de l'ovaire et celle de la bourse ovarique se trouvent alors complètement inversées. L'extrémité tubaire de la glande devient caudale, tandis que son extrémité utérine devient craniale et que la position des faces se trouve aussi modifiée. Le mésovarium distal et l'orifice de la bourse ovarique prennent une orientation latérale et s'appliquent contre la face interne du ligament large, tandis que le mésosalpinx devient médial.

Au total, l'ovaire subit donc un premier déplacement ventro-cranial oblique qui l'amène au niveau vertical du dernier espace inter-lombaire, puis, secondairement, une simple migration ven- 
trale accompagnée d'une inversion de l'orientation. Ces particularités topographiques doivent être prises en considération lorsqu'une exploration rectale ou une ovariectomie en position quadrupédale sont effectuées sur un sujet âgé.

La migration gravidique de l'ovaire se produit aussi chez Bos taurus mais en raison de la disposition des cornes utérines de cette espèce, elle ne se traduit jamais par l'inversion des rapports de la glande. La migration chez les taurins parait en outre beaucoup plus importante et précoce que chez les zébus. Cette différence doit être rapportée aux faits que le premier vêlage s'effectue beaucoup plus tôt et que l'intervalle entre deux mises bas, est beaucoup plus court chez ceux-là que chez ceux-ci. Au même âge, la femelle des premiers ayant subi plus de gestations que celle des seconds, la migration gravidique de ses ovaires est beaucoup plus accusée.

Il est intéressant de remarquer que chez le zébu l'inversion de l'orientation ovarienne des vaches multipares ôte toute valeur aux termes d'orientations retenus par liste officielle des. N.A.V. pour désigner les faces de la glande (Facies lateralis et Facies medialis). Il serait souhaitable que, comme pour les pôles, des qualificatifs indépendants de l'orientation soient substitués aux précédents. Les termes FACE ANTRALE (Facies antralis) et FACE VISCERALE (Facies visceralis) qui font allusion aux rapports qu'entretiennent respectivement ces faces avec la cavité de la bourse ovarique et les viscères abdominaux pourraient être retenus et appliqués à toutes les espèces.

\section{VASCULARISATION, INNERVATION} et innervé.

Glande très active pendant toute la durée de la vie génitale, l'ovaire est richement irrigué

\section{IV.A.) L'artère ovarienne}

L'ARTERE OVARIENNE ( A ovarica dextra et sinistra) est une branche collatérale de l'aorte abdominale. Son origine est située à quelques centimètres de la terminaison de ce vaisseau, c'est-à-dire au niveau du disque intervertébral qui unit les corps des deux dernières vertèbres lombaires. L'artère ovarienne droite naît souvent un peu plus ventralement et caudalement que la gauche. Nous l'avons vue une fois se détacher de l'artère mésentérique caudale.

Après un court trajet transversal et sous péritonéal, l'artère ovarienne atteint le bord dorsal du mésovarium et s'engage dans ce méso. Elle se dirige ventro-caudalement, selon un trajet sinueux qui l'éloigne peu à peu du bord cranial de la lame séreuse. Avant d'atteindre le mésovarium distal, l'artère se divise en trois ou quatre branches qui pénètrent dans la glande par son hile et se distribuent au parenchyme ovarien.

Dans son trajet, l'artère ovarienne émet trois branches collatérales. De son bord cranial : les rameaux tubaires (Rami tubarii) cranial et moyen et de son bord caudal : le rameau utérin (Ramus uterinus) ou artère tubo-utérine des classiques français.

\section{IV.B.) La veine ovarienne}

La VEINE OVARIENNE (Vena ovarica dextra et sinistra) est volumineuse. Elle résulte de la convergence de veinules qui sortent de la glande par son hile. Satellite de l'artère correspondante, elle rejoint l'origine de la veine cave caudale. Ses affluents principaux sont une volumineuse veine marginale de l'utérus et le rameau veineux tubaire cranial.

\section{IV.C.) Lymphatiques de l'ovaire}

Les VAISSEAUX LYMPHATIQUES OVARIENS sont satellites des artères et des veines. Avec ceux de l'oviducte et des cornes utérines, ils rejoignent le groupe des GANGLIONS ILIAQUES MEDIAUX (Lymphodoni iliaci mediales). 


\section{IV.D.) Nerfs de l'ovaire}

Les NERFS DE L'OVAIRE appartiennent au système nerveux autonome. Ils sont représentés par de nombreux filets qui accompagnent les vaisseaux et procèdent du PLEXUS OVARIEN (Plexus ovaricus) situé à l'origine des artères ovariennes.

\section{ANATOMIE MICROSCOPIQUE DES OVAIRES}

Structuralement, les ovaires se caractérisent par la présence d'organites dont l'évolution conditionne le fonctionnement de l'ensemble du tractus génital.

\section{TOPOGRAPHIE STRUCTURALE}

Limité par un épithélium superficiel, l'ovaire est constitué d'un stroma conjonctif qui sert de support aux vaisseaux, aux nerfs et aux organites ovariens.

\section{I.A.) L'épithélium superficiel de l'ovaire}

L'EPITHELIUM SUPERFICIEL de l'ovaire (Epithelium superficiale), encore appelé épithélium germinatif, est formé d'une couche simple de cellules cubiques ou cylindriques qui peuvent prendre une disposition stratifiée dans les dépressions qui marquent la surface de la glande ou au niveau de l'étroite zone de transition qui le sépare de la séreuse du mésovarium distal (Margo limitans peritonei).

L'épithélium superficiel ne tapisse pas toute la surface de l'ovaire. Il est gênéralement absent du bord dorsal sur lequel se fixe le mésovarium distal et d'une portion plus ou moins importante de la face viscérale qui est recouverte par la lame séreuse interne du méso. Au niveau de cette ZONE DE RECOUVREMENT PERITONEAL, le conjonctif sous-séreux se densifie et est envahi par un réseau dense de gros vaisseaux, si bien que l'ovulation est impossible dans cette région. Les follicules en voie de maturation qui y migrent ne peuvent atteindre le stade de la déhiscence. Ils dégénèrent.

L'importance de la zone de recouvrement péritonéal varie avec les sujets. Souvent limitée à une faible portion du pôle utérin et de la face viscérale de la gonade, cette zone peut aussi prendre une telle extension que l'épithélium superficiel n'existe plus que sur la face antrale, le bord ventral et l'extrémité tubaire de la glande. Parfois enfin, la zone de recouvrement envahit le bord ventral de l'ovaire et limite ainsi l'étendue de la zone d'ovulation à la face antrale, à une partie des extrémités et au bord dorsal qui se trouve alors libéré de l'insertion mésovarienne.

\section{I.B.) Le stroma ovarien}

Le STROMA OVARIEN (Stroma ovarii) est un tissu conjonctif cellulaire. Au contact de l'épithélium superficiel, il s'oriente parallèlement à la surface en une mince COUCHE CONJONCTIVE SOUS EPITHELIALE. Dans le reste de son étendue, il est formé de tissu conjonctif d'aspect tourbillonnaire et se subdivise en une zone parenchymateuse et une zone vasculaire.

\section{I.B.1.) LA ZONE PARENCHYMATEUSE}

La ZONE PARENCHYMATEUSE (Zona parenchymatosa) occupe la plus grande partie de l'ovaire. Elle comprend une REGION CORTICALE (Cortex ovarii), superficielle, dans laquelle se localisent les organites les plus jeunes et une REGION MEDULLAIRE (Medulla ovarii), profonde, dans laquelle on observe des organites en cours d'évolution et parfois, au contact de la zone vasculaire, des vestiges du corps de Wolff qui forment le Rete ovarii. 

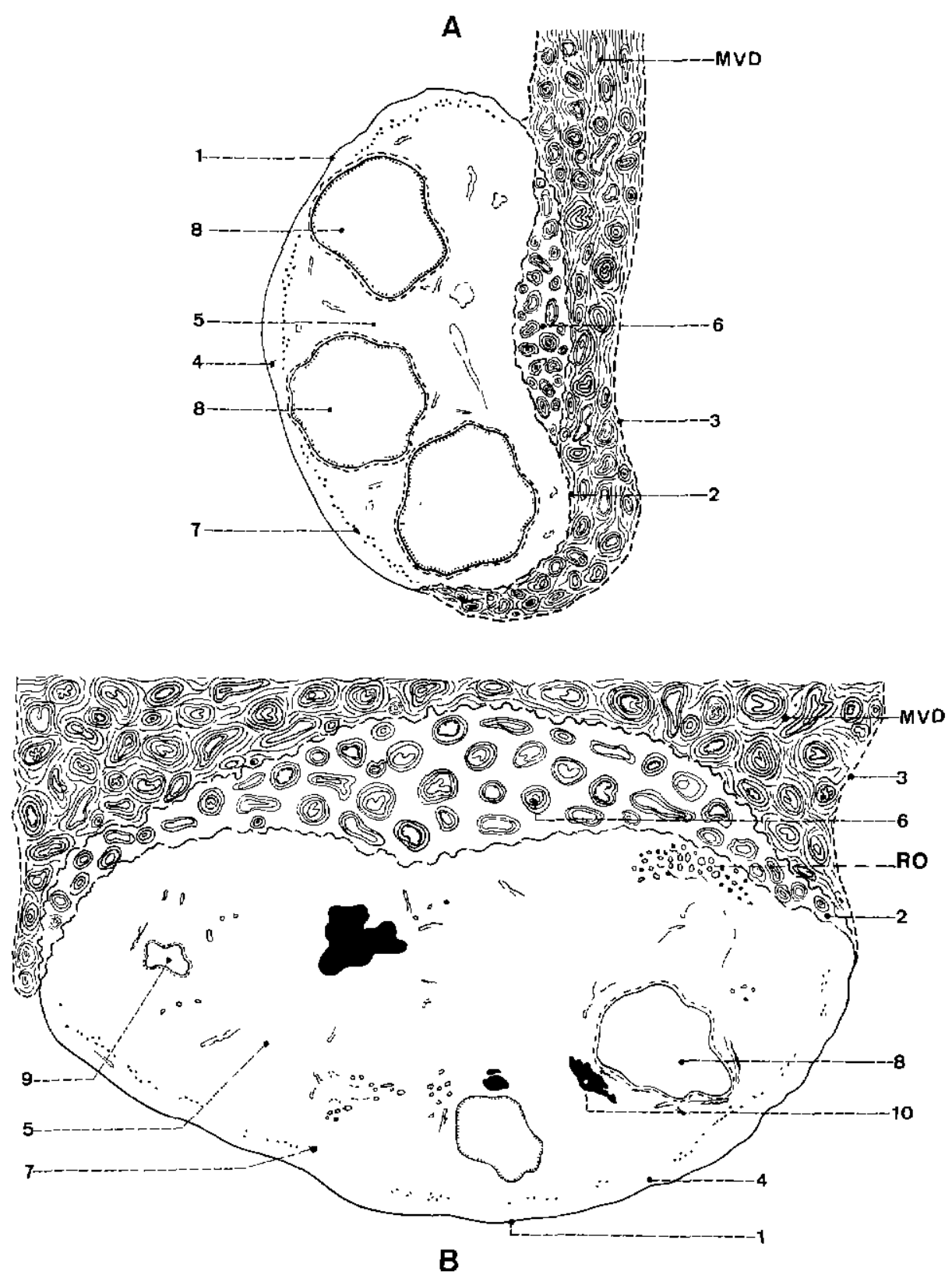

Fig. 4. - Histologie topographique de l'ovaire de la femelle du zébu. (Représentation demi-schématique.)

A) Coupe transversale d'un ovaire à recouvrement péritonéal médio-ventral.

B) Coupe longitudinale d'un ovaire à recouvrement péritonéal dorso-médial.

Légendes communes aux deux coupes: 1. Epithélium superficiel de l'ovaire; 2. Zone đe recouvrement péritonéal; 3. Lame séreuse épithéliale du mésovarium distal; 4. Couche conjonctive sous-épithéliale; 5 . Zone parenchymateuse; 6 . Zone vasculaire; 7. Follicules primaires; 8. Follicules cavitaires; 9. Follicules involutifs; 10. Corps blanes; M.V.D. Mésovarjum dista]; R.O. Rete ovarii.

\section{I.B.2.) LA ZONE VASCULAIRE}

La ZONE VASCULAIRE (Zona vasculosa) d'importance variable se situe au contact du hile et de la zone de recouvrement péritonéal. Elle est caractérisée par la présence de gros vaisseaux qui lui donnent un aspect spongieux. 


\section{I.C.) Vaisseaux et nerfs intra-ovariens}

\section{I.C.1.) SYSTEME ARTERIEL OVARIEN}

Les artères ovariennes proviennent d'un PLEXUS ARTERIOLAIRE localisé dans la zone vasculaire. Plus ou moins flexueux ou hélicoïdaux, ces vaisseaux portent le nom d'ARTERES HELICINES (Arteriae helicinae) et se distribuent aux thèques folliculaires et aux corps gestatifs et progestatifs.

\section{I.C.2.) SYSTEME VEINEUX OVARIEN}

Les veinules, issues des organites se rassemblent près du hile en un PLEXUS VEINEUX qui donne naissance aux racines de la veine ovarienne.

\section{I.C.3.) SYSTEME LYMPHATIQUE OVARIEN}

Les lymphatiques de l'ovaire sont nombreux autour des organites. Ils convergent vers le hile où l'on peut observer des vaisseaux lymphatiques valvulés.

\section{I.C.4.) NERFS OVARIENS}

Les NERFS OVARIENS appartiennent au système nerveux autonome. Ils se distribuent dans la zone parenchymateuse, autour des organites (composante sensitive) et dans la paroi des vaisseaux (composante vaso-motrice).

\section{LES ORGANITES DE L'OVAIRE}

Outre les terminaisons vasculaires et nerveuses, le stroma ovarien contient des organites issus des cordons germinatifs embryonnaires : les follicules évolutifs et involutifs et les corps progestatifs et gestatifs. Des images ovariennes particulières peuvent en outre être observées chez le zébu. Elles caractérisent les périodes de repos sexuel ou anoestrus.

\section{II.A.) Les follicules évolutifs}

Les FOLLICULES EVOLUTIFS, doués de la fonction gamétogène, se divisent morphologiquement en deux groupes : les follicules ovariens pleins et les follicules ovariens cavitaires.

\section{I.A.1.) LES FOLLICULES OVARIENS PLEINS}

Les follicules ovariens pleins sont localisés dans la région corticale de la zone parenchymateuse. Sous l'épithélium ovarien et sa lame conjonctive sous-épithéliale, ils se répartissent en une couche le plus souvent uni ou bi-stratifiée, continue ou interrompue par l'interférence d'organites plus volumineux.

Chez la jeune femelle, ils sont sensiblement moins nombreux chez Bos indicus que chez Bos taurus. Leur nombre diminue rapidement avec l'âge; une portion involue et l'autre se transforme en follicules cavitaires. Vers la $10^{\mathrm{e}}$ ou la $11^{\mathrm{e}}$ année, ils deviennent très rares.

Selon leur morphologie, on distingue deux types de follicules pleins.

Les FOLLICULES PRIMORDIAUX (Folliculi ovarici primordiales) sont constitués par un OVOCY'TE (Oocytus) sphérique, entouré d'une couche simple de CELLULES FOLLICULEUSES aplaties, puis cubiques et d'une VITREE (Membrana basalis folliculi). Cette dernière sépare les éléments épithéliaux (ovocytes et cellules folliculeuses) du stroma ovarien d'origine mésenchymateuse.

Dans les FOLLICULES PRIMAIRES (Folliculi ovarici primarii), les cellules folliculeuses se sont multipliées. Elles se disposent en plusieurs couches autour de l'ovocyte qu'elles entourent d'une masse arrondie ou ovalaire. 


\section{II.A.2.) LES FOLLICULES OVARIENS CAVITAIRES}

Les FOLLICULES OVARIENS CAVITAIRES ou ANTRAUX sont caractérisés par la présence d'une cavité folliculaire et par la différenciation progressive à la périphérie de la vitrée, d'une enveloppe conjonctive : la thèque folliculaire.

\section{II.A.2.a) La cavité folliculaire}

La CAVITE FOLLICULAIRE (Antrum folliculi) résulte de la sécrétion par les cellules folliculeuses d'un liquide intercellulaire. Les lacunes produites par cette sécrétion confluent et forment une cavité remplie par un liquide épais et coagulable par les fixateurs habituels: le LIQUIDE FOLLICULAIRE (Liquor folliculi).

La cavité est d'abord petite et en forme de croissant. Elle sépare la masse des cellules folliculeuses en deux portions. L'une, convexe qui contient l'ovaire, est le MASSIF GERMINAL (Cumulus oophorus). L'autre, concave et adossée à la vitrée, est la Granulosa.

Peu à peu, la cavité folliculaire grandit. Le follicule augmente de volume et devient sphérique. Les cellules folliculeuses sont alors refoulées contre la vitrée par la liqueur folliculaire. Au niveau de la Granulosa elles forment une couche de plus en plus mince, la COUCHE GRANU. LEUSE (Stratum granulosum) qui finit par être réduite à 4 ou 5 assises cellulaires. Autour de l'ovocyte, refoulé lui aussi vers la périphérie, les cellules folliculeuses restent groupées au niveau du MASSIF GERMINAL et forment un relief dans la cavité folliculaire.

Autour de l'ovule, les cellules folliculeuses déposent une formation exoplastique dense, la MEMBRANE PELLUCIDE ou ZONE PELLUCIDE (Zona pellucida) à la périphérie de laquelle les cellules s'alignent radiairement et constituent la COURONNE RADIEE (Corona radiata).

\section{II.A.2.b) La thèque folliculaire}

Autour de la vitrée, les cellules conjonctives du stroma ovarien s'organisent et forment la THEQUE FOLLICULAIRE (Theca folliculi).

Les cellules conjonctives les plus proches de la vitrée deviennent polyédriques et portent alors le nom de CELLULES THECALES (Thecocytus). Celles-ci forment une couche continue de 4 à 5 assises cellulaires : la THEQUE INTERNE (Theca interna) ou COUCHE INTERNE DE LA THEQUE FOLLICULAIRE (Tunica interna thecae folliculae).

Autour de cette couche thécale interne, le stroma ovarien s'organise en formations lamelleuses concentriques dans lesquelles se développe un réseau vasculaire. Cette couche qui croît en même temps que le follicule, correspond à la THEQUE EXTERNE (Theca externa) ou COUCHE EXTERNE DE LA THEQUE FOLLICULAIRE (Tunica externa thecae folliculae).

\section{II.A.2.c) Classification des follicules cavitaires}

Selon leur topographie, leur morphologie et le stade de leur évolution, les follicules cavitaires se divisent en FOLLICULES EN CROISSANCE (Folliculi ovarici crescentes) distingués euxmêmes en petits, moyens et grands et en FOLLICULES MURS (Folliculi ovarici vesiculosi).

- Les FOLLICULES CAVITAIRES PETITS ET MOYENS proviennent des follicules primaires. Au proestrus de chaque cycle, un contingent de ces derniers subit la transformation cavitaire. Au fur et à mesure qu'ils grossissent, ils migrent vers la portion médullaire de la zone parenchymateuse. Puis, ayant atteint le stade follicule cavitaire petit ou moyen, ils cessent leur évolution et constituent un stock de réserve qui s'épuise périodiquement par maturation ou involution et se renouvelle à partir de la masse des follicules primaires de la zone corticale. 
- Les GRANDS FOLlicules CAVITAIRES et les Follicules MURS ne peuvent être observés qu'au moment du pro-oestrus. Sous l'action de l'hormone gonadotrope F.S.H. qui est aussi responsable de la transformation des follicules primaires en petits et moyens follicules cavitaires, un ou plusieurs de ces derniers subissent au cours de cette phase du cycle oestral une évolution qui amène au moins l'un d'entre eux aux stades gros follicule cavitaire et follicule mûr.

Localisés d'abord dans la portion médullaire de la zone parenchymateuse, le follicule cavitaire se rapproche de la surface de l'ovaire en même temps qu'il grossit. Au stade follicule mûr, il est accolé à l'épithélium superficiel et forme un relief à la surface de la glande. Au niveau de la zone de contact, ou TACHE TRANSLUCIDE (Macula pellucida), la thèque externe devient très mince et presque avasculaire tandis que la thèque interne diminue d'épaisseur, que la couche granuleuse se réduit à 2 ou 3 assises cellulaires et que le massif germinal se dissocie par apparition de lacunes irrégulières remplies de liqueur folliculaire. Cette dernière s'accumule aussi dans la cavité folliculaire et par sa pression tend les parois du follicule. En dehors de la tache translucide, les cellules de la thèque interne grossissent. Leur cytoplasme s'enrichit d'inclusions lipidiques qui correspondent vraisemblablement à une sécrétion d'œstrogènes. La thèque externe se vascularise fortement. Elle délègue vers la thèque interne un réseau dense de vaisseaux capillaires.

Généralement 1 à 5 follicules cavitaires commencent le processus de la croissance. Mais ce nombre se réduit toujours au cours de l'évolution. Chez Bos indicus en effet, à chaque cycle, un seul follicule arrive habituellement au stade follicule mûr. Les autres dégénèrent plus ou moins précocement, si bien qu'il n'est pas rare d'observer en même temps qu'un follicule mûr deux ou trois grands follicules cavitaires frappés de nets signes d'involution. Cette particularité est en accord avec les observations des zootechniciens, selon lesquelles la gémellarité est très rare chez le Zébu. Lors d'une série de prélèvements effectués sur 552 femelles pleines à l'abattoir de Dakar, nous n'en avons observé que deux cas, soit 3,62 p. 1000.

- Le FOLLICULE DEHISCENT caractérise l'œstrus. C'est un follicule mûr dont la paroi vient de se rompre pour permettre la libération de l'ovule.

Le remaniements structuraux qui caractérisent l'ovulation sont induits par l'hormone gonadotrope L.H. Pendant qu'au contact de l'épithélium superficiel les parois du follicule s'amincissent, l'ovocyte entouré de la zone pellucide et de la couronne radiée se détache de la paroi et flotte dans la liqueur folliculaire. Une nécrose provoquée par blocage local de l'irrigation sanguine se produit ensuite au niveau de la tache translucide qui se rompt sous l'action de la pression intra-folliculaire et de la turgescence temporaire du parenchyme glandulaire. L'ovule entraîné par la liqueur folliculaire est projeté par l'orifice ou Stigma dans le pavillon de l'oviducte qui s'est accolé à l'ovaire.

A ces remaniements structuraux de l'œstrus correspondent des manifestations extérieures ou chaleurs dont la chronologie par rapport au moment de l'ovulation est intéressante à préciser.

Dans les conditions habituelles de l'élevage en région tropicale sèche, les chaleurs de la femelle zébu sont discrètes. Elles se produisent souvent la nuit.

Selon certains observateurs, elles peuvent même être totalement invisibles et correspondre alors à l'estrus silent heat ou CSTRUS A CHALEURS SILENCIEUSES défini par les anglosaxons comme un estrus ovarien normal totalement dépourvu de signes extérieurs.

Les chaleurs de la femelle zébu sont aussi souvent très courtes. Pour en faire une analyse précise, les zootechniciens ont coutume de les diviser en trois périodes. Au cours de la première période, la femelle attire le mâle mais ne l'accepte pas. Pendant la seconde, la femelle consent à la saillie. Au cours de la troisième période, elle refuse à nouveau le mâle mais continue à l'attirer.

La période totale d'attirance du mâle dure en moyenne de 13 à 21 heures (14 à 16 heures 
pour le zébu Gobra). Les valeurs extrêmes ont été signalées sur du bétail d'Uganda : 7,47 heures et 43,27 heures.

La période pendant laquelle la femelle accepte le mâle se situe à peu près au milieu de la période précédente. Elle dure le plus souvent de 4 à 6 heures (5 à 6 heures pour le zébu Gobra). Ses valeurs extrêmes ont aussi été observées sur le bétail d'Uganda : 12 minutes et 36 heures.

Par rapport à ces manifestations extérieures de l'œstrus, l'ovulation s'effectuerait tardivement. Après la fin de la troisième période, vers la 14e heure qui suit la disparition des chaleurs, selon des travaux réalisés sur la race Hariana. Cette particularité justifie la pratique d'inséminations artificielles tardives.

Lorsqu'un follicule en croissance migre vers la zone de recouvrement péritonéal, il grossit mais, incapable d'entrer en contact avec l'épithélium superficiel, il involue avant d'atteindre le stade de la maturation.

Ce phénomène explique les CHALEURS ANOVULATOIRES qui ont été signalées chez le zébu. Comme chez la jument dont les gonades présentent aussi une importante et constante zone de recouvrement péritonéal, il pourrait être responsable aussi des chaleurs de durée anormalement longue.

- LES FOLLICULES A PLUSIEURS OVOCYTES, bien que rares, ne sont pas exceptionnels chez le zébu. Trois sujets sur 415 que nous avons étudiés par coupes sériées des glandes génitales ont présenté sur un seul ou sur les deux ovaires des follicules dans lesquels nous avons pu dénombrer 8 à 15 ovocytes par coupe, tous situés dans autant de massifs germinaux répartis en couronne autour de la cavité folliculaire.

\section{II.B.) Les follicules involutifs}

A chaque étape de la transformation des follicules évolutifs, une partie des organites dégénère et constitue la masse des FOLLICULES INVOLUTIFS (Folliculi atretici) perdus pour la fonction gamétogène mais capables d'acquérir, au moins pour certains d'entre eux, la fonction endocrine. Chez le zébu, ils se présentent sous quatre formes.

\section{II.B.1.) LES FOLLICULES DEGENERATIFS}

Les FOLLICULES DEGENERATIFS représentent la forme involutive des follicules primaires. Ils sont caractérisés par une histolyse rapide et totale de l'ovule et des cellules folliculeuses qui les constituent. Ils sont dépourvus de fonction hormonale et disparaissent sans laisser de trace. Peut-être, en raison de la rapidité de leur involution, on ne les observe que très exceptionnellement chez le zébu.

\section{II.B.2.) LES FOLLICULES ATRETIQUES}

Les FOLLICULES ATRETIQUES dérivent des follicules cavitaires petits et moyens. Ils sont particulièrement nombreux chez Bos indicus. Selon les modalités involutives dont ils sont l'objet, ils se présentent sous trois aspects principaux.

\section{II.B.2.a) Les follicules atrétiques de type I ou follicules atrétiques à dégénérescence épithéliale complète}

Le FOLLICULE ATRETIQUE DE TYPE I, classiquement décrit chez les mammifères, est caractérisé par une dégénérescence rapide des formations épithéliales (ovule et cellules folliculeuses) et la persistance plus ou moins longue des thèques conjonctives dont l'interne s'hypertrophie et acquiert la FONCTION CESTROGENE.

Au stade sécrétoire, ce follicule se présente sous l'aspect d'une formation sphérique ou ovoïde (les thèques) centrée sur une cavité qui correspond aux formations épithéliales dispa- 
rues. Cette cavité est limitée par la vitrée épaissie ou par une couche simple de cellules conjonctives aplaties qui remplacent la vitrée Iorsqu'elle aussi disparaît.

Plus tard, les thèques subissent la dégénérescence hyaline. Le follicule atrétique, comprimé par les organites voisins, se déforme. Sur les coupes, sa cavité centrale prend l'aspect d'un triangle, d'un croissant ou d'une étoile. Le follicule devient un corps blanc d'atrésie, toujours facilement identifiable grâce à la cavité qui persiste en son centre.

En fin d'évolution, le corps blanc est peu à peu envahi par les cellules conjonctives du stroma ovarien, puis il disparaît.

\section{II.B.2.b) Les follicules atrétiques de type II ou follicules atrétiques à ovocyte persistant}

Les follicules atrétiques de type II diffèrent du type précédent par la persistance plus ou moins longue de l'ovocyte ou de ses traces. La cavité centrale qui résulte de la disparition rapide des cellules folliculeuses contient un ovocyte plus ou moins altéré. Au stade du corps blanc d'atrésie on peut souvent observer, dans une cavité centrale réduite, la membrane pellucide épaissie et souvent festonnée.

\section{II.B.2.c) Les follicules atrétiques de type III ou follicules atrétiques à granulosa persistante}

La troisième forme des follicules atrétiques du zébu est caractérisée par la dégénérescence rapide de l'ovocyte et la persistance des cellules folliculeuses qui perdent leur pouvoir sécrétoire, se multiplient rapidement et remplissent entièrement la cavité folliculaire.

Au stade sécrétoire de la thèque, puis au stade corps blanc d'atrésie qui lui succède, ces organites sont caractérisés par la présence d'une masse centrale de cellules folliculeuses.

\section{II.B.3.) LES FOLLICULES KYSTOIDES}

Les FOLLICULES KYSTOIDES dérivent des follicules cavitaires moyens et grands. Ils sont très rares chez le zébu. Ils sont caractérisés par la dégénérescence de l'ovule et la persistance des cellules folliculeuses qui, contrairement à celles des follicules atrétiques de type III, conservent leur propriété sécrétoire et se multiplient modérément, de place en place, pour former de gros bourrelets qui forment des festons à la périphérie de l'Antrum rempli de liqueur folliculaire sous tension.

A la périphérie, la thèque interne se développe et acquiert la fonction œstrogène. La thèque externe s'épaissit mais ne forme jamais comme chez Bos taurus une coque à paroi épaisse qui transforme le follicule en véritable kyste.

La régression du follicule kystoïde du zébu est caractérisée par l'émission de travées conjonctives issues du stroma environnant. Ces dernières prolifèrent en direction de la cavité folliculaire et provoquent la dégénérescence des éléments sécrétoires de la thèque et de la granulosa. Des débris de feston folliculeux, d'aspect plus ou moins grumeleux et centrés sur une cavité irrégulière peuvent persister longtemps dans le parenchyme ovarien.

\section{II.B.4.) LES FOLLICULES LAMELLEUX}

Les FOLLICULES LAMELLEUX dérivent des follicules mûrs et des grands follicules en croissance qui ont migré vers la zone de recouvrement péritonéal. Ils sont aussi la forme habituelle d'involution des follicules cavitaires qui commencent leur évolution en même temps que le follicule libérateur d'ovule, mais n'atteignent pas le stade de la maturité. Ils dérivent donc des follicules mûrs ou des grands follicules cavitaires et ne se forment que très exceptionnellement à partir des follicules cavitaires moyens. 

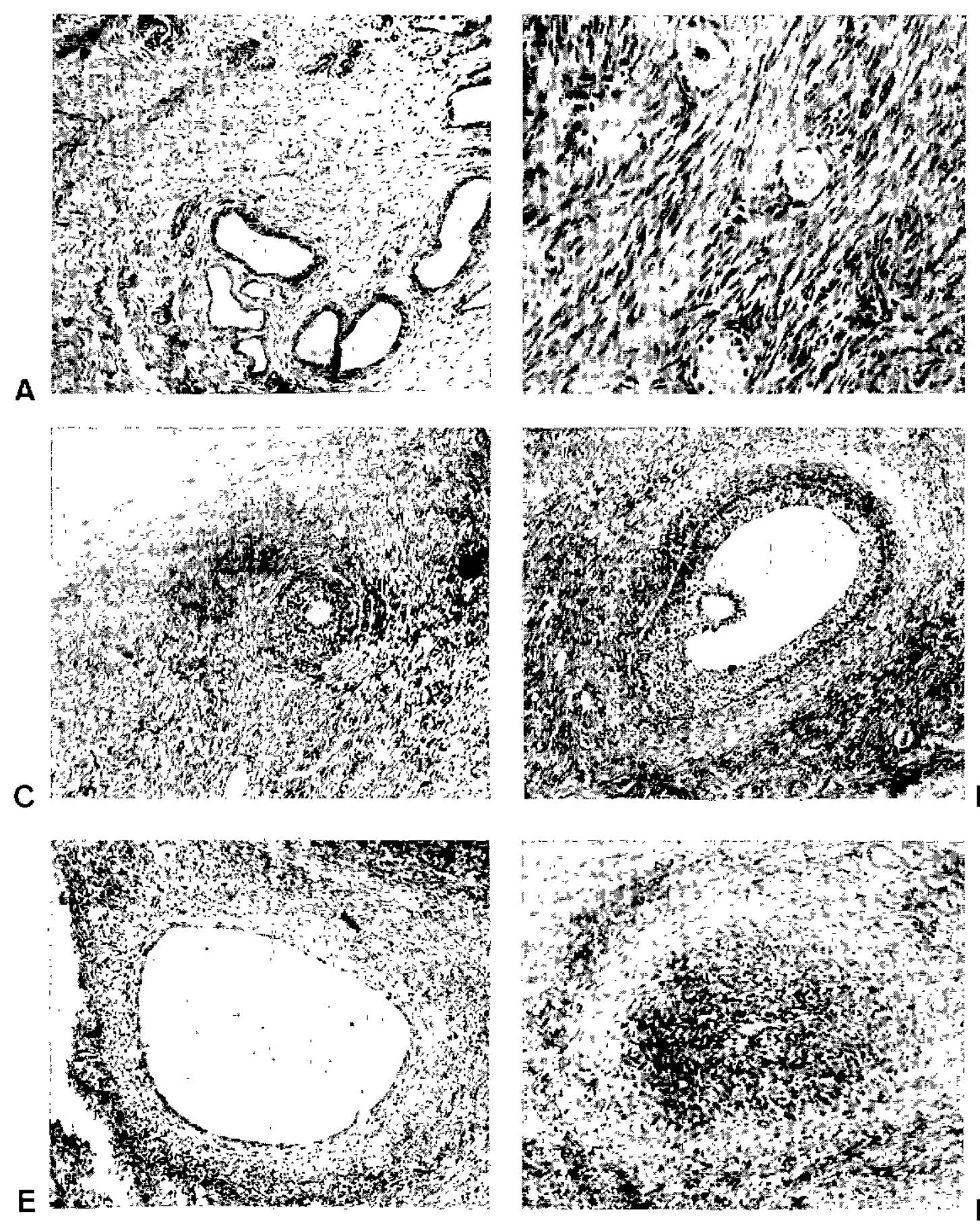

Fig. 5.1. - Structure de l'ovaire de la femelle zébu.

A) Zone vasculaire de l'ovaire $(\times 250)$.

B) Follicules ovariens primordiaux $(\times 400)$.

C) Follicule ovarien primaire $(\times 250)$.

D) Follicule ovarien en croissance $(X 250)$.

E) Follicule ovarien atrétique à dégénérescence épithéliale complète $(\times 250)$.

F) Follicule ovarien atrétique à granulose persistante $(\times 250)$. 
A
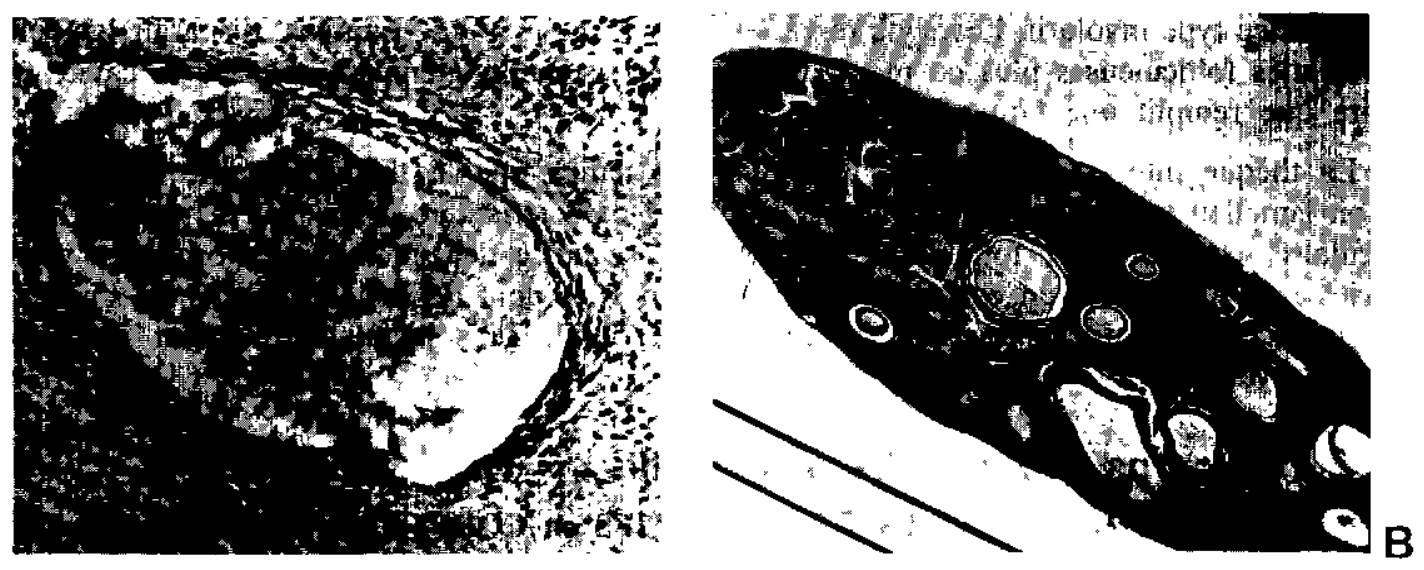

C
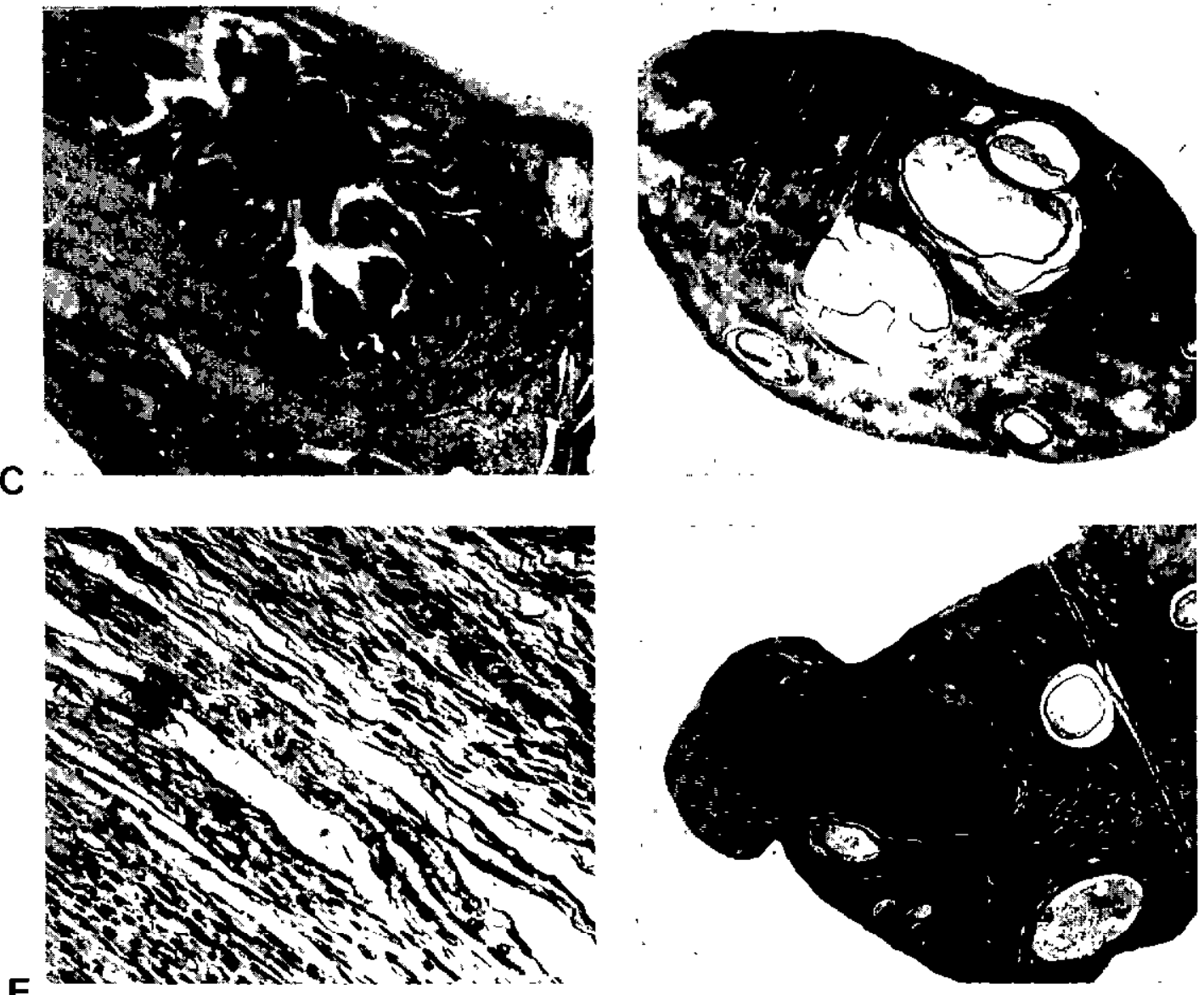

Fig. 5.2. - Structure de l'ovaire de la femelle zébu.

A) Follicule atrétique à ovocyte persistant ( $\times 250$ ).

B) Follicule kystoïde (à l'extrémité gauche de l'ovaire) (X 12).

C) Follicule kystoïde ( $\times 100$ ).

D) Follicules lamelleux $(\times 12)$.

E) Zone thécale d'un follicule lamelleux ( $\times$ 400),

F) Corps jaune cyclique extra- et intra-ovarien dit en a bouchon de champagne» $(X 12)$. 
Dans ce type involutif, l'ovocyte régresse rapidement mais la granulosa disparaît lentement. Des cellules folliculeuses plus ou moins désunies persistent longtemps autour de la cavité folliculaire que remplit une liqueur altérée.

La thèque interne régresse et subit en même temps que la thèque externe une transformation lamelleuse qui lui donne un aspect feuilleté caractéristique. Puis, peu à peu, la liqueur folliculaire se résorbe. Le follicule devient mou et se laisse déformer par les organites voisins. La vitrée se rétracte et un espace la sépare des thèques dont les lamelles tendent à s'isoler les unes des autres. Enfin l'ensemble subit une lyse rapide sans jamais être atteint de dégénérescence hyaline.

\section{II.C.) Les corps progestatifs et gestatifs}

Les CORPS PROGESTATIFS ET GESTATIFS ou CORPS JAUNES (Corpora lutea) se développent à partir des follicules déhiscents. Ils se transforment en glandes endocrines, d'activité courte (Corps progestatifs) ou prolongée si l'ovulation a été suivie de fécondation (Corps gestatifs).

\section{II.C.1.) LES CORPS PROGESTATIFS}

Les CORPS PROGESTATIFS ou CORPS JAUNES CYCLIQUES (Corpora lutea cyclica) se renouvellent à chaque cycle. Leur évolution est caractérisée par les phases suivantes : développement, état, involution et dégénérescence,

\section{II.C.1.a) Phase de développement}

$\mathrm{Au}$ début de la phase de développement, la cavité du follicule est remplie par un exsudat séro-fibrineux auquel la micro-hémorragie qui accompagne la déhiscence confère une coloration rougeâtre. A ce stade, l'organite est, pour cette raison, appelé CORPS ROUGE (Corpus hemorrhagicum).

Des cordons cellulaires issus de la couche granuleuse et de la thèque interne prolifèrent et envahissent selon trois modalités possibles le caillot séro-fibrineux qui déborde largement du STIGMA.

Dans la première modalité, la plus fréquente, les cordons cellulaires envahissent la portion intra-folliculaire du caillot dont la portion externe disparaît. Un CORPS JAUNE INTRAOVARIEN se constitue. Il refoule latéralement et dans la profondeur de la glande les organites et le stroma ovariens.

Dans la deuxième modalité, souvent observée, des cordons cellulaires envahissent la totalité du caillot. Il en résulte l'apparition d'un CORPS JAUNE EXTRA ET INTRA-OVARIEN qui, sur les coupes, affecte l'aspect « en bouchon de champagne » classiquement décrit chez Bos taurus.

Dans la troisième modalité, plus rare, mais non exceptionnelle, les cordons cellulaires ne s'organisent que dans la portion externe du caillot. Il en résulte l'apparition d'un CORPS JAUNE EXTRA-OVARIEN uni à l'ovaire par un pédicule plus ou moins grêle.

A la fin de la phase de développement, les cellules folliculeuses se transforment en cellules progestatives et les cellules thécales prennent la fonction œstrogène.

\section{II.C.1.b) Phase d'état}

Pendant la phase d'état, les cellules sécrétrices accumulent dans leur cytoplasme une substance lipidique riche en pigments caroténoïdes, la lutéine, qui les colore en jaune orangé. Le corps rouge se transforme en CORPS JAUNE (Corpus luteum) et les cellules deviennent des LUTEOCYTES (Luteocyti). 
A
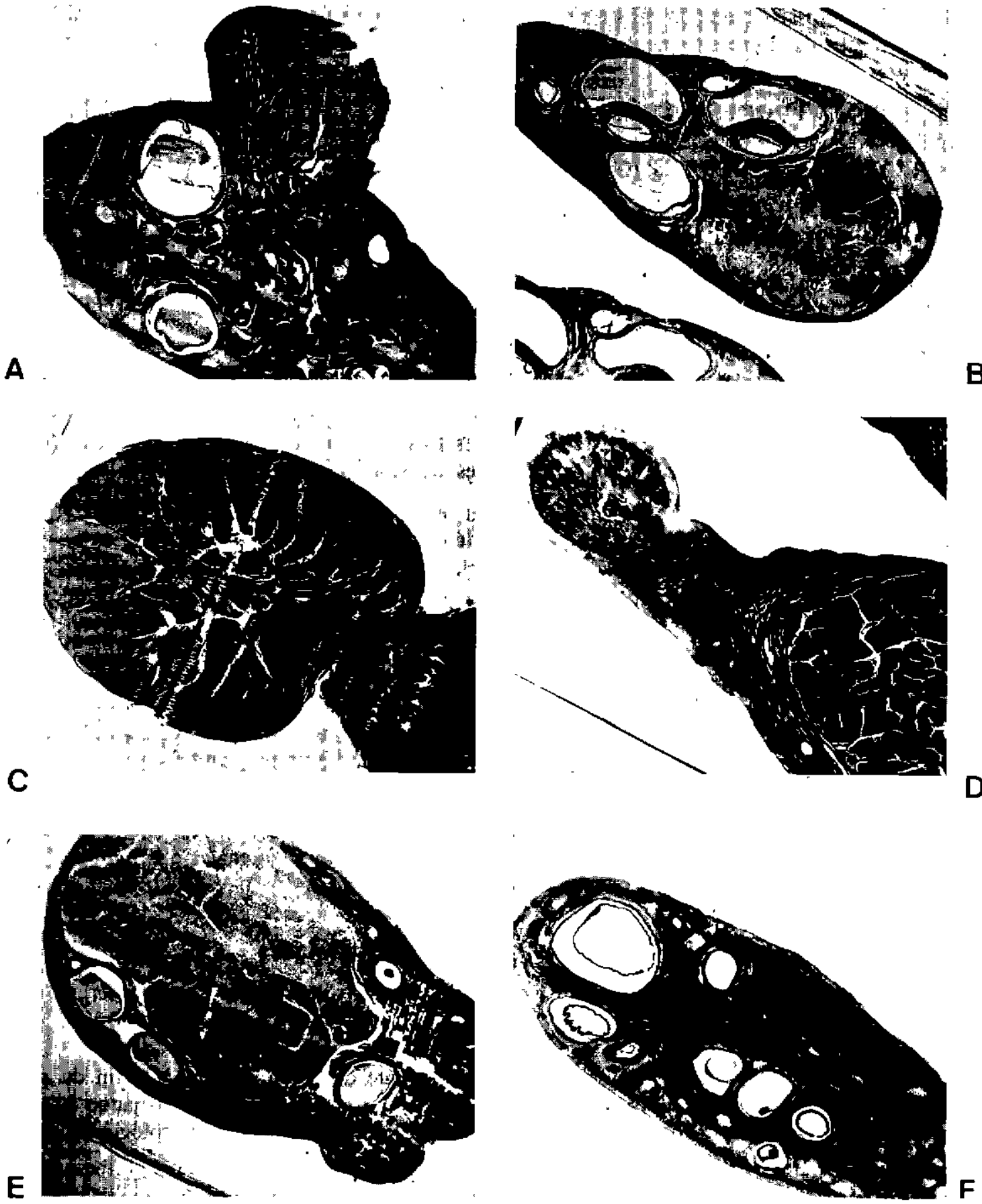

Fig. 5.3. - Structure de l'ovaire de la femelle zébu.

A) Corps jaune cyclique extra-ovarien $(\times 12)$.

B) Corps jaune cyclique intra-ovarien ( $X 12$ ).

C) Corps gestatif primaire extra-ovarien (au pôle ovarien corps blanc d'origine cyclique) $(\times 12)$.

D) Corps gestatif secondaire (en position intra-ovarienne) ( $\times$ 12). Le corps gestatif primaire est extra-ovarien.

E) et F) Corps gestatifs primaire, secondaire et tertiaire.

E) Ovaire droit : corps gestatif tertiaire (intra-ovarien) et corps gestatif secondaire (extraovarien) $(\times 12)$.

F) Ovaire gauche: corps gestatif primaire (à droite du grand follicule involutif de type lamelleux) (X 12). 
A faible grossissement, la masse du parenchyme glandulaire a un aspect homogène, mais un examen attentif, à grossissement plus fort, permet de distinguer deux types cellulaires regroupés en îlots.

Les ILOTS PROGESTATIFS, d'aspect réticulé, sont composés de grandes cellules chromophiles, ovalaires ou polygonales à angles arrondis et à noyaux clairs à chromatine en masse : les GRANULO-LUTEOCYTES (Granulosoluteocyti).

Les ILOTS THECAUX sont formés de cellules plus petites, polyédriques à angles saillants, à noyaux petits et foncés, à chromatine dense, les LUTEOCYTES THECAUX (Thecoluteocyti).

La thèque externe s'enrichit en artères hélicines et en veinules néo-formées. Elle délègue vers le parenchyme glandulaire de minces fusées vasculo-conjonctives qui assurent l'irrigation de l'ensemble.

\section{II.C.1.c) Phase d'involution}

Au début de la PHASE D'INVOLUTION, les très fines travées conjonctivo-vasculaires se densifient et divisent la glande en amas cellulaires anastomosés entre eux.

Puis, peu à peu, ces cloisons s'épaississent et les amas cellulaires s'isolent en îlots au sein desquels les cellules accroissent leur surcharge lutéinique. Cette dernière, bien que nette, n'atteint pas chez Bos indicus une intensité comparable à celle qu'on observe chez Bos taurus. Cette différence semble devoir être rapportée au régime alimentaire pauvre en caroténoïdes, que supporte le zébu pendant la plus grande partie de l'année.

\section{II.C.1.d) Phase de dégénérescence}

La PHASE DE DEgeNERESCENCE se caractérise par la disparition rapide des cellules glandulaires et des travées conjonctives qui se transforment, par imprégnation hyaline, en une masse parcourue de fibrilles collagènes. Cette transformation aboutit à la formation d'un CORPS BLANC (Corpus albicans) de même taille et de même forme que le corps jaune dont il dérive.

Puis le corps blanc, envahi par le stroma conjonctif, diminue lentement de volume et finit par disparaître.

\section{II.C.1.e) Chronologie de l'évolution des corps progestatifs}

La PHASE D'ORGANISATION suit immédiatement l'ovulation. Elle est très brève.

La PHASE D'ETAT qui correspond au post-œstrus lui succède.

La PHASE D'INVOLUTION est tardive. Elle débute de façon discrète à la fin du postœstrus et s'effectue lentement de sorte qu'au pro-œstrus du cycle suivant, le corps jaune du zébu ne présente pas comme celui des taurins des signes nets d'involution et qu'histologiquement le di-œstrus est difficile à distinguer du post-œstrus.

La PHASE DE DEGENERESCENCE est longue, si bien qu'au cours d'un cycle on peut observer plusieurs corps blancs à divers stades de régression qui correspondent à des cycles antérieurs. Au total, le corps blanc peut être encore visible 3 mois au moins après l'œstrus dont il est issu.

\section{II.C.2.) LES CORPS GESTATIFS}

Les CORPS GESTATIFS ou CORPS JAUNES DE GESTATION (Corpora lutea graviditates) sont des corps jaunes dont la phase d'activité est prolongée pendant tout ou partie de la gestation.

Au cours des PHASES DE DEVELOPPEMENT ET D'ETAT, ils sont structuralement 
identiques aux corps progestatifs. Morphologiquement, ils en diffèrent par un volume souvent plus grand et par l'importance du réseau vasculaire qui les entoure.

Leur PHASE D'INVOLUTION est très longue. Les cloisons conjonctives qui la caractérisent atteignent une grande épaisseur et peuvent, sur une coupe, occuper une surface plus importante que les îlots cellulaires en surcharge lutéinique.

La PHASE DE DEGENERESCENCE se traduit exceptionnellement par une imprégnation hyaline massive. Le plus souvent, la hyalinisation reste discrète et n'atteint que la périphérie et les cloisons conjonctives les plus épaisses du corps gestatif. Ce dernier est alors résorbé par des fusées cellulaires issues du stroma ovarien qui envahissent et morcellent l'organite en laissant longtemps persister entre elles de minuscules îlots glandulaires et de volumineux vaisseaux à leur périphérie.

Il est intéressant de remarquer que ce mode particulier de dégénérescence semble lié à l'état de gestation et non à la prolongation de la période d'activité du corps jaune. Le dernier corps jaune cyclique dont l'involution était à peine ébauchée lors de l'œstrus de fécondation se comporte, en effet, comme un corps gestatif pendant sa phase de dégénérescence.

FONCTIONNELLEMENT, les corps gestatifs du zébu présentent la remarquable particularité de n'être fréquemment actifs que pendant une partie seulement de la gestation. Alors que chez Bos taurus le corps jaune de fécondation persiste jusqu'au part, celui de Bos indicus peut être remplacé ou supplémenté par des CORPS JAUNES ACCESSOIRES (Corpora lutea graviditates accessorii) ou encore disparaître.

Le REMPLACEMENT DU CORPS GESTATIF PRIMAIRE s'effectue à une date variable à partir du $4^{e}$ mois de la gestation.

Le CORPS GESTATIF SECONDAIRE qui lui succède peut résulter de la métaplasie glandulaire d'un follicule déhiscent. La présence d'une zone d'adhérence du corps jaune à l'épithélium ovarien et l'existence d'œstrus survenant entre le $4^{\mathrm{e}}$ et le $7^{\mathrm{e}}$ mois de la gestation le confirment. Le plus souvent, cependant, le corps gestatif secondaire dérive d'un follicule qui n'atteint pas la déhiscence. Il se développe plus fréquemment sur l'ovaire qui ne porte pas le corps gestatif primaire que sur l'autre.

De taille souvent plus petite que son prédécesseur, le corps gestatif secondaire subit la même évolution que lui. Ce dernier reste longtemps visible en raison de la longueur de sa phase d'involution.

A partir du $6^{e}$ mois, le corps gestatif secondaire peut à son tour être remplacé par un CORPS GESTATIF TERTIAIRE. A côté du corps jaune en activité, on en observe alors deux autres à des phases différentes de leur involution. Selon nos observations, 42 p. 100 des femelles examinées entre le $6^{\mathrm{e}}$ mois et le part subissent ce double remplacement du corps jaune de fécondation. Sur certains sujets de ce groupe, la présence de gros follicules cavitaires en phase de maturation laisse supposer la possibilité du développement d'une $4^{\mathrm{e}}$ génération de corps gestatifs.

La SUPPLEMENTATION DU CORPS GESTATIF peut se produire à n'importe quel moment de la gestation. Sa fonction est de suppléer à l'insuffisance fonctionnelle d'un corps gestatif d'une génération quelconque. Le CORPS GESTATIF DE SUPPLEMENTATION dérive d'un follicule cavitaire de taille moyenne. Il est facilement identifiable en raison de sa taille réduite et de sa situation profonde dans la zone médullaire du stroma ovarien. Son développement et son évolution sont comparables à ceux des corps gestatifs vrais. Ils diffèrent cependant de ces derniers par une ségrégation particulière des cellules de la couche granuleuse et de la thèque interne. Ces cellules se multiplient sur place si bien que les granulo-lutéocytes occupent dans le corps jaune une position centrale qui correspond à l'ancienne cavité folliculaire, tandis que les lutéocytes thécaux se répartissent en couronne autour de l'îlot précédent.

Ces formations glandulaires de supplémentation semblent avoir une période d'activité rela- 
tivement courte. A côté d'éléments en pleine activité, on observe en effet, de façon courante, de nombreuses formations à des stades variables de leur involution.

Bien que fréquents, remplacement et supplémentation des corps gestatifs en voie de régression ne sont pas des phénomènes obligatoires. Deux fois sur 263 observations, nous avons en effet observé sur des femelles respectivement au $6^{\mathrm{e}}$ et au $8^{\circ}$ mois de la gestation des corps gestatifs primaire et secondaire en état de dégénérescence avancée sans qu'aucun des deux phénomènes de compensation se produisent. Chez le zébu comme dans nombre d'autres espèces, les corps gestatifs ne sont pas indispensables à la poursuite normale de la dernière phase de la gestation.

\section{II.D.) Les périodes de repos sexuel ou ancestrus}

Pendant une grande partie de l'année, les cycles astraux de la femelle zébu se succèdent sans interruption. Leur durée est comprise entre 19 et 24 jours $(21,5 \pm 0,5$ jours en moyenne pour le zébu gobra).

Ce rythme régulier de l'activité sexuelle est souvent interrompu par des périodes de repos ou ANGSTRUS.

Le phénomène se traduit histologiquement par l'absence dans les deux ovaires de tout organite pouvant être rapporté à un cycle en cours: gros follicule cavitaire, follicule mûr ou déhiscent et corps jaune cyclique en phase d'activité ou en début d'involution. L'état de régression du plus récent corps progestatif permet d'évaluer le temps qui s'est écoulé entre le dernier cestrus et le jour de l'observation. Par l'examen microscopique, nous avons ainsi noté la présence d'anœstrus dont la durée varie de celle d'un cycle (corps jaune en dégénérescence ou en début d'involution) à trois mois (disparition totale des corps progestatifs).

Le fonctionnement génital de la femelle zébu dans les conditions habituelles de l'élevage en zone tropicale sèche est donc intermittent. Bien qu'influencé par les saisons, il n'est pas strictement saisonnier. Ces périodes de repos sexuel peuvent en effet se produire à n'importe quel moment de l'année. Très fréquentes pendant la saison sèche, elles ne sont cependant pas rares pendant la saison des pluies pourtant particulièrement favorable à la reproduction.

Au cours des phases d'ancestrus, les ovaires peuvent rester au repos ou au contraire manifester une importante activité.

L'ancestrus avec repos ovarien est caractérisé par la disparition de la phase de croissance folliculaire qui amène un organite du stock des petits follicules cavitaires à la maturation puis à la déhiscence. L'activité folliculaire se traduit alors par la transformation périodique de quelques follicules primaires en follicules cavitaires petits et moyens et par la transformation de ceux-ci en follicules atrétiques.

L'anestrus accompagné d'activité ovarienne est au contraire caractérisé par une importante activité évolutive des follicules cavitaires petits et moyens. Dans un ou dans les deux ovaires, un grand nombre d'entre eux débutent périodiquement leur phase de croissance. Ils grossissent et donnent à la glande un aspect polykystique mais ne terminent jamais leur évolution. Au stade grand follicule cavitaire, ils dégénèrent tous brutalement sous la forme lamelleuse et disparaissent rapidement.

La gonade de la femelle zébu en «Anœstrus avec activité ovarienne » et l'ovaire de la femme atteinte de «Maladie polykystique » ont des aspects voisins. Cette analogie structurale suggère une analogie causale. Le mécanisme pathogénique le plus souvent invoqué pour la femme est une activité hypophysaire gonadotrope F.S.H. normale associée à une insuffisance ou à un arrêt de l'activité gonadotrope L.H. Les organites évoluent mais ne peuvent atteindre les stades de la maturité et de la déhiscence. Le même mécanisme pourrait intervenir chez le zébu. Dans les anœstrus de ce type, en effet, une très nette activité des cellules F.S.H. de l'hypophyse, non accompagnée d'activité correspondante des cellules L.H., a été observée. 

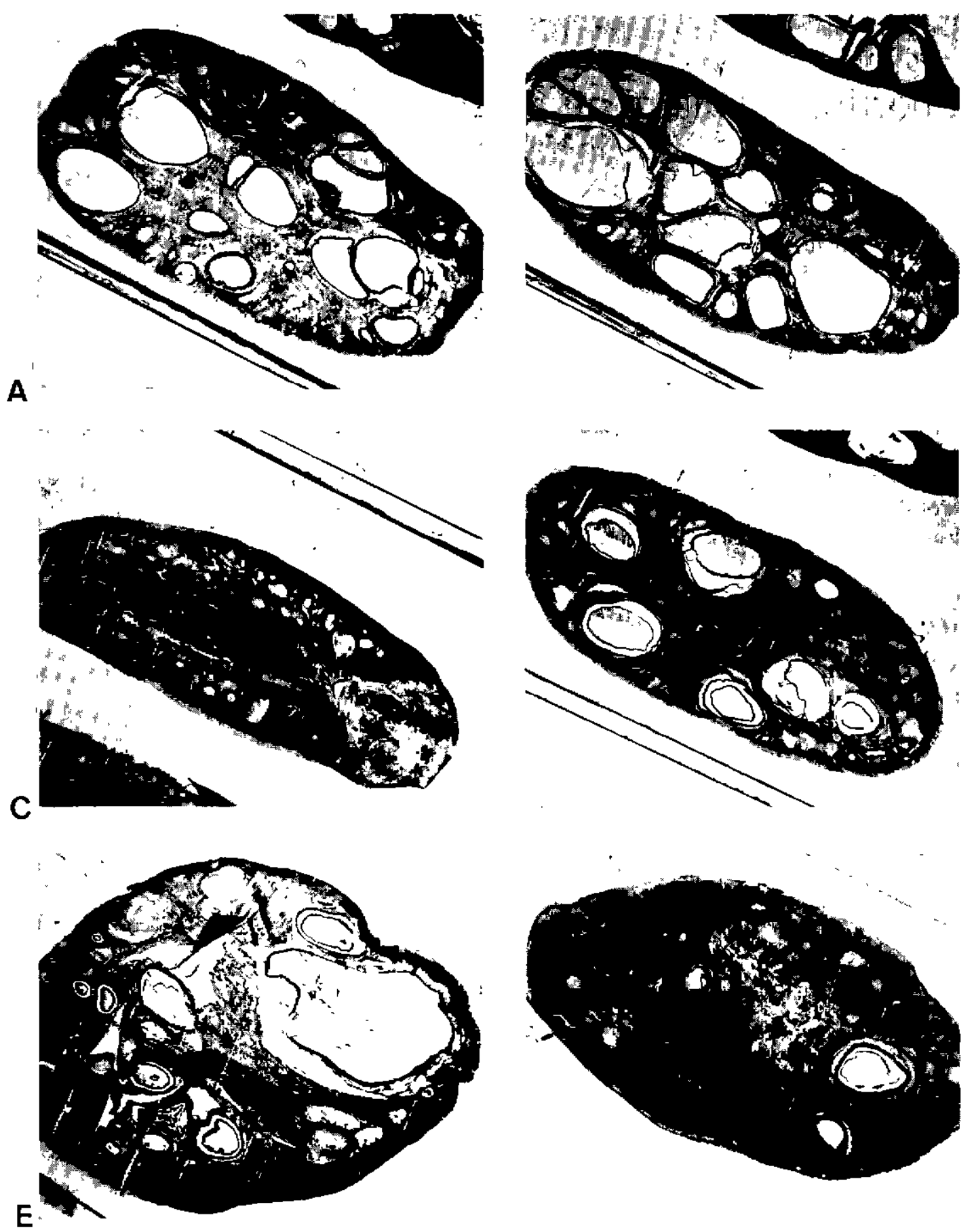

$\mathbf{F}$

Fig. 5.4. - Structure de l'ovaire de la femelle zébu.

A et B: Ancestrus long (noter sur les deux ovaires l'absence de tout organite d'origine cyclique et la forte activité folliculaire qui se traduit par la présence de nombreux follicules involutifs de type lamelleux) $(\times 12)$.

$\mathrm{C}$ et D: Ancestrus court (noter en C, sur l'ovaire gauche, le dernier corps progestatif d'origine cyclique en phase de générescence avancée et en $D$, en zone parenchymateuse de l'ovaire droit, un corps blanc cyclique) $(\times 12)$.

$\mathrm{E}$ et $\mathrm{F}$ : Anoestrus de durée moyenne (noter en $\mathrm{E}$ la présence de follicules involutifs nombreux et en $F$ celle d'un corps blanc d'origine cyclique) $(\times 12)$. 


\section{Chapitre II}

\section{La portion tubulaire ou gestative des organes génitaux de la femelle Zébu}

La portion tubulaire ou gestative des organes génitaux de la femelle assure la protection et la nutrition de l'œuf puis de l'embryon et du foetus. Lorsque ce dernier a terminé son développement, elle est l'agent actif de son élimination au moment du part.

Elle comprend : les oviductes et l'utérus.

\section{LES OVIDUCTES}

Les OVIDUCTES ou TROMPES UTERINES (Tubae uterinae) recueillent l'ovule libéré par l'ovaire au moment de l'œstrus et, après la fécondation, assurent le transit de l'œuf jusqu'à l'utérus où s'effectue la gestation. Après le coït, ils sont parcourus par les spermatozoïdes en voie de migration vers le pavillon de la trompe.

\section{MORPHOLOGIE}

Chaque oviducte est un tube flexueux dont une extrémité évasée est orientée vers l'ovaire tandis que l'autre s'unit à l'extrémité craniale de la corne utérine correspondante.

\section{I.A.) Conformation extérieure}

L'oviducte de la femelle zébu mesure 7 à $10 \mathrm{~cm}$ de longueur. Dans sa portion moyenne. son diamètre est régulier. Il varie selon les sujets de 2 à $5 \mathrm{~mm}$.

On reconnaît à l'oviducte une portion moyenne, tubulaire, et deux extrémités.

\section{I.A.1.) L'EXTREMITE OVARIENNE}

L'EXTREMITE OVARIENNE ou PAVILLON DE LA TROMPE UTERINE (Infundibulum tubae uterinae) est évasée en une sorte d'entonnoir situé dans la portion craniale de la bourse ovarique. Sa grande ouverture est orientée caudalement, vers l'ovaire. Sa portion rétrécie se prolonge par l'ampoule tubaire.

Le bord libre du pavillon est découpé en languettes inégales, les FRANGES DU PAVILLON (Fimbria tubae) qui sont toutes libres, sauf une qui se fixe sur l'ovaire et constitue le LIGAMENT TUBO-OVARIQUE ou FRANGE OVARIENNE (Fimbria ovarica).

La face externe du pavillon est libre et tapissée par la séreuse péritonéale réfléchie de la face interne du Mésosalpinx.

La face interne du pavillon est revêtue par la muqueuse tubaire, plissée radiairement. Séreuse externe et muqueuse interne se raccordent sans transition sur le bord libre des franges.

Le fond du pavillon est percé d'un orifice, l'ORIFICE ABDOMINAL DE LA TROMPE UTERINE (Ostium abdominale tubae uterinae) qui communique avec la lumière de la portion tubulaire de l'oviducte.

Il n'est pas rare d'observer chez le zébu une ou plusieurs formations vésiculaires pédiculées, fixées sur le pavillon de la trompe ou les parties voisines des parois de la bourse ovarique. Ces formations ou APPENDICES VESICULAIRES (Appendices vesiculosae) correspondent à des vestiges du mésonéphros et du canal mésonéphrotique. 


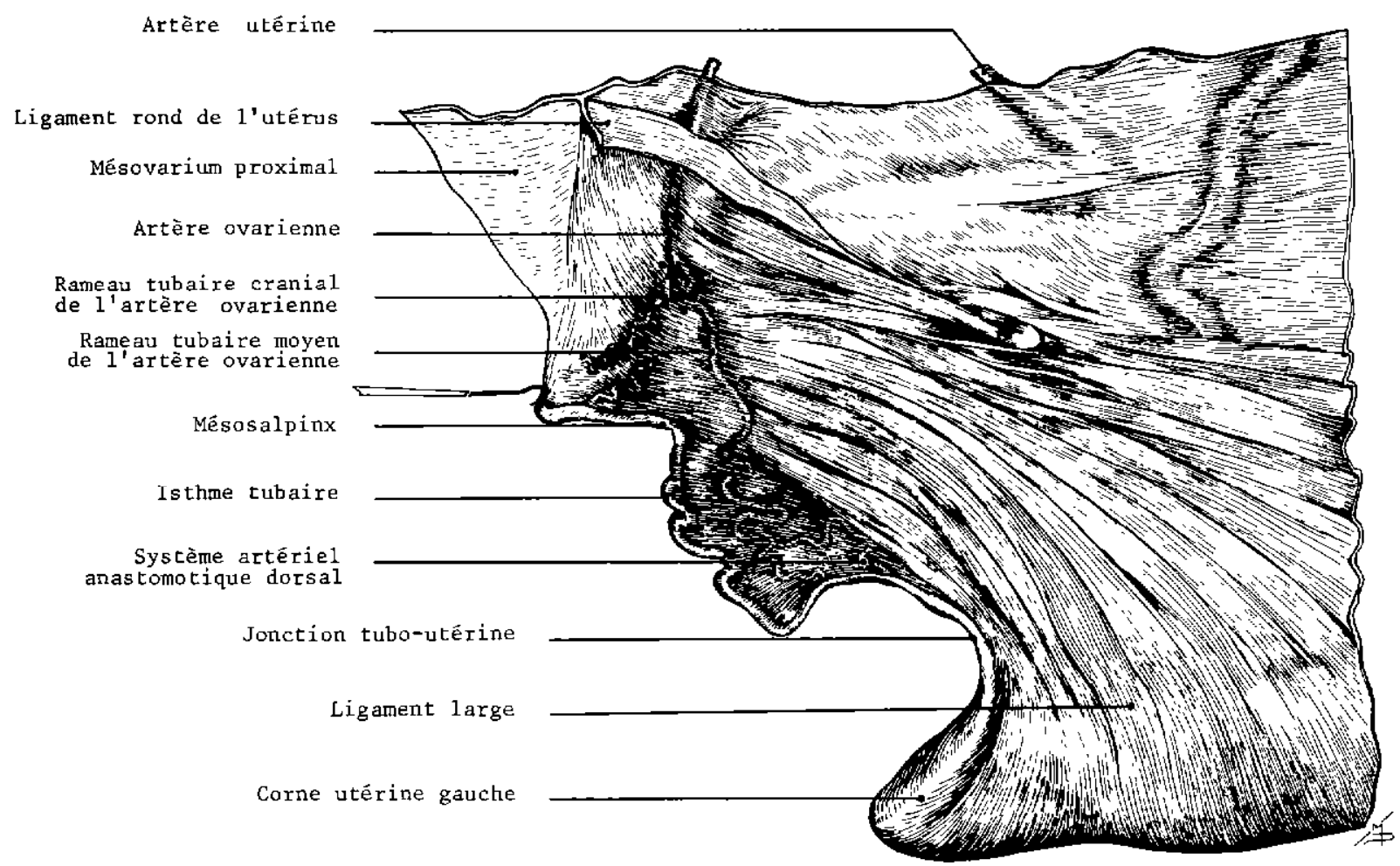

L'oviducte et la corne utêrine gauches de la femelle zêbu (vue latêrale)

\section{I.A.2.) LA PORTION MOYENNE}

LA PORTION MOYENNE ou CORPS DE L'OVIDUCTE débute par une courte portion conique, l'AMPOULE TUBAIRE (Ampulla tubae uterinae) qui fait transition entre le pavillon et l'ISTHME TUBAIRE (Isthmus tubae uterinae). Ce dernier représente la plus grande partie de l'oviducte. Il est de calibre régulier et parcourt le mésosalpinx selon un trajet sinueux.

\section{I.A.3.) LA PORTION UTERINE}

LA PORTION UTERINE (Pars uterina) ou JONCTION TUBO-UTERINE se traduit extérieurement par une augmentation progressive du diamètre de l'oviducte qui s'unit insensiblement à l'extrémité effilée de la corne utérine.

L'oviducte, selon la comparaison classique peut être assimilé à un canal excréteur en discontinuité avec sa glande. Cette particularité anatomique est évoquée par de nombreux auteurs pour expliquer les variétés abdominale et pelvienne des gestations extra-utérines et la fréquence de l'extension au péritoine des salpingites et des métrites. Il est certain que chez le zébu, au moins dans le premier cas, cette proposition doit être nuancée de réserves, en raison de l'espace relativement clos que constitue la bourse ovarique et de la présence, autour de l'orifice de cette formation, de nombreuses fibres musculaires lisses qui peuvent provoquer sa fermeture complète.

\section{I.B.) Conformation intérieure}

La lumière de l'oviducte est presque entièrement occupée par des relèvements longitudinaux de la muqueuse: les PLIS TUBAIRES (Plicae tubariae).

Ceux-ci sont particulièrement nombreux dans la région de l'ampoule où ils se subdivisent en plis secondaires et tertiaires et s'alignent dans le prolongement des plis de la face interne 
du pavillon. Dans l'isthme tubaire, le nombre et la hauteur des plis décroissent dans le sens cranio-caudal. A la jonction tubo-utérine, leur disparition marque l'emplacement de l'ORIFICE UTERIN DE L'OVIDUCTE (Ostium uterinum tubae).

\section{MOYENS DE FIXITE, TOPOGRAPHIE, RAPPORTS}

L'oviducte est uni à l'ovaire par l'une des franges de son pavillon : LE LIGAMENT TUBO-OVARIQUE ou FRANGE OVARIENNE (Fimbria ovarica); il est en continuité avec l'utérus au niveau de la JONCTION TUBO-UTERINE. Sur toute sa longueur, il est en outre fixé par son méso propre : le MESOSALPINX.

Le pavillon est situé dans la bourse ovarique, cranio-latéralement par rapport à l'ovaire. Il est en relief sur la face profonde du mésosalpinx.

Le reste de l'oviducte est compris entre les deux lames séreuses de son méso de suspension. Après un très court trajet cranial, l'organe se replie caudo-ventralement et, à distance du bord libre du méso, décrit une courbe à convexité ventrale avant de s'unir à la corne utérine. Tout le long de son parcours, l'oviducte décrit des sinuosités assez lâches.

Solidaire de la bourse ovarique et de l'ovaire, l'oviducte prend avec les organes abdominopelviens les mêmes rapports que la glande.

\section{STRUCTURE}

Structuralement, l'oviducte est composé de trois tuniques : une séreuse, une musculeuse et une muqueuse.

\section{III.A.) La tunique séreuse}

La TUNIQUE SEREUSE (Tunica serosa) comprend la lame séreuse épithéliale et la lame sous-séreuse.

\section{III.A.1.) LA LAME SEREUSE EPITHELIALE}

La LAME SEREUSE EPITHELIALE (Lamina epithelialis serosae) est formée par l'épithélium endothéliforme du péritoine. Elle recouvre la face excentrique du pavillon et les faces de la portion moyenne et de la jonction tubo-utérine dont les bords sont en rapport avec la lame conjonctive interséreuse du mésosalpinx.

\section{III.A.2.) LA LAME SOUS-SEREUSE}

La LAME SOUS-SEREUSE (Lamina subserosa), conjonctivo-élastique, est caractérisée par la présence de fibres musculaires lisses, plexiformes, qui s'irradient des bords de la trompe vers le mésosalpinx et le mésovarium proximal. Elles constituent une véritable « MUSCULATURE SOUS-PERITONEALE » capable de mobiliser l'organe par rapport à l'ovaire et de permettre au pavillon de coiffer la glande au moment de l'ovulation.

\section{III.B.) La tunique musculeuse}

La TUNIQUE MUSCULEUSE (Tunica muscularis), formée de fibres lisses, est constituée, comme tous les dérivés du canal de Muller, d'une couche longitudinale superficielle (Stratum longitudinale) et d'une couche circulaire profonde (Stratum circulare) séparées par une couche intermédiaire conjonctivo-vasculaire peu épaisse.

Réduite à quelques fibres diversement orientées dans le pavillon, cette tunique musculeuse est d'autant plus développée qu'on se rapproche de la corne utérine. La couche longitudinale externe en particulier, discontinue au niveau de l'ampoule et de l'isthme, est particulièrement épaisse à la jonction tubo-utérine. 
Cette « MUSCULATURE PROPRE DE L'OVIDUCTE » dont l'action complète celle de la « MUSCULATURE SOUS-PERITONEALE » est capable d'animer l'organe de mouvements péristaltiques et contribue au transport de l'œuf vers l'utérus.

\section{III.C.) La tunique muqueuse}

La TUNIQUE MUQUEUSE (Tunica mucosa) adhère à la face interne de la musculeuse. Elle se relève vers la lumière du conduit pour constituer les PLIS TUBAIRES. Elle est composée d'un chorion conjonctif et d'un épithélium unistratifié.

\section{III.C.1.) LE CHORION OU PROPRIA}

Le CHORION ou PROPRIA (Lamina propria mucosae) est formé de fibres conjonctives lâches, entrelacées et de cellules conjonctives fixes ou mobiles. Il est dépourvu de glande, mais renferme un riche réseau capillaire sanguin et un important plexus lymphatique.

Le réseau sanguin artériel et veineux est particulièrement important au niveau du pavillon dont il transforme le chorion en une sorte de tissu érectile qui favorise l'action de la musculature sous-péritonéale au moment de l'ovulation.

Le plexus lymphatique chorial est constitué par des fentes lymphatiques intercommunicantes très développées qui envahissent, outre la couronne marginale, les lames principales et les lamelles de premier et second ordre.

\section{II.C.2.) L'EPITHELIUM}

L'EPITHELIUM (Lamina epithelialis mucosae) est simple. Il est composé de deux types principaux de cellules, des cellules ciliées et des cellules non ciliées dont la répartition se modifie avec l'âge, le cycle œstral et les divers segments de l'oviducte.

\section{III.C.2.a) Les cellules ciliées}

LES CELLULES CILIEES (Epitheliocytus ciliatus), prismatiques ou cubiques, sont pourvues de cils longs et animés de mouvements qui participent au transit de l'œuf en direction de l'utérus.

\section{III.C.2.b) Les cellules non ciliées}

LES CELLULES NON CILIEES (Epitheliocytus nonciliatus) sont principalement représentées par des CELLULES SECRETOIRES. Ces dernières sont ovalaires. Leur apex est souvent garni d'un «plateau strié ». Certaines renferment dans la portion supra-nucléaire de leur cytoplasme des produits de sécrétion colorables par l'A.P.S. (Acide periodique de Schiff) qui jouent un rôle nutritif à l'égard de l'œuf et agissent sur les spermatozoïdes en parachevant leur maturation fertilisatrice.

Certaines cellules non ciliées sont dépourvues de produits de sécrétion et sont généralement considérées comme des CELLULES GLANDULAIRES AU REPOS. Un certain nombre d'entre elles correspondrait cependant à des CELLULES ABSORBANTES qui, réparties sur les franges du pavillon et les plis de l'ampoule, dirigeraient au moment de l'ovulation l'abondante sérosité péritonéale de la bourse ovarique et la liqueur folliculaire vers les fentes lymphatiques du chorion et assureraient ainsi la captation de l'ovule.

A certains moments du cycle, on peut observer dans l'épithélium tubaire des cellules étroites dites "en bâtonnets ", certaines ont un apex saillant, au-dessus des pôles apicaux des cellules voisines. Elles sont considérées comme des cellules sécrétrices entièrement vides et des formes d'expulsion de cellules sénescentes. 

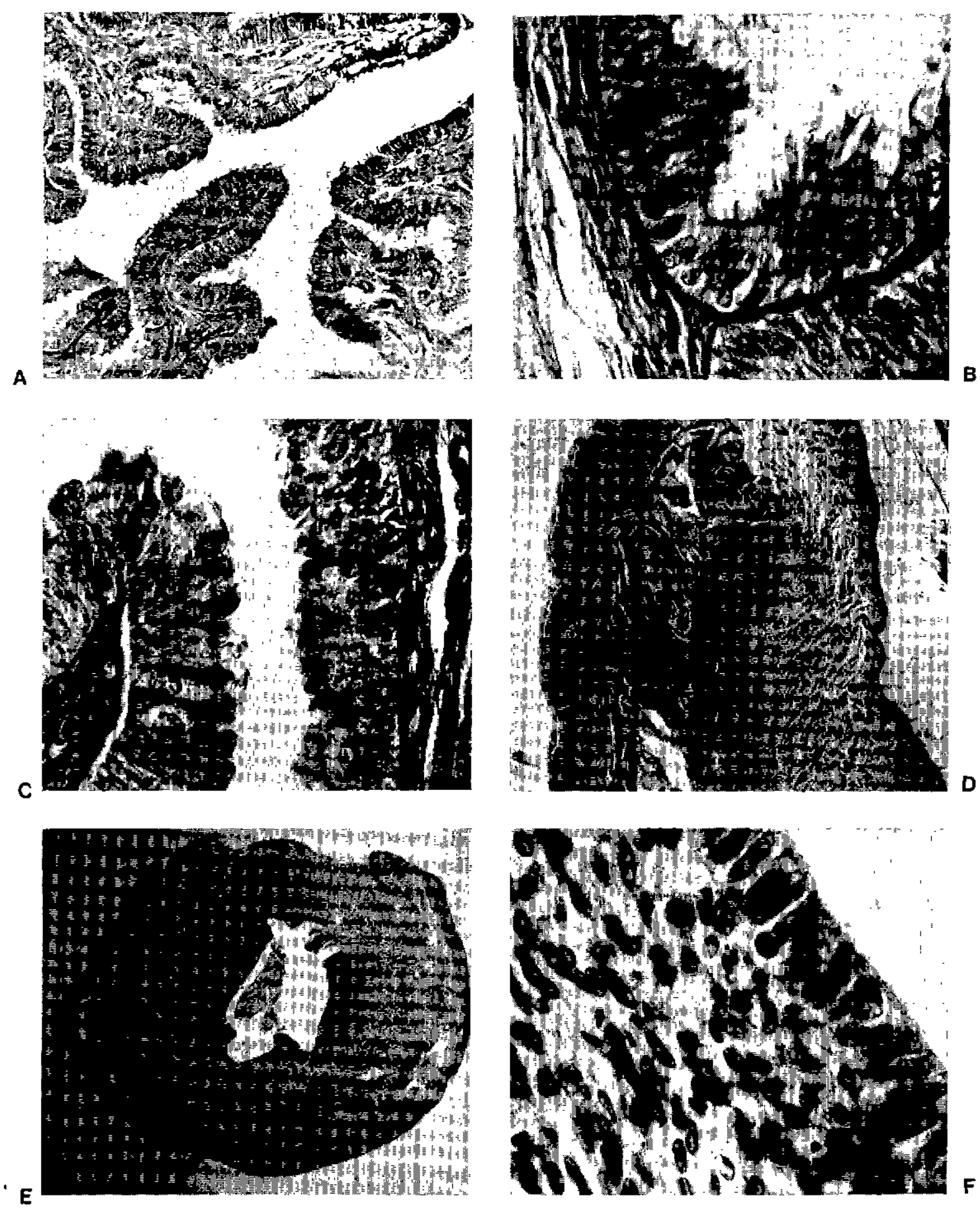

Fig. 7. - Structure de l'oviducte et de l'utérus de la femelle zébu.

A) Plis tubaires de l'isthme tubaire (P.A.S. - orange G.; $\times 250$; noter la présence de cellules non ciliées sécrétoires à apex foncé).

B) Cellules ciliées de l'épithélium tubaire pendant la phase cestrogénique du cycle cestral (X 1.000).

C) Cellules non ciliées sécrétoires de l'épithélium tubaire pendant la première moitié de la phase progestative du cycle cestral $(\times 1.000)$.

D) La paroi utérine au début du post ostrus $(\times 8)$.

E) Coupe transversale de l'extrémité tubaire de la corne utérine (à droite et à gauche caroncules à stroma dépourvu de glandes) ( $\times 12$ ).

F) Epithélium et chorion utérin pendant le di-œestrus $(\times 1.000)$. 


\section{III.C.2.c) Variations}

Le rapport quantitatif entre cellules ciliées et cellules non ciliées de l'épithélium tubaire varie avec la région, le stade du cycle cestral et l'âge de l'animal.

Les cellules ciliées sont plus nombreuses dans le pavillon et l'ampoule que dans l'isthme et la portion utérine.

En phase cestrogénique du cycle, les cellules ciliées sont plus nombreuses et plus hautes tandis que les cellules sécrétrices présentent le même phénomène dans la première moitié de la phase progestative.

Avec l'âge, le nombre des cellules ciliées diminue, la tunique musculaire s'amincit, les plis muqueux régressent et la lumière de l'oviducte se rétrécit.

\section{VAISSEAUX ET NERFS}

\section{IV.A.) Les artères de l'oviducte}

La jonction tubo-utérine est irriguée par le rameau utérin de l'artère ovarienne. Le pavillon, l'ampoule et l'isthme tubaire reçoivent le sang des rameaux tubaires qui s'anastomosent entre eux pour constituer deux systèmes anastomotiques, l'un dorsal et l'autre ventral.

\section{IV.A.1.) LE RAMEAU UTERIN DE L'ARTERE OVARIENNE}

Le rameau utérin de l'artère ovarienne (Ramus uterinus) encore appelé artère utérine crâniale, artère tubo-utérine ou artère salpingo-utérine est une branche collatérale de l'artère ovarienne. Il naît du bord caudal de cette dernière et se dirige ventro-caudalement vers la jonction tubo-utérine dans laquelle il se termine par deux branches qui échangent entre elles une anastomose avant d'atteindre l'organe.

La branche terminale caudale émet un rameau anastomotique vers le rameau crânial de l'artère utérine.

La branche terminale crâniale délègue par son bord rostral le rameau tubaire caudal.

\section{IV.A.2.) LES RAMEAUX TUBAIRES}

Les RAMEAUX TUBAIRES (Rami tubarii) ou ARTERES TUBAIRES, au nombre de trois, sont distingués en crânial, moyen et caudal.

\section{IV.A.2.a) Le rameau tubaire cranial}

Le RAMEAU TUBAIRE CRANIAL est une branche collatérale de l'artère ovarienne. Il naît de son bord rostral, en amont du point d'émission du rameau utérin. Entre les lames séreuses du mésovarium puis du mésosalpinx, il se dirige ventralement vers le pavillon qu'il irrigue.

Près de sa terminaison, il émet deux branches collatérales: une branche anastomotique caudale qui rejoint le rameau tubaire moyen et une grêle branche crâniale qui longe le bord ventral, convexe, de l'oviducte et s'anastomose avec la première collatérale ventrale du rameau tubaire caudal pour former le SYSTEME ANASTOMOTIQUE VENTRAL.

\section{IV.A.2.b) Le rameau tubaire moyen}

Le RAMEAU TUBAIRE MOYEN naît du bord rostral de l'artère ovarienne, un peu en aval du rameau utérin. Il s'engage dans le mésosalpinx, reçoit au niveau du pavillon une branche anastomotique du rameau tubaire crânial puis longe le bord dorsal, concave, de l'isthme tubaire pour s'anastomoser avec le rameau tubaire caudal en formant le SYSTEME ANASTOMOTIQUE DORSAL. 


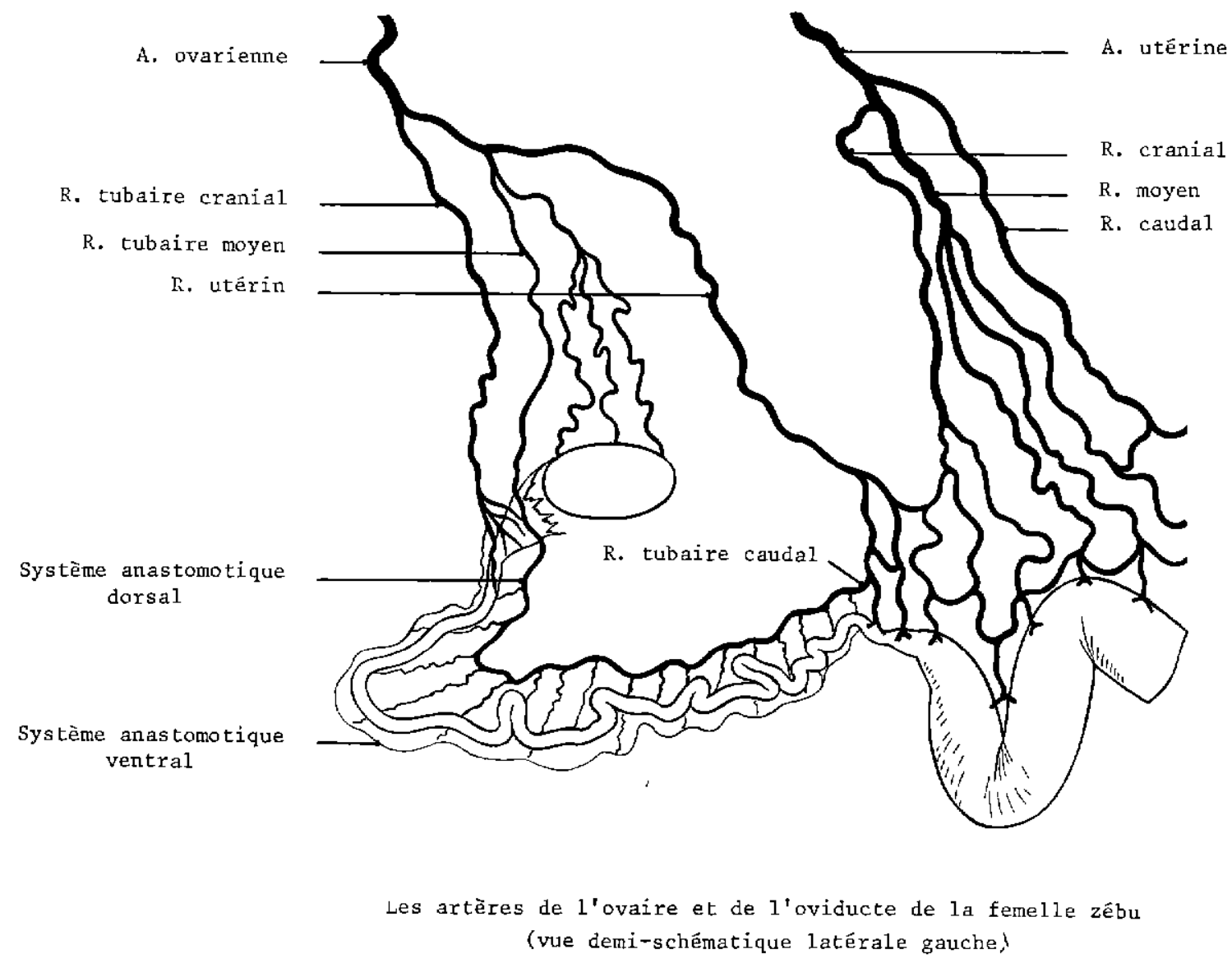

\section{IV.A.2.c) Le rameau tubaire caudal}

Le RAMEAU TUBAIRE CAUDAL naît de la branche terminale crâniale du rameau utérin. Il longe le bord dorsal de l'oviducte et s'anastomose avec le rameau tubaire moyen pour former le SYSTEME ANASTOMOTIQUE DORSAL.

Sa première collatérale ventrale longe le bord ventral de l'oviducte et s'anastomose avec une branche de l'artère tubaire crâniale pour former le SYSTEME ANASTOMOTIQUE VENTRAL.

\section{IV.A.3.) LE SYSTEME ANASTOMOTIQUE DORSAL}

Le système anastomotique dorsal est bien développé. Il se constitue à partir des rameaux tubaires moyen et caudal. Il longe la concavité de l'oviducte et s'y distribue par 15 à 20 branches de dimension moyenne.

\section{IV.A.4.) LE SYSTEME ANASTOMOTIQUE VENTRAL}

Le système anastomotique ventral grêle, souvent absent, résulte de l'anastomose des rameaux tubaires crânial et caudal. Il se distribue à l'oviducte par 8 à 10 petites branches.

\section{IV.B.) Les veines tubaires}

Le sang de l'oviducte est drainé par une arcade veineuse homologue du système artériel anastomotique dorsal. Cette arcade résulte de l'anastomose des veines tubaire crâniale et tubaire caudale. 


\section{IV.B.1.) L'ARCADE VEINEUSE DORSALE}

L'ARCADE VEINEUSE DORSALE longe le bord dorsal de l'oviducte. Elle est satellite du système artériel anastomotique dorsal et reçoit de l'oviducte 6 à 8 affluents.

Cranialement, cette arcade se prolonge par la VEINE TUBAIRE CRANIALE. Caudalement, elle s'unit à la VEINE TUBAIRE CAUDALE.

\section{IV.B.2.) LA VEINE TUBAIRE CRANIALE}

La VEINE TUBAIRE CRANIALE prolonge rostralement l'arcade veineuse dorsale. Elle est satellite du rameau artériel tubaire cranial et se jette dans la veine ovarienne dont elle est le dernier affluent.

Elle reçoit par son bord rostral une VEINE TUBAIRE VENTRALE grêle et courte qui peut être considérée comme l'ébauche d'une arcade veineuse ventrale.

Elle reçoit par son bord caudal une VEINE INFUNDIBULAIRE qui draine le pavillon.

\section{IV.B.3.) LA VEINE TUBAIRE CAUDALE}

La VEINE TUBAIRE CAUDALE prolonge aboralement l'arcade veineuse dorsale. Elle s'unit à la VEINE UTERINE CRANIALE pour former la VEINE TUBO-UTERINE, très courte, qui rejoint la VEINE MARGINALE DE L'UTERUS.

\section{IV.C.) Les lymphatiques de l'oviducte}

Les VAISSEAUX LYMPHATIQUES de l'oviducte naissent du système des fentes lymphatiques choriales. Ils forment des réseaux périvasculaires qui rejoignent les GANGLIONS ILIAQUES MEDIAUX (Lymphodoni iliaci mediales).

\section{IV.D.) Les nerfs de l'oviducte}

Les NERFS DE L'OVIDUCTE appartiennent au système nerveux autonome. Ils sont d'abord mêlés aux nerfs du plexus ovarien, et Iongent les gros vaisseaux. Ils se distribuent en filets moteurs pour les musculatures et les vaisseaux et en filets sensitifs.

\section{L'UTERUS}

L'utérus est un sac musculo-membraneux, situé à la limite des cavités pelvienne et abdominale. Uni rostralement à l'oviducte et caudalement au vagin, il est, après le coït, le lieu de passage des spermatozoïdes en migration vers le pavillon de l'oviducte. Plus tard, après la fécondation, il reçoit l'œuf et assure la croissance de l'embryon puis celle du fœtus qu'il expluse au moment du part.

Par sa morphologie, sa topographie, sa structure et son innervation, l'utérus de Bos indicus présente d'importantes particularités qui le distinguent de celui de Bos taurus.

\section{MORPHOLOGIE}

L'utérus de la femelle zébu est caractérisé par l'extrême réduction de son corps utérin. Il appartient de ce fait à la catégorie dite Uterus bipartitus.

\section{I.A.) Conformation extérieure}

L'examen de l'organe entier, après isolement, montre qu'en raison de l'interférence du vagin sur le col utérin et de la réduction du corps de l'utérus, les cornes seules sont visibles dans la grande majorité des cas. 


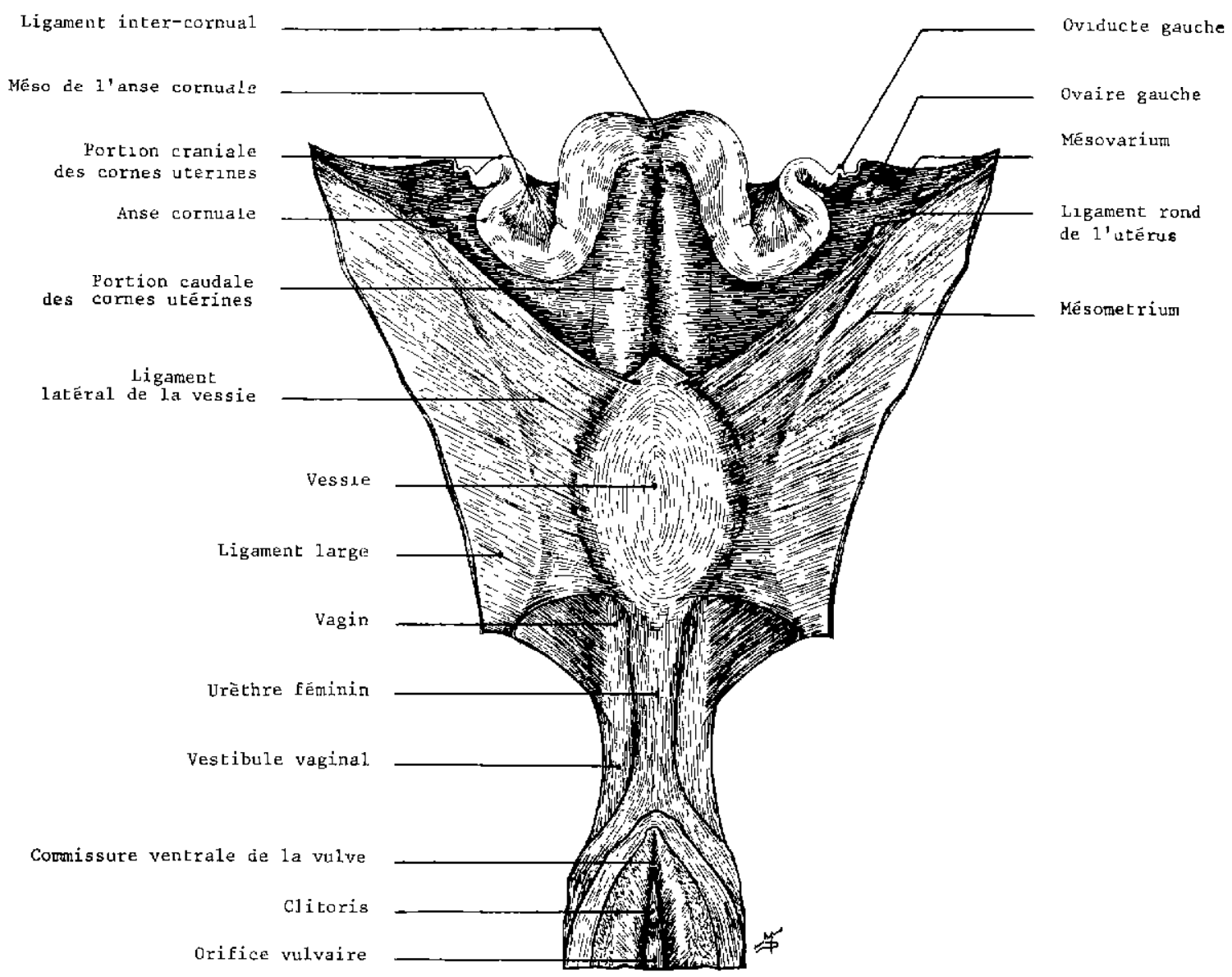

Conformatıon extérieure des organes génitaux de la femelle zébu (vue ventrale)

LES CORNES UTERINES (Cornu uteri dextrum et sinistrum) placées côte à côte émergent cranialement du bourrelet ciculaire que forme l'interférence vaginale sur le col utérin. Elles se disposent ensuite selon un trajet complexe et sinueux qui les porte successivement ventralement, caudalement, dorsalement puis rostro-médialement.

Isolées et débarrassées de leur méso de suspension, les cornes utérines forment chacune un conduit membraneux de consistance moyenne dont le diamètre diminue progressivement dans le sens caudo-cranial. Chez la femelle pubère mais nullipare qui sert de base à notre étude, elles mesurent 10 à 12 centimètres de longueur. Leur diamètre passe de 1 à $2 \mathrm{~cm}$ à la sortie du bourrelet vaginal circulaire à celui des portions utérines de l'oviducte avec lesquelles elles s'unissent de façon insensible.

En place, suspendues par leur méso, les cornes utérines ont une disposition spaciale particulière qui permet de les diviser en trois portions.

\section{I.A.1.) LA PORTION CAUDALE OU RECTILIGNE DES CORNES UTERINES}

La PORTION CAUDALE ou RECTILIGNE DES CORNES semble se dégager directement du bourrelet vaginal. Etroitement accolées l'une à l'autre par un feuillet séreux qui les enveloppe en commun, les portions correspondantes des deux cornes se disposent caudo-rostra- 
lement selon un trajet horizontal ou légèrement oblique ventralement qui mesure 4 à $5 \mathrm{~cm}$ de longueur.

Les faces médiales de la portion rectiligne des cornes sont unies par du tissu conjonctif. Leurs faces latérales sont libres et recouvertes par la séreuse péritonéale qui, des bords dorsaux et ventraux, se réfléchit directement d'une corne à l'autre.

Le BORD VENTRAL reçoit en outre l'insertion du ligament large et mérite pour cette raison le nom de BORD FIXE ou MESOMETRIQUE (Margo mesometrium) tandis que le BORD DORSAL, dépourvu d'insertion, peut être appelé BORD LIBRE (Margo liber).

\section{I.A.2.) LA PORTION MOYENNE DES CORNES UTERINES OU ANSE CORNUALE}

LA PORTION MOYENNE DES CORNES UTERINES ou ANSE CORNUALE prolonge caudo-latéralement et ventralement la portion rectiligne. Elle mesure 3 à $4 \mathrm{~cm}$.

Dans cette portion, chaque corne, repliée en boucle sur elle-même, forme une anse dont la concavité est occupée par une dépendance du ligament large qui en solidarise étroitement les différentes portions.

La moitié craniale de l'anse est oblique ventralement, latéralement et caudalement. Elle est séparée de la portion rectiligne par une courbure à concavité aborale. Dans l'angle de séparation des deux cornes, la séreuse forme un petit méso triangulaire simple appelé LIGAMENT INTERCORNUAL (Ligamentum intercornuale).

La moitié caudale de l'anse cornuale est oblique dorsalement, médialement et souvent légèrement cranialement. Son extrémité dorsale est en rapport avec la face latérale de la portion rectiligne de la corne correspondante tandis que son extrémité ventrale, en continuité avec la moitié craniale de l'anse, forme avec elle une courbure à concavité plus ou moins large, orientée dorsalement.

\section{I.A.3.) LA PORTION CRANIALE OU TUBAIRE DES CORNES UTERINES}

La PORTION CRANIALE OU TUBAIRE des cornes utérines prolonge la moitié caudale de l'anse cornuale. Disposée à peu près horizontalement, elle mesure 3 à $4 \mathrm{~cm}$ de longueur, se dirige rostro-médialement puis caudalement selon un trajet plus ou moins sinueux. Elle prend souvent la forme d'une épingle à cheveux à convexité craniale dont la branche externe prolonge rostralement l'anse cornuale tandis que la branche interne par son extrémité caudale effilée s'unit insensiblement à la portion utérine de l'oviducte.

Cette partie des cornes utérines est suspendue à la portion correspondante du ligament large par son bord dorsal qui devient donc le bord fixe ou Mésométrique tandis que les faces et le bord ventral (qui devient le bord libre) sont dépourvus d'insertion ligamenteuse et tapissés par la séreuse péritonéale. En raison de sa disposition particulière, la portion tubaire de la corne tend à orienter médialement et caudalement la partie craniale du ligament large.

\section{I.A.4.) VARIATIONS}

La morphologie des cornes utérines varie avec les conditions physiologiques dans lesquelles se trouve l'organe.

A CHAQUE CYCLE, au moment de l'oestrus, les comes utérines deviennent turgescentes. Leur diamètre s'accroît légèrement. A la palpation, leur paroi paraît plus épaisse et plus ferme.

PENDANT LA GESTATION, la corne utérine gravide subit une augmentation de volume considérable. Elle atteint et peut dépasser, dans la période qui précède le part, un mètre de longueur. 
M. retracteur du clitoris

M. constricteur de la vulve

Cul-de-sac recto-gēnital

M. constricteur du vestibule vaginal Vagin

Cul-de-sac vesico-gênital

Commissure ventrale de la vulve

Ligament latéral de la vessie

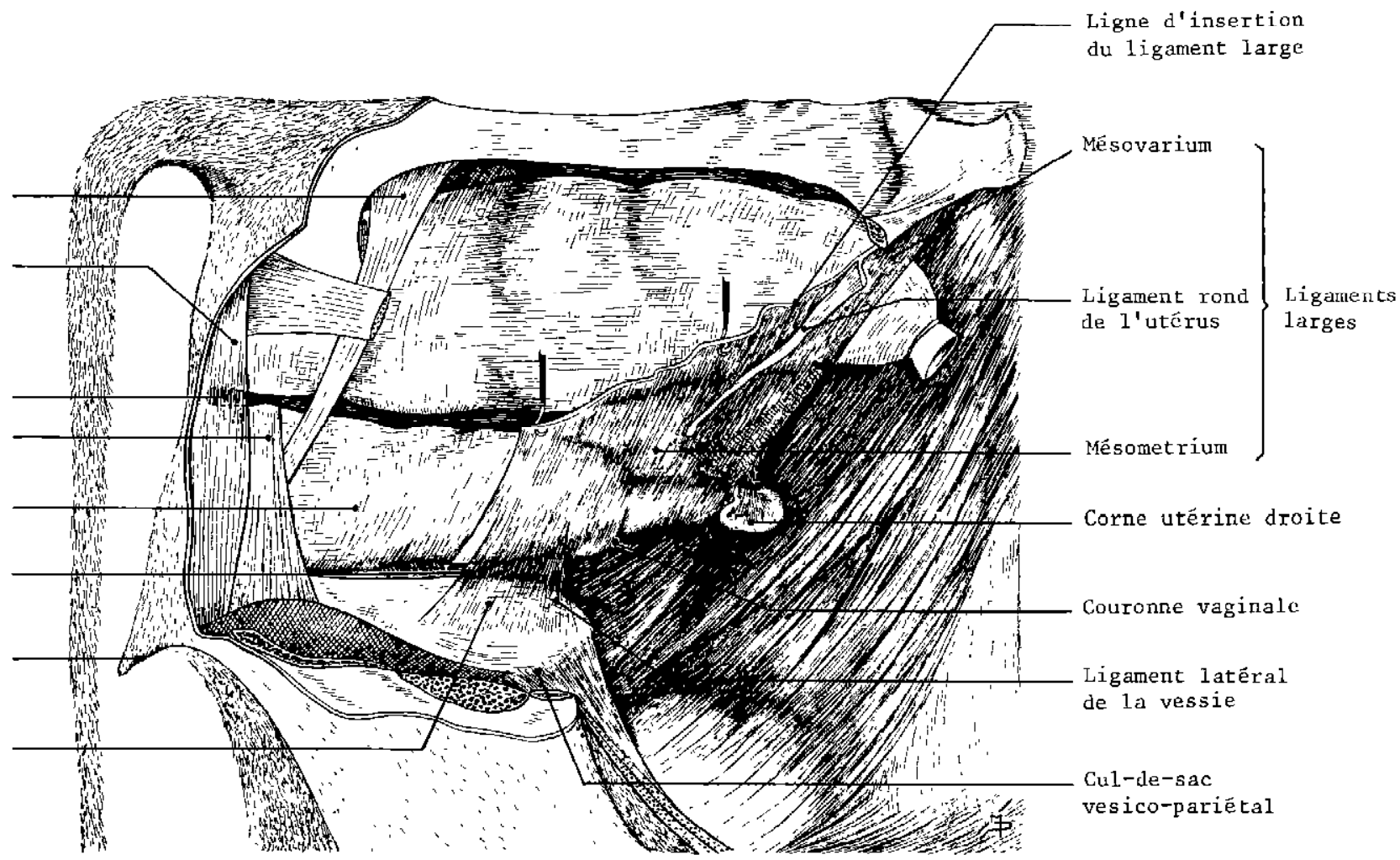

Le tractus gënital de la femelle zébu

(vue latêrale droite, organes en $\mathrm{pl}$ ace après ouverture du bùssin) 
Cette hypertrophie porte surtout sur la portion moyenne de la corne qui, fixée caudalement par sa continuité avec le vagin et cranialement par le dispositif séro-ligamenteux suspenseur de l'ovaire, s'engage de plus en plus dans la cavité abdominale et forme un volumineux croissant dont les pointes dorso-caudales moins mobiles subissent une migration et un développement beaucoup plus limités que ceux de la partie moyenne.

Après le part, la corne utérine subit une involution qui tend mais ne parvient jamais à lui redonner sa forme et ses dimensions primitives. A la suite de chaque gestation, elle présente donc une taille supérieure à celle qu'elle avait dans la période d'inter-vêlage précédent. Chez la vache multipare non gravide, les cornes utérines peuvent ainsi atteindre une longueur de 35 à $40 \mathrm{~cm}$ et un diamètre de 5 à $6 \mathrm{~cm}$. Morphologiquement, cet accroissement intéresse surtout l'anse cornuale qui de ce fait change de morphologie selon deux modalités possibles. Dans la première, le $\mathrm{V}$ formé par cette portion des cornes s'allonge caudo-ventralement et peut à la limite former une anse de 9 à $10 \mathrm{~cm}$ de long dont les branches inégales sont au contact l'une de l'autre. Dans la seconde modalité la plus fréquente, l'anse cornuale se transforme en U par allongement prépondérant de sa partie moyenne.

CHEZ LES TRES VIEILLES FEMELLES, l'utérus subit une involution progressive lorsqu'a cessé l'activité sexuelle. Il devient fibreux et diminue de volume mais garde toujours la disposition morphologique acquise lors de la période active de la vie génitale.

\section{I.B.) Conformation intérieure}

L'ouverture du bord dorsal du tractus génital permet de mettre en évidence la cavité utérine et le canal cervical dont la morphologie et les fonctions sont si particulières qu'il mérite une description à part.

LA CAVITE UTERINE (Cavum uteri) est virtuelle en dehors de la gestation. Elle est tapissée par une muqueuse jaune rosâtre qui présente des reliefs arrondis, les CARONCULES (Carunculae). Celles-ci, au nombre d'une cinquantaine, sont tassées les unes contre les autres au niveau de leur apex, et séparées par des vallées étroites à leur base. Leur volume diminue caudo-cranialement au fur et à mesure qu'on se rapproche de l'extrémité tubaire des cornes où elles ne se traduisent plus morphologiquement que par un très faible relief de la muqueuse.

Dans la plupart des espèces, la cavité utérine se divise en CAVITE DES CORNES et CAVITE DU CORPS. Chez le zébu, la cavité du corps utérin est virtuelle. Les parois médiales de la portion horizontale des cornes s'adossent en effet l'une à l'autre et forment une cloison ou VELUM UTERIN (Velum uteri) dont le bord libre ou EPERON UTERIN est au contact de l'orifice rostral du col et réduit à néant les dimensions du corps.

AU COURS DU CYCLE OESTRAL, la cavité utérine subit des modifications. La muqueuse qui la limite est rose-jaunâtre, pâle et sèche, pendant le di-oestrus. La période oestrogénique du cycle se traduit par une congestion progressive de la muqueuse qui se couvre en même temps d'un mucus de plus en plus abondant. A l'oestrus, elle est turgescente et sa coloration devient franchement rouge, parfois même violacée. Le mucus est alors clair et fluide. Pendant la période progestative du cycle, la muqueuse perd peu à peu son aspect congestif. Après une période d'exacerbation, la sécrétion utérine diminue, le mucus devient épais, consistant et jaunâtre.

PENDANT LA GESTATION, les principales modifications sont d'ordre structural mais il convient de noter au chapitre de la morphologie une nette augmentation des dimensions, du nombre et de la structure des caroncules.

Grossièrement ovoïdes, à côtés plus ou moins aplatis du fait qu'elles sont tassées les unes contre les autres, elles mesurent en moyenne $1,5 \times 1 \mathrm{~cm}$ chez la vache vide multipare. Vers le troisième mois de la gestation, leurs dimensions sont à peu près multipliées par trois. Elles atteignent 10-11 $\times 6 \mathrm{~cm}$, au sixième mois, et conservent cette taille jusqu'au part. Cet accroissement 
dimentionnel intéresse surtout l'apex caronculaire. Il est presque nul au niveau du pied, si bien qu'au fur et à mesure qu'elle grossit, cette formation se pédiculise. Il convient de noter en outre, qu'après chaque gestation les caroncules ne reprennent jamais leurs dimensions primitives, de sorte que, comme les cornes utérines, elles sont plus volumineuses chez la vache multipare que chez la génisse.

Le nombre des caroncules augmente aussi progressivement au cours de la gestation. Vers le sixième mois, il est à peu près triplé et reste à ce niveau jusqu'à la mise bas.

Structuralement enfin, les caroncules se vascularisent abondamment, leur surface se creuse de cryptes plus ou moins ramifiés dans lesquels s'engrènent les villosités des cotylédons foetaux.

CHEz LA VACHE AGEE la muqueuse est sèche, mince et pâle, de coloration grisjaunâtre. Les caroncules diminuent en nombre et en volume. Elles sont presque imperceptibles ohez les très vieux sujets.

\section{I.C.) Le col utérin}

Sur le tractus génital isolé, le COL UTERIN (Cervix uteri) est plus ou moins caché par le bourrelet vaginal. Il comprend par conséquent une PORTION VAGINALE (Partio vaginalis) toujours très importante et une portion pré-vaginale (Partio prevaginalis) réduite et parfois même inexistante.

Bien que peu visible, il peut être identifié par palpation à travers la paroi vaginale, grâce à sa consistance nettement plus ferme que celle des portions voisines du tractus génital. Sur l'animal vivant, il peut donc être reconnu par exploration rectale et maintenu en place en vue d'un cathétérisme cervical.

Après application d'un spéculum sur l'organe en place ou après ouverture du plafond du vagin sur l'organe isolé, le revers vaginal du col peut être observé. Il se présente sous l'aspect d'une masse cylindroïde de 6 à $9 \mathrm{~cm}$ de longueur et de 10 à $13 \mathrm{~cm}$ de circonférence, disposée longitudinalement dans la portion craniale du vagin. Il est tapissé d'une muqueuse rougeâtre garnie de 3 ou 4 bourrelets circulaires.

Son sommet caudal est obtu et percé d'un orifice infundibulaire : l'ORIFICE EXTERNE OU VAGINAL DU COL UTERIN (Ostium uteri externum) qui assure sa communication avec la cavité vaginale.

La cavité du col utérin est tubulaire et forme le CANAL DU COL UTERIN (Canalis cervicis uteri) ou CANAL CERVICAL. Elle est rectiligne ou faiblement sinueuse et spacieuse, mais sa lumière est presque entièrement occupée par trois ou quatre BOURRELETS CIRCULAIRES (Plicae circulares) subdivisés par des sillons longitudinaux en papilles quadrangulaires à sommet aplati.

L'ORIFICE INTERNE DU COL (Ostium uteri internum), plus étroit, est au contact de l'éperon utérin. Il assure la communication avec la cavité utérine.

Par sa disposition rectiligne, son diamètre et la forme aplatie des papilles qui garnissent sa paroi, le canal cervical du zébu peut être facilement franchi par un cathéter.

Au cours des différentes étapes de la vie génitale de la femelle, le col utérin subit des modifications :

- PENDANT LE DI-cestruS ou LES PERIOdES D'ANGSTRUS, le col utérin est ferme et son canal est étroit bien que toujours capable d'être franchi par une sonde. Sa muqueuse est pâle et sèche.

- AU PRO-(ESTRUS la muqueuse devient rougeâtre. Elle est turgescente et franchement rouge à l'ESTRUS au cours duquel le canal cervical dilaté peut laisser passer en direction du vagin une quantité plus ou moins abondante de mucus utérin. 
- AU COURS DU POST-ESTRUS, ces phénomènes rétrocèdent. Le col a l'aspect de repos lorsque commence le di-œstrus.

- APRES LA FECONDATION, le col se rétracte et se ferme par contraction de ses fibres musculaires lisses. Le canal cervical se remplit d'un mucus dense décrit sous le nom de "gélatine de Warton». A partir du $3^{\mathrm{e}}$ mois de la gestation, la longueur et la circonférence du col s'accroissent. Elles atteignent respectivement 12,5 et $28,5 \mathrm{~cm}$ vers le $6^{\mathrm{e}}$ mois et conservent ensuite cette taille. Comme la corne utérine et les caroncules, le col utérin ne reprend pas complètement ses dimensions primitives après chaque gestation. Il est par conséquent d'autant plus volumineux que la vache a donné naissance à un plus grand nombre de veaux.

\section{MOYENS DE FIXITE, RAPPORTS ET TOPOGRAPHIE DE L'UTERUS}

Outre sa continuité avec le reste du tractus génital, l'utérus est appendu à la région souslombaire et aux faces latérales du bassin par les ligaments larges, qui lui confèrent sa topographie et ses rapports.

\section{II.A.) Ligament large}

Chaque LIGAMENT LARGE (Lig. latum uteri) est une vaste lame conjonctive triangulaire dont les faces sont tapissées par la séreuse péritonéale. Morphologiquement, on reconnaît à chacun d'eux trois bords, trois angles et deux faces.

\section{II.A.1.) BORD DORSAL}

Le BORD DORSAL ou fixe du ligament large s'insère sur la région sous-lombaire et les faces latérales du bassin selon une ligne caudo-ventrale oblique qui débute crânialement au niveau de l'extrémité de l'apophyse transverse de la dernière ou de l'avant dernière vertèbre lombaire et se termine sur la face interne du ligament sacro-sciatique en un point placé à mihauteur du bassin, en regard de la portion caudale de la crête sciatique.

\section{II.A.2.) BORD CRANIAL}

Le BORD CRANIAL ou base du ligament large est oblique ventralement, caudalement et un peu médialement. Il s'étend de la région sous-lombaire à l'ovaire et correspond au ligament SUSPENSEUR DE L'OVAIRE (Lig. suspensorium ovarii).

\section{II.A.3.) BORD VENTRAL}

Le BORD VENTRAL s'insère sur la moitié rostrale du vagin et sur l'utérus. Crânialement, il se fixe sur l'ovaire et porte l'oviducte par l'intermédiaire du mésosalpinx.

L'insertion du bord ventral du ligament large ne s'effectue pas de la même façon sur les différentes portions du tractus génital. Au niveau du vagin, elle se localise à la limite de la face ventrale et des bords latéraux de l'organe. Elle s'effectue ensuite sur le bord ventral (Margo mesometricus) de la portion caudale des cornes, sur la concavité de l'anse cornuale et sur le bord dorsal de la portion tubaire. L'oviducte est compris entre les lames séreuses du mésosalpinx. L'ovaire enfin est fixé au mésovarium distal par son bord dorsal et une partie plus ou moins importante de son pôle utérin et de sa face viscérale.

La portion du ligament large qui s'insère sur l'utérus et les portions voisines du vagin porte le nom de MESOMETRE (Mesometrium). Celle qui suspend l'ovaire est le MESOVARIUM (Mesovarium). En raison de la présence du MESOSALPINX, ce dernier est subdivisé en MESOVARIUM PROXIMAL (Mesovarium proximale) qui s'étend de la région sous-lombaire au mésosalpinx et en MESOVARIUM DISTAL (Mesovarium distale), très court, qui va du Mésosalpinx à l'ovaire. 
Il est important de remarquer que l'insertion utérine du ligament large n'est pas la même chez Bos taurus et chez Bos indicus. Chez le premier, elle s'effectue sur le bord ventral du corps et des cornes utérines. Chez le second, seule la portion caudale des cornes présente cette disposition. La portion crâniale est suspendue au méso par son bord dorsal tandis que la portion moyenne, celle qui subit le plus fort développement au cours de la gestation, est solidement maintenue en place par le méso qui unit les deux portions de l'anse cornuale. Il résulte de ce fait que le facteur anatomique prédisposant aux torsions de l'utérus ne peut être invoqué chez le zébu comme chez les taurins. Cet accident de la gestation semble, de fait, inconnu dans cette espèce. Une enquête menée auprès des responsables des services d'Elevage a révélé que depuis des dizaines d'années, aucun accident de ce type n'a été signalé.

\section{II.A.4.) L'ANGLE CRANIO-DORSAL}

L'ANGLE CRANIO-DORSAL DU LIGAMENT LARGE correspond à l'extrémité fixe du ligament suspenseur de l'ovaire. Il s'insère sur la région sous-lombaire au niveau du dernier espace inter-lombaire. Dans le conjonctif qui le compose circulent l'artère et la veine ovarienne, des lymphatiques et des filets nerveux appartenant au plexus ovarien.

\section{II.A.5.) L'ANGLE CRANIO-VENTRAL}

L'ANGLE CRANIO-VENTRAL DU LIGAMENT LARGE correspond à l'extrémité libre du ligament suspenseur de l'ovaire et au cul-de-sac rostral de la bourse ovarique. Il est mobile et subit des déplacements liés à ceux de l'ovaire.

\section{II.A.6.) L'ANGLE CAUDAL}

L'ANGLE CAUDAL ou SOMMET DU LIGAMENT LARGE, est tronqué. Il s'étend obliquement, médio-ventralement, des parois latérales du bassin à la portion moyenne du vagin.

\section{II.A.7.) FACE MEDIALE}

La FACE MEDIALE DU LIGAMENT LARGE est lisse. Elle forme avec le ligament opposé, la face dorsale du vagin, la face dorsale de la portion horizontale des cornes et la région sous-sacrée, une loge dans laquelle se trouve le rectum.

\section{II.A.8.) FACE LATERALE}

La FACE LATERALE DU LIGAMENT LARGE est lisse dans sa plus grande partie, mais elle présente trois formations spéciales, le mésosalpinx, le ligament rond de l'utérus et le ligament latéral de la vessie.

\section{II.A.8.a) Le mésosalpinx}

Le MESOSALPINX a été décrit à propos de l'ovaire et de l'oviducte.

\section{II.A.8.b) Le ligament rond de l'utérus}

Le LIGAMENT ROND DE L'UTERUS (Lig. teres uteri) est un cordon conjonctif, riche en fibres musculaires lisses, porté par un court méso sur la face externe du ligament large. Il commence un peu au-dessus et en arrière de l'extrémité utérine de l'ovaire par un court appendice en forme de caroncule. Il se dirige dorso-crânialement et un peu latéralement vers l'angle crânio-dorsal du ligament large et se prolonge ensuite dans le conjonctif sous-péritonéal jusqu'au voisinage de l'anneau inguinal profond. Embryologiquement, il correspond au ligament caudal du mésonéphros qui chez le mâle est une portion du gubernaculum testis et porte le nom de LIGAMENT INGUINAL ou LIGAMENT CAUDAL DE L'EPIDIDYME (Lig. caudae epididymis).

\section{II.A.8.c) Le ligament latéral de la vessie}


Le LIGAMENT LATERAL DE LA VESSIE (Lig. vesicae laterale) se détache des parties déclives de la face latérale du mésométrium et rejoint les faces latérales de la vessie.

\section{I.A.9.) STRUCTURE DU LIGAMENT LARGE}

Structuralement, le ligament large est constitué par une lame conjonctive épaisse et riche en fibres musculaires lisses au sein de laquelle circulent les vaisseaux, les nerfs ovariens et l'urètre. Il est tapissé sur ses deux faces par la séreuse péritonéale.

\section{II.B.) Topographie et rapports de l'utérus}

La situation et les rapports de l'utérus de Bos indicus varient selon qu'on observe l'organe chez la génisse, la vache gestante ou la femelle multipare.

\section{II.B.1.) CHEZ LA GENISSE}

Chez la génisse, le tractus génital est tout entier contenu dans la cavité pelvienne. Soutenu par les ligaments larges, l'utérus est situé entre le rectum et la vessie. Les portions les plus crâniales des comes prennent avec les viscères digestifs des rapports que nous avons déjà précisés à propos de l'ovaire.

\section{II.B.2.) CHEZ LA VACHE GESTANTE}

Au cours de la gestation, la come gravide se développe principalement dans sa région moyenne où s'effectue la fixation de l'œuf. Entraînée par le poids du foetus et grâce à la laxité du ligament large, cette portion de la corne subit une migration ventro-crâniale qui l'amène au bord caudal du grand épiploon qui sépare la cavité abdominale en loges sus et sous-omentales.

Comme chez Bos taurus, la corne gravide de Bos indicus s'engage le plus souvent dans la LOGE SUS-OMENTALE où elle poursuit son développement en refoulant rostralement et dorsalement la masse intestinale. Rarement elle se développe dans la LOGE SOUS-OMENTALE, entre la paroi abdominale droite et l'épiploon. Nous n'avons jamais observé la modalité signalée chez les taurins selon laquelle la corne gravide se développe à gauche, entre le fuyant du flanc et le rumen.

Parallèlement à cette migration abdominale de la portion moyenne de la corne gravide, l'extrémité caudale de l'utérus et le col utérin se rapprochent du bord crânial du pubis qu'ils atteignent vers le $6^{\mathrm{e}}$ mois chez les primipares et dépassent ohez les multipares tandis que l'extrémité crâniale de la corne subit un déplacement parallèle à celui de l'ovaire.

\section{II.B.3.) CHEZ LA VACHE MULTIPARE}

Après la mise-bas, l'utérus et le ligament large subissent une rétraction qui tend mais ne parvient jamais à les ramener à leurs dimensions primitives, si bien qu'après chaque gestation l'utérus est un peu plus gros et en position un peu plus crâniale et ventrale que dans l'intervalle-vêlage précédent.

Chez la vache multipare, l'utérus peut de ce fait être tout entier en position abdominale. Le vagin, qui lui aussi a subi l'action de la gravidité, occupe alors toute la longueur de la cavité pelvienne.

Comme pour la migration ovarienne, cette migration utérine semble plus importante chez Bos taurus que chez Bos indicus en raison de la précocité de la puberté et du faible intervalle qui sépare deux vêlages consécutifs dans cette espèce.

\section{STRUCTURE}

La paroi de l'utérus est composée de trois couches: la tunique muqueuse, la tunique 
musculeuse et la tunique séreuse. Les deux premières subissent des modifications progressives avec l'âge et des remaniements périodiques avec le cycle œstral. Pendant la gestation, elles s'adaptent aux fonctions de protection, de nutrition et d'expulsion du fætus.

\section{III.A.) La tunique séreuse}

La TUNIQUE SEREUSE ou PERIMETRE (Tunica serosa; Perimetrium) est formée d'une lame mince de cellules aplaties, la LAME SEREUSE EPITHELIALE (Lamina epitheliale serosae; Mesothelium), qui se réfléchit sur les faces des ligaments larges et d'une LAME SOUSSEREUSE (Lamina subserosa; Lamina propria serosae) conjonctivo-élastique qui adhère fortement à la couche superticielle de la tunique musculaire et se prolonge dans les ligaments larges en formant le PARAMETRE (Parametrium). Lame sous-séreuse et paramètre livrent passage à des nerfs et à de nombreux et flexueux vaisseaux utérins.

\section{III.B.) La tunique musculeuse}

La TUNIQUE MUSCULEUSE ou MYOMETRE (Tunica muscularis; Myometrium) est formée de fibres musculaires lisses groupées en faisceaux et séparées les unes des autres par des lames conjonctivo-élastiques anastomosées.

L'orientation des faisceaux musculaires ainsi que l'importance et la nature des lames conjonctives de soutien permettent de distinguer trois couches dans le myomètre.

\section{III.B.1.) COUCHE SUPERFICIELLE DU MYOMETRE}

La COUCHE SUPERFICIELLE (Stratum longitudinale externum) est formée de faisceaux musculaires à direction longitudinale prédominante. Ces faisceaux délèguent dans la lame sousséreuse et dans le paramètre des prolongements qui portent le nom de MUSCULAIRE DE LA SEREUSE (Muscularis serosae).

Les lames conjonctivo-élastiques qui séparent les faisceaux musculaires de la couche superficielle sont d'importance moyenne. Elles sont à peine moins abondantes que les faisceaux musculaires sur la face dorsale des cornes et plus rares au fur et à mesure qu'on se rapproche de la face ventrale.

\section{III.B.2.) COUCHE MOYENNE DU MYOMETRE}

La COUCHE MOYENNE ou COUCHE VASCULAIRE (Stratum vasculare) est caractérisée par un important réseau vasculaire plexiforme dont les mailles se répartissent au sein d'un tissu conjonctivo-élastique et musculaire.

Les faisceaux musculaires de cette couche s'entrecroisent en tous sens pour former un nouveau réseau à larges mailles qui se superpose au réseau vasculaire. Cette couche semble jouer un rôle important dans l'irrigation de la paroi utérine. En comprimant les gros vaisseaux veineux, elle serait, en particulier, capable de provoquer l'hyperhémie de la muqueuse utérine par bloquage partiel de la circulation de retour.

Les lames conjonctivo-élastiques intriquées les unes dans les autres sont très abondantes dans la couche moyenne. Elles occupent tout l'espace laissé libre par les réseaux vasculaire et musculaire.

\section{III.B.3.) COUCHE PROFONDE DU MYOMETRE}

La COUCHE PROFONDE du Myomètre (Stratum circulare internum) est très riche en fibres musculaires. A son niveau, la trame conjonctivo-élastique est discrète et le réseau vasculaire réduit à quelques vaisseaux de petit calibre.

Les fibres musculaires, puissantes et tassées les unes contre les autres se disposent en 
faisceaux à orientations transversales ou circulaires prédominantes. A la jonction des deux cornes, les fibres musculaires de cette couche s'adossent les unes contre les autres pour former l'éperon utérin.

\section{III.B.4.) VARIATIONS SUBIES PAR LA TUNIQUE MUSCULEUSE}

La tunique musculeuse de l'utérus subit des variations selon la région observée, l'âge, le cycle cestral et la gestation.

\section{III.B.4.a) Variations topographiques}

PRES DE LA JONCTION TUbo-UTERINE la tunique musculeuse s'amincit. Cette réduction porte principalement sur la couche vasculaire qui se réduit à une mince lame qui sépare les plans musculaires longitudinal et circulaire dans l'oviducte.

AU NIVEAU DU COL UTERIN, les trois couches de la tunique musculeuse de l'utérus subissent d'importantes modifications.

La couche moyenne perd ses plus volumineux vaisseaux et se transforme en une lame conjonctivo-musculaire très riche en fibres élastiques qui donne sa consistance ferme au col.

La couche externe associée aux éléments musculaires de la Muscularis serosae se prolonge dans le col. Elle perd sa disposition longitudinale et prend une orientation oblique, puis spiralée.

Dans la région cervicale, la couche interne est la plus épaisse. Constituée de faisceaux musculaires à disposition oblique et circulaire, elle se subdivise en 3 ou 4 couches concentriques, séparées par des lames conjonctives discrètes et mobiles l'une par rapport à l'autre.

\section{III.B.4.b) Variations avec l'âge et l'état fonctionnel}

A LA NAISSANCE, la paroi utérine est peu épaisse en raison du faible développement des couches profonde et moyenne de son myomètre.

AVANT LA PUBERTE, à chaque poussée folliculaire avortée, la couche musculeuse subit une double évolution qui se traduit par l'hyperplasie et l'hypertrophie de ses éléments constituants. L'hyperplasie correspond à une augmentation du nombre des cellules musculaires de la couche profonde et à l'accroissement du nombre des vaisseaux et des fibres de la couche moyenne. L'hypertrophie est modérée. Elle porte essentiellement sur la dimension des fibres musculaires et sur le diamètre des vaisseaux.

Ces poussées évolutives successives se traduisent par l'augmentation progressive de l'êpaisseur de la paroi utérine et par la mise en place, au moment de la puberté, d'un myomètre normal, considérablement plus développé que celui de la génisse à la naissance.

APRES LA PUBERTE, la couche musculeuse subit à chaque cycle des modifications qui intéressent les fibres musculaires et le réseau vasculaire de la couche moyenne.

Pendant la période ostrogénique du cycle, les cellules musculaires augmentent de volume. Elles atteignent leur dimension maximale au moment de l'œstrus. A cette période et pendant les jours qui suivent, elles sont en outre éminemment contractiles. Cette hyperactivité myométriale jointe à l'hypersécrétion muqueuse qui accompagne l'œstrus est évoquée par de nombreux auteurs à propos de la résistance aux infections utérines que l'on constate au moment des chaleurs chez un grand nombre de femelles.

Le réseau vasculaire de la couche moyenne s'hypertrophie au cours de la phase ostrogénique du cycle. Il atteint son développement maximal au moment de l'œstrus. A ce moment, les fibres musculaires qui lui sont associées sont aussi hyper-contractiles, si bien qu'à la suite d'un bloquage partiel de la circulation de retour, l'hyperhémie de la muqueuse utérine se produit. 
Au cours de la seconde moitié du post-œstrus, les phénomènes précédemment décrits régressent. La tunique musculaire prend un état de repos jusqu'au début du pro-œstrus suivant.

AU COURS DE LA GESTATION, les modifications portent principalement sur les couches moyennes et profondes. La couche moyenne augmente d'épaisseur et enrichit son réseau vasculaire, tandis que les myocytes de la couche profonde s'hyperplasient et s'hypertrophient pour atteindre leur développement maximal au moment du part. Pendant toute la durée de la gestation, les myocytes de la couche moyenne règlent le débit sanguin dans les parois utérines, jouant le rôle d'un véritable « cour utérin » par contraction (systole) et relâchement (diastole) des fibrilles qui les composent.

\section{III.C.) La tunique muqueuse}

La TUNIQUE MUQUEUSE ou ENDOMETRE (Tunica mucosa; Endometrium) est formée d'un épithélium et d'un chorion qui renferme des glandes.

\section{III.C.1.) LA LAME MUQUEUSE EPITHELIALE}

La LAME MUQUEUSE EPITHELIALE (Lamina epithelialis mucosae) est simple, prismatique ou cylindrique et composée de deux types cellulaires : des CELLULES CILIEES (Epitheliocytus ciliatus) à noyau ovalaire dont l'apex est garni de kinocils et des CELLULES NON CILIEES (Epitheliocytus nonciliatus) à noyau allongé, et d'aspect souvent sécrétoire. Des CELLULES INTERCALAIRES étroites, qui correspondent à des éléments sénescents en voie d'expulsion, peuvent aussi être observées.

\section{III.C.2.) LE CHORION DE LA MUQUEUSE}

Le chorion ou Lamina propria est épais. Il est formé d'un tissu conjonctif réticulé (Stroma endometrium), d'autant plus riche en cellules qu'on se rapproche de l'épithélium. Sa couche la plus profonde renferme de nombreux vaisseaux sanguins et lymphatiques.

\section{II.C.3.) LES GLANDES UTERINES}

Le chorion renferme des glandes en tube, parfois simples et le plus souvent ramifiées à leurs extrémités : LES GLANDES UTERINES (Glandulae uterinae). Ces glandes débouchent dans les vallées inter-caronculaires de la surface de la muqueuse, isolément ou par groupe de 3 ou 4, au fond de dépressions: les CRYPTES UTERINS (Cryptae uterinae). Comme l'épithélium utérin, l'épithélium glandulaire est composé de cellules ciliées très peu nombreuses, de cellules glandulaires et de cellules intercalaires.

\section{III.C.4.) VARIATIONS SUBIES PAR LA TUNIQUE MUQUEUSE}

La tunique muqueuse présente des variations selon la zone utérine considérée, l'âge, le cycle cestral et l'état de gestation.

\section{III.C.4.a) Variations topographiques}

D'importantes modifications de la muqueuse peuvent être observées aux niveaux des caroncules, de la jonction tubo-utérine et du canal cervical.

Les CARONCULES (Carunculae) sont des formations en relief dont la surface est parsemée de dépressions infundibulaires ou CRYPTES CARONCULAIRES (Cryptae carunculae) dans lesquels se fixent les villosités choriales au cours de la gestation.

Elles sont constituées d'un relèvement du chorion qui perd ses glandes et s'épanouit pour former le berceau conjonctivo-vasculaire de la caroncule.

L'épithélium qui tapisse cette formation diffère de celui du reste de la paroi utérine. Il est 

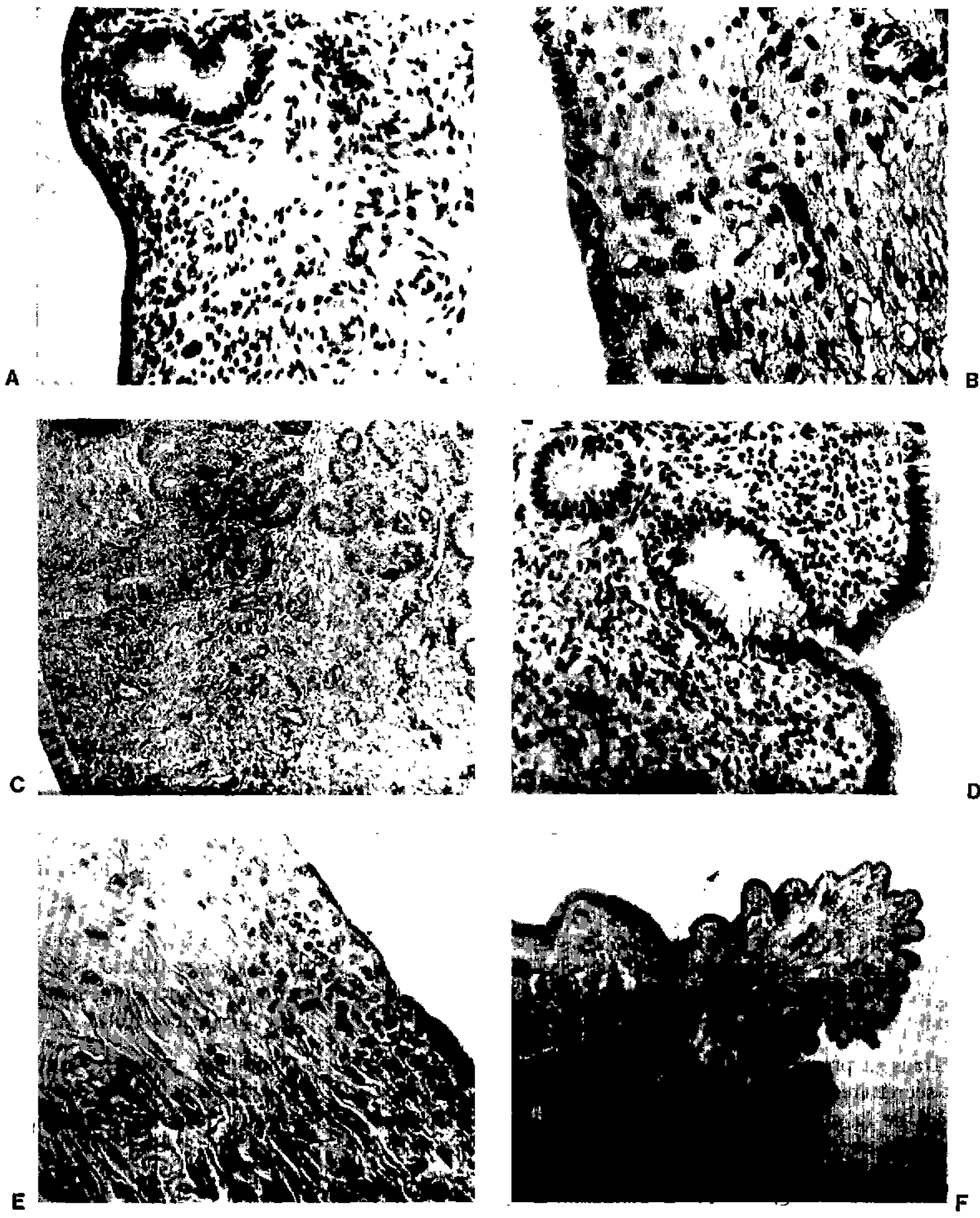

Fig. 11. - Structure de l'utérus, du vagin et de la vulve de la femelle zébu.

A et B): Muqueuse utérine pendant le di-cstrus (A: $\times 250 ; \mathrm{B}: \times 400$ ).

$C$ et $D):$ Muqueuse utérine en début de la phase progestative du cycle $(C: \times 100 ; D: \times 250)$.

E): Muqueuse vaginale $(X 400)$.

F) : Lèvre vulvaire (en haut et à gauche, muqueuse; à droite, revêtement cutané) ( $X 12)$. 
cubique simple et composé de cellules dépourvues de kynocils et sans caractère sécrétoire.

- AU NIVEAU DE LA JONCTION TUBO-UTERINE, la transition entre muqueuse utérine et muqueuse tubaire s'effectue rapidement. Les cellules de l'épithélium utérin changent de type. Le chorion perd ses glandes et s'amincit.

- AU NIVEAU DU CANAL CERVICAL, les cellules de l'épithélium, de type muqueux prédominant (Mucocytus) sont basses. Le chorion, très épais et moins riche en cellules, accroît son contingent de fibres élastiques. Il perd ses glandes et est parcouru par un abondant et fin réseau vasculaire.

\section{III.C.4.b) Variations liées à l'âge et à l'état fonctionnel}

AVANT LA PUBERTE, la muqueuse utérine est à l'état de repos permanent. L'épithélium superficiel et celui des glandes est formé de cellules cubiques ou prismatiques basses et dépourvues de kinocils qui ne présentent que sporadiquement des signes sécrétoires discrets. Pendant cette période, le chorion reste mince. Il est en outre pauvre en fibres élastiques et dépourvu de glandes.

PEU AVANT LA PUBERTE, l'épithélium se différencie par apparition des divers types cellulaires. Le chorion s'épaissit, il se garnit de glandes et s'enrichit en fibres élastiques.

AVEC LES PREMIERES CHALEURS une série de transformations cycliques s'installent qui, bien que parfois interrompues par des périodes d'anœstrus, se prolongeront pendant toute la durée de la vie sexuelle. Ces modifications intéressent le chorion, les glandes utérines et l'épithélium utérin.

LE CHORION est très réduit vers le milieu du di-cstrus. En raison de l'allongement et de la prolifération des glandes utérines qu'il contient, il s'épaissit progressivement pendant toute la phase cstrogénique du cycle pour atteindre son développement maximal au moment de l'ostrus. Au cours de la phase progestative, il diminue progressivement pour atteindre son épaisseur la plus faible vers le milieu du di-cestrus suivant.

LES GLANDES UTERINES sont courtes, peu ramifiées et peu circonvolutionnées à la fin de la période progestative. A ce moment, leur épithélium est bas, formé de cellules presque cubiques, dépourvues d'activité sécrétoire et à noyaux alignés près de la vitrée.

Pendant la période œstrogénique, les glandes s'allongent, prolifèrent et se circonvolutionnent. Histologiquement, elles transforment le chorion qui s'épaissit pendant qu'elles se développent en une véritable « dentelle utérine ». En même temps, les cellules glandulaires deviennent plus hautes. Certains noyaux s'éloignent de la basale et l'épithélium prend un aspect "pseudo-stratifié ».

Dans la première moitié de la phase progestative, l'épithélium glandulaire prend les caractères sécrétoires très nets. Des produits s'accumulent à l'apex cellulaire. Ils sont rejetés dans la lumière de la glande vers la fin du post-œstrus ou au début du diœstrus. Puis les glandes se simplifient, se raccourcissent et entrent en phase de repos.

L'EPITHELIUM UTERIN est formé de cellules au repos à la fin du post-œstrus. A cette période et pendant la première moitié du di-cestrus, la majorité des noyaux sont alignés près de la basale. Dès le début de la phase estrogénique, les cellules prennent une activité sécrétoire. Elles augmentent de volume. Les produits de sécrétion accumulés d'abord dans la région infranucléaire, puis vers l'apex, déplacent les noyaux qui occupent des niveaux différents d'une cellule à l'autre et donnent à l'épithélium l'aspect «pseudo-stratifié ». La sécrétion épithéliale de surface se produit au moment de l'œstrus et dans les jours qui suivent. Puis, au cours de la période progestative, les cellules présentent peu à peu des images de repos.

Au cours de l'œstrus et du post-œstrus, l'utérus est donc le siège d'une double sécrétion, à la fois de surface et glandulaire. Ces sécrétions joueraient un rôle important dans la capacitation du spermatozoïde lors de sa migration dans les voies génitales femelles. Notons que c'est pen- 
dans ces mêmes périodes œstrale et post-œstrale que le réseau vasculaire de la couche moyenne du myomètre provoque une hyperhémie de la muqueuse.

AU DEBUT DE LA GESTATION, une abondante sécrétion glandulaire et épithéliale se produit. Le phénomène coïncide avec la phase de migration de l'œuf et celle de la PLACENTATION DIFFUSE, transitoire du chorion embryonnaire.

Plus tard, la PLACENTATION devient COTYLEDONAIRE et épithélio-choriale. La nutrition embryonnaire s'effectue à partir du sang maternel. L'activité glandulaire de la muqueuse diminue.

APRES LA MISE BAS, les villosités choriales se désengrènent. La muqueuse et la musculeuse utérines, jusque là considérablement hypertrophiées, involuent et reprennent des caractères structuraux normaux.

CHEZ LES SUJETS AGES enfin, l'endomètre s'atrophie. Les glandes disparaissent et le chorion s'amincit. Les cellules épithéliales gardent en permanence leur aspect de repos.

\section{IRRIGATION ET INNERVATION DE L'UTERUS}

En raison de ses fonctions, l'utérus est très richement irrigué et innervé. Son réseau vasculaire, en particulier, est disposé de telle façon qu'il se prête facilement aux variations d'activité et de volume que subit l'organe pendant le cycle ostral et la gestation.

\section{IV.A.) Les artères de l'utérus}

Contrairement à ce qu'on observe chez les autres ongulés, l'utérus du zébu reçoit son irrigation artérielle d'une seule source: l'artère utérine. Dans cette espèce, en effet, le rameau utérin de l'artère ovarienne (ou artère tubo-utérine) ne se distribue qu'à l'oviducte et n'étend pas son territoire d'irrigation au-delà de la jonction tubo-utérine. De même, le rameau utérin de l'artère vaginale (ou artère utérine caudale des anglo-saxons ou artère cervico-utérine des auteurs français) n'atteint jamais l'extrémité caudale de l'utérus. L'interférence du vagin sur le col isole ce dernier du territoire d'irrigation de l'artère vaginale.

L'ARTERE UTERINE ( $A$. uterina) est une branche collatérale de l'artère ombilicale (A. umbilicalis). Elle se détache à $1 \mathrm{~cm}-1 \mathrm{~cm} 1 / 2$ de l'origine de ce vaisseau, s'engage dans le ligament large selon un trajet caudo-ventral sinueux et se divise de façon variable suivant les sujets en trois rameaux, tous destinés à l'utérus.

\section{IV.A.1.) LE RAMEAU CRANIAL}

Le RAMEAU CRANIAL de l'artère utérine irrigue la portion crâniale ou tubaire des cornes par deux branches principales réunies entre elles par une anastomose. La branche rostrale s'anastomose avec le rameau utérin de l'artère ovarienne. La branche aborale échange avec la branche rostrale du rameau moyen plusieurs collatérales d'union.

\section{IV.A.2.) LE RAMEAU MOYEN}

LE RAMEAU MOYEN est le plus volumineux des trois. Il se subdivise généralement en trois branches.

Sa branche rostrale irrigue la portion moyenne de la corne utérine (ou anse cornuale). Ses branches moyennes et caudales se distribuent à la portion rectiligne des cornes.

Les trois branches du rameau moyen sont unies entre elles par des anastomoses qui s'effectuent toutes au contact ou dans l'épaisseur de la paroi utérine. Celles qui se localisent à distance du tractus génital sont très rares. Lorsqu'elles existent, elles sont toujours très grêles.

La branche caudale du rameau moyen s’unit au rameau caudal de l'artère utérine. Sa branche rostrale s'anastomose avec le rameau crânial. 


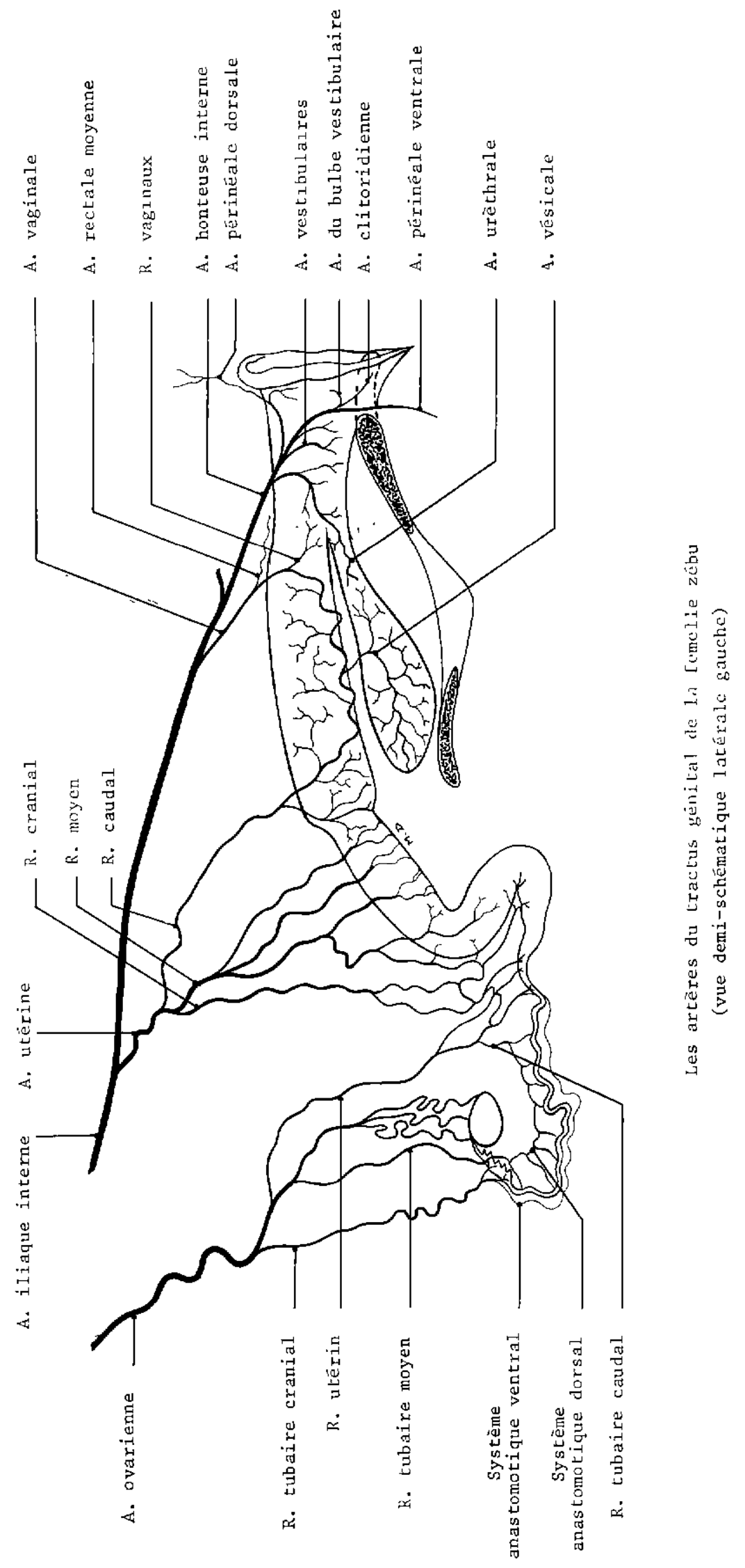






Les veines du tractus génital de la femelle zêbu (vue demi-schêmatique latérale gauche) 


\section{IV.A.3.) LE RAMEAU CAUDAL}

Le RAMEAU CAUDAL de l'artère utérine est très oblique caudo-ventralement. Au niveau du bourrelet fornical, il se divise en deux branches. Une branche rostrale qui irrigue le col utérin, et une branche caudale qui longe la face latérale du vagin et s'anastomose à plein canal avec l'artère vaginale.

\section{IV.B.) Les veines de l'utérus}

L'utérus et la portion crâniale du vagin de la femelle zébu sont drainés par deux volumineuses veines marginales, situées de part et d'autre du plan médian, sur les faces latérales du tractus génital. Le sang de ces vaisseaux est recueilli par un système veineux satellite du système artériel, c'est-à-dire, en avant par la veine ovarienne, en région moyenne par la veine utérine et caudalement par la veine vaginale. De ces trois voies, la moyenne est la plus grêle. Le drainage sanguin de l'utérus s'effectue donc principalement par les voies extrêmes: la veine ovarienne et la veine vaginale.

Sur certains sujets, on peut observer en outre un quatrième vaisseau collecteur de la veine marginale qui unit la portion caudale de ce vaisseau à la veine iliaque interne. Son trajet oblique dorso-crânialement et satellite de l'uretère permet de l'assimiler à la veine uretérale moyenne.

\section{IV.B.1.) LA VEINE MARGINALE DE L'UTERUS}

LA VEINE MARGINALE DE L'UTERUS draine la matrice et la portion crâniale du vagin. Elle a un aspect variable selon l'âge et le territoire envisagé.

Simple et presque rectiligne avant la puberté, elle devient polymorphe au cours de la première gestation et peut dès lors être divisée caudo-rostralement en 4 portions : une portion sinueuse, une portion plexiforme, une portion en anse et une portion rectiligne.

\section{IV.B.1.a) La portion sinueuse de la veine marginale de l'utérus}

LA PORTION SINUEUSE DE LA VEINE MARGINALE DE L'UTERUS est formée d'une volumineuse veine qui décrit des sinuosités sur la paroi latérale de la moitié rostrale du vagin. Elle draine la portion correspondante de l'organe et reçoit des affluents vaginaux par sa face profonde et ses bords dorsal et ventral.

Caudalement, la portion sinueuse de la veine marginale se prolonge par la veine vaginale. Crânialement, elle s'unit à la portion plexiforme. Dorsalement, elle est drainée de manière inconstante par la veine uretérale moyenne.

\section{IV.B.1.b) La portion plexiforme de la veine marginale de l'utérus}

LA PORTION PLEXIFORME DE LA VEINE MARGINALE DE L'UTERUS est formée de 2 ou 3 vaisseaux anastomosés entre eux. Elle draine la portion caudale des cornes et le col utérin. Elle s'unit en arrière à la portion sinueuse et crânialement rejoint la branche caudale de la portion en anse. Dorsalement, elle se prolonge par les racines de la veine utérine.

\section{IV.B.1.c) La portion en anse de la veine marginale de l'utérus}

LA PORTION EN ANSE DE LA VEINE MARGINALE DE L'UTERUS draine la partie moyenne des cornes (ou anse cornuale) dont elle épouse la forme. Elle est constituée par deux branches crâniale et caudale qui s'anastomosent à plein caudal dans la concavité de l'anse et s'unissent à l'autre extrémité pour former la portion rectiligne de la veine marginale.

La branche caudale de la portion en anse est unie à l'extrémité orale de la portion plexiforme. La branche crâniale s'anastomose à la racine aborale de la veine tubo-utérine. 


\section{IV.B.1.d) La portion rectiligne de la veine marginale}

LA PORTION RECTILIGNE DE LA VEINE MARGINALE prolonge crânio-dorsalement la portion en anse. Dans l'épaisseur du ligament large, elle suit un trajet parallèle à celui du rameau utérin de l'artère ovarienne et se termine dans la veine ovarienne, dont elle est un affluent caudal. Près de son origine, la portion rectiligne de la veine marginale reçoit un affluent crânial : la veine tubo-utérine.

\section{IV.B.2.) LA VEINE TUBO-UTERINE}

LA VEINE TUBO-UTERINE, grêle et courte, résulte de la réunion de deux branches :

- La branche crâniale correspond à la veine tubaire caudale. Elle a déjà été étudiée à propos de l'oviducte;

- La branche caudale draine la portion tubaire des cornes dont elle longe le bord dorsal. Caudalement, elle s'anastomose avec la branche crâniale de la portion en anse.

Après un court trajet, la veine tubo-utérine rejoint la veine marginale de l'utérus à laquelle elle s'unit dans la première partie de sa portion rectiligne.

\section{IV.B.3.) LA VEINE OVARIENNE}

LA VEINE OVARIENNE ( $V$. ovarica) a été étudiée. Elle reçoit sur son bord caudal le volumineux affluent qu'est la veine marginale et rostralement la veine tubaire crâniale. Elle représente l'un des deux éléments majeurs du drainage de l'utérus.

\section{IV.B.4.) LA VEINE UTERINE}

LA VEINE UTERINE ( $V$, uterina) est très grêle. Satellite de l'artère correspondante, elle se constitue de manière variable, par l'union de trois ou quatre racines qui naissent du bord dorsal de la portion plexiforme de la veine marginale. Après un court trajet oblique ventro-dorsal la veine utérine se jette dans la veine honteuse interne.

\section{IV.B.5.) LA VEINE URETERALE MOYENNE}

LA VEINE URETERALE MOYENNE est inconstante. Elle naît sur la portion caudale de la veine marginale de l'utérus, se dirige crânio-dorsalement dans le ligament large et rejoint l'uretère qu'elle longe sur une partie de son trajet. Elle se termine dans la veine iliaque interne et reçoit des affluents uretéraux.

\section{IV.B.6.) LA VEINE VAGINALE}

La VEINE VAGINALE sera étudiée avec la portion copulatrice du tractus génital.

\section{IV.C.) Les lymphatiques utérins}

Les vaisseaux lymphatiques sont très nombreux dans la paroi utérine. Ils y forment des réseaux importants dans le chorion de la muqueuse, dans la couche moyenne de la musculeuse, et dans la lame conjonctive sous-séreuse.

Le drainage de ces réseaux est assuré par des vaisseaux lymphatiques qui sont satellites des vaisseaux sanguins. Ils rejoignent le groupe des GANGLIONS ILIAQUES MEDIAUX (Lymphodoni iliaci mediales).

\section{IV.D.) Les neris de l'utérus}

Les nerfs de l'utérus proviennent du plexus hypogastrique. Ils cheminent avec les vaisseaux et se distribuent à la musculeuse, aux vaisseaux et aux glandes. Certains filets atteignent la face profonde de l'épithélium ovarien. 


\section{Chapitre III}

\section{La portion copulatrice des organes génitaux de la femelle Zébu}

La section copulatrice de l'appareil génital femelle est un conduit musculo-membraneux qui reçoit l'organe copulateur du mâle au moment du coït et livre passage au fœetus lors de la mise bas. Dans ses régions les plus caudales, il constitue en outre une portion commune aux voies génitale et urinaire.

Il est formé de trois segments qui se succèdent crânio-caudalement : le vagin, le vestibule vaginal et la vulve.

Dans un premier temps, nous étudierons la morphologie et la structure de chacune de ces trois portions. Nous traiterons ensuite des moyens de fixité et de la vasculo-innervation de l'ensemble.

\section{MORPHOLOGIE ET STRUCTURE DE LA PORTION COPULATRICE DU TRACTUS GENITAL DE LA FEMELLE ZEBU}

\section{I.A.) Le vagin}

Le VAGIN (Vagina) communique crânialement avec la cavité de l'utérus par l'intermédiaire du col utérin. En arrière, l’hymen le sépare du vestibule vaginal.

\section{I.A.1.) CONFORMATION EXTERIEURE ET RAPPORTS}

Le vagin est de consistance molle. En raison de la compression qu'il subit de la part du rectum, il est aplati dorso-ventralement. Sa paroi dorsale (Paries dorsalis) se moule sur la face ventrale du rectum, tandis que sa paroi ventrale (Paries ventralis) répond à l'urèthre, au col de la vessie et au plancher de la cavité pelvienne.

Ses bords latéraux sont en rapport avec les parois du bassin. Dans leur portion la plus crâniale, ils sont croisés obliquement par les uretères qui cheminent caudo-ventralement dans le ligament large et les ligaments latéraux de la vessie.

Crânialement le vagin recouvre plus ou moins complètement le col utérin sur lequel jl interfère en formant un bourrelet qui, par sa forme circulaire, pourrait être appelé BOURRELET VAGINAL ou COURONNE VAGINALE (Corona vaginalis).

Caudalement, aucune démarcation nette ne le sépare du vestibule vaginal.

Les dimensions du vagin sont très variables selon l'âge et l'état fonctionnel du tractus génital du sujet. Court ( 4 à $10 \mathrm{~cm}$ ) et étroit ( 3 à $4 \mathrm{~cm}$ de diamètre en moyenne) chez la génisse, il peut atteindre chez la vache multipare une longueur de 20 à $25 \mathrm{~cm}$, pour un diamètre transversal de 8 à $10 \mathrm{~cm}$ et un diamètre dorso-ventral de 5 à $6 \mathrm{~cm}$.

Au moment du part, le vagin est très dilaté. Il occupe toute la cavité pelvienne en refoulant dorsalement et ventralement le rectum et la vessie vidés de leur contenu.

\section{I.A.2.) CONFORMATION HNTERIEURE}

En place et à l'état de repos, les parois dorsale et ventrale du vagin sont appliquées l'une contre l'autre: la cavité vaginale est virtuelle. 
Après prélèvement du tractus génital et ouverture du plafond de la section copulatrice, la muqueuse peut être observée. Elle est formée de deux portions d'à peu près égale longueur.

La portion crâniale du vagin est revêtue d'une muqueuse rosée, plus ou moins rougeâtre qui forme une série de 5 à 6 BOURRELETS VAGINAUX (Rugae vaginales), circulaires et transversaux, séparés par des vallées étroites. Rostralement, cette muqueuse d'aspect plissé se réfléchit sur le col utérin en formant un cul-de-sac, le FORNIX DU VAGIN (Fornix vaginae) qui, le plus souvent, interfère sur toute la longueur du col. Les bourrelets circulaires de la paroi vaginale se réfléchissent sur le col et garnissent son revers externe de 4 ou 5 reliefs muqueux annulaires.

La portion caudale du vagin est revêtue d'une muqueuse lisse. Elle est séparée du vestibule vaginal par un hymen (Hymen), toujours peu développé, qui marque l'emplacement de l'ORIFICE VAGINAL (Ostium vaginae).

Les parois du vagin sont normalement recouvertes de mucus. Au moment de l'œstrus, ce mucus est particulièrement abondant et la muqueuse qui le sécrète est congestionnée. Au cours du stade progestatif du cycle, la muqueuse reprend peu à peu la couleur rosée qui lui est habituelle, le mucus qui la recouvre se raréfie et devient plus épais. Au cours de la gestation, la muqueuse est terne et sèche, de coloration rose très pâle avec des reflets jaunâtres.

\section{I.A.3.) STRUCTURE}

La paroi du vagin est structuralement composée de trois couches. L'externe est, suivant la région, une séreuse ou une adventice. La couche moyenne est une musculeuse, la couche interne une muqueuse.

La TUNIQUE ADVENTICE (Tunica adventicia) est formée de tissu conjonctif riche en fibres élastiques et en lobules adipeux. Ses couches les plus profondes se disposent en lames concentriques à la périphérie de la tunique musculeuse. Dans l'espace rétro-péritonéal (Spatium retroperitoneale) ses couches les plus superficielles se confondent avec le conjonotif ambiant.

Crânialement, dans la zone du tractus génital qui est recouverte par le péritoine, la tunique adventice se transforme en une LAME SOUS-SEREUSE (Tela subserosa) lâche que recouvre la LAME SEREUSE EPITHELIALE (Lamina epithelialis serosea) pour former la TUNIQUE SEREUSE (Tunica serosa).

LA TUNIQUE MUSCULEUSE (Tunica muscularis) est mince. Elle est formée de fibres musculaires lisses disposées en faisceaux obliques et entrecroisés qui tendent à prendre une disposition circulaire dans la profondeur. Les faisceaux musculaires sont séparés par un tissu conjonctif abondant dans lequel circulent de nombreux vaisseaux.

LA TUNIQUE MUQUEUSE (Tunica mucosa) est formée d'un épithélium (Lamina epithelialis mucosae) qui repose sur un chorion conjonctivo-élastique (Lamina propria).

L'EPITHELIUM (Epithelium stratificatum non cornescens) est stratifié, pavimenteux et non corné. Les cellules qui le constituent se disposent en 3 couches.

La couche basale (Stratum basale) est formée de petites cellules ( $15 \mu$ en moyenne) rondes ou ovalaires, à noyaux volumineux. Elles sont douées d'une grande activité mitotique et représentent la couche génératrice de l'épithélium.

La couche intermédiaire (Stratum intermedium) est formée de cellules plus volumineuses, polygonales ou polyédriques.

Dans la couche superficielle (Stratum superficiale) les cellules s'aplatissent, subissent un début de kératinisation puis desquament dans la lumière de la cavité vaginale. Cette couche renferme aussi des cellules à mucus, nombreuses dans la portion crâniale du vagin et rares dans sa portion caudale.

La lame épithéliale de la muqueuse subit des modifications au cours du cycle estral. 
Pendant la phase œstrogénique, les cellules à mucus augmentent en nombre. Au moment de l'œstrus, elles sécrètent un mucus clair, abondant et fluide qui sèche et se cristallise en formant des images disposées en feuille de fougère (images ptéridiennes) lorsqu'il est recueilli par frottis sur une lame. Dans la seconde moitié du post-cestrus, l'activité des cellules mucipares diminue progressivement. Le mucus devient moins abondant. II se densifie et prend un aspect grumeleux. Au cours du di-cestrus, un grand nombre de cellules mucipares sont éliminées et rejetées dans la cavité vaginale.

Les cellules plates de la couche superficielle de la muqueuse augmentent en nombre pendant la phase œstrogénique du cycle. Au cours de cette période, elle se kératinisent et perdent consécutivement leur affinité pour les colorants basiques; elles deviennent « acidophiles». Pendant le post-œstrus les cellules acidophiles desquament ou sont phagocytées par des leucocytes d'origine sanguine qui envahissent la tunique muqueuse. Leur fonction phagocytaire accomplie, ces leucocytes chargés de débris cellulaires sont éliminés dans la cavité vaginale et se mêlent au mucus auquel ils donnent un aspect floconneux caractéristique. Pendant le di-œstrus, l'épithélium s'amincit. La reprise d'activité de la couche génératrice basale marque le début du pro-œstrus.

Ces transformations cycliques de l'épithélium vaginal sont provoquées par les hormones sexuelles dont l'activité peut consécutivement être contrôlée par l'examen du mucus et la détermination des populations cellulaires recueillies par frottis vaginaux. Cette méthode de diagnostic hormonal donne d'excellents résultats dans certaines espèces. Chez les bovins, elle est difficilement utilisable en raison des faibles différences qu'on observe d'une période à l'autre du cycle, de l'importance des variations individuelles et des interférences cytologiques fréquentes qui résultent de la cytolyse et des processus infectieux locaux.

\section{I.B.) Le vestibule vaginal}

Le VESTIBULE VAGINAL (Vestibulum vaginae) est un conduit commun aux voies urinaire et génitale. Il prolonge caudalement le vagin et reçoit à son extrémité crâniale le conduit uréthral. Caudalement, il communique avec l'extérieur par la vulve.

\section{I.B.1.) CONFORMATION EXTERIEURE}

Comme le vagin, le vestibule vaginal est aplati dorso-ventralement à son extrémité crâniale. Cette disposition transversale se transforme progressivement en un aplatissement latéro-latéral qui l'oriente caudalement dans le même sens que l'orifice vulvaire.

Chez la vache adulte, le vestibule vaginal mesure en moyenne 10 à $12 \mathrm{~cm}$ de longueur. En raison de la position de l'orifice vulvaire, son plafond est un peu plus court que son plancher. Ce dernier est en outre oblique dorso-crânialement.

Chez la vache gestante, le vestibule vaginal est entraîné vers l'avant par l'utérus gravide. Sa longueur augmente progressivement. Au moment du part, son diamètre est considérablement accru.

\section{I.B.2.) CONFORMATION INTERIEURE}

La CAVITE VESTIBULAIRE est virtuelle à l'état de repos. Elle est tapissée par une muqueuse lisse, souvent rosée, mais aussi plus ou moins pigmentée selon les races. Cette muqueuse est recouverte d'un mucus épais pendant la plus grande partie du cycle. Au moment de l'cestrus, elle se congestionne et le mucus devient plus abondant et plus fluide.

La cavité vestibulaire communique crânialement avec la cavité vaginale par l'ORIFICE VAGINAL (Ostium vaginae) dont l'emplacement est marqué par l'hymen.

L'HYMEN (Hymen) de la femelle zébu est réduit à une ébauche de cloison membraneuse qui résulte de l'adossement des muqueuses vaginale et vestibulaire. Sa hauteur dépasse rarement 5 à $6 \mathrm{~mm}$. Il n'est souvent développé que sur les portions ventrale et latérales du conduit génital dont le plafond reste alors parfaitement lisse. 


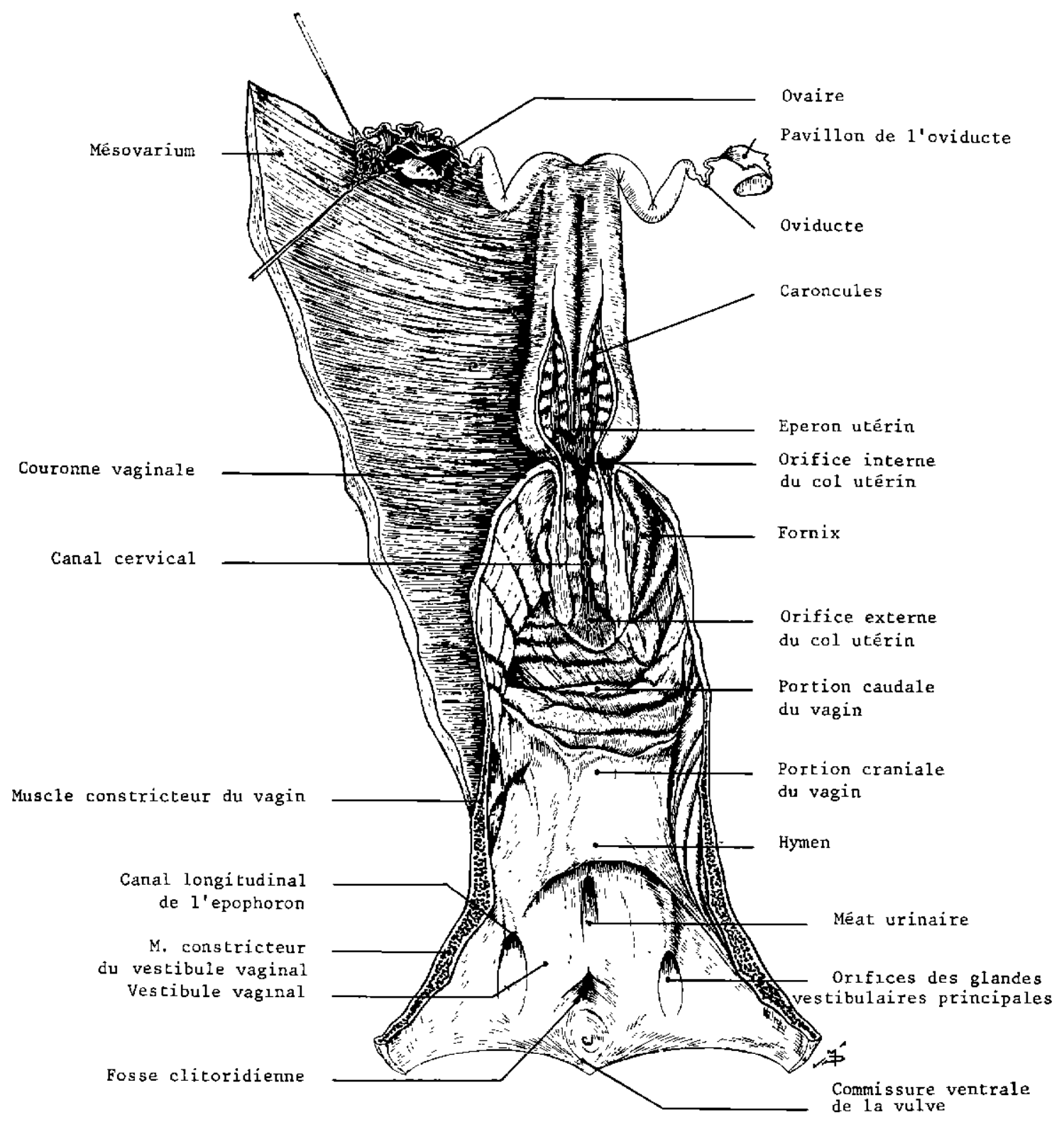

Conformation intérieure du tractus génital de la femelle zêbu

(vue dorsale après ouverture du plafond des portions gestative et copulatrice)

L'ORIFICE EXTERNE DE L'URETHRE ou MEAT URINAIRE (Ostium urethrae externum) est situé sur le plancher du vestibule vaginal, dans le plan médian et juste en arrière de l'hymen. Il est de forme ovalaire et orienté crânio-caudalement. Son bord dorso-crânial affecte la disposition d'une valvule transversale à bord libre concave dont la fonction est de rabattre l'urine vers la commissure ventrale de la vulve au moment de la miction.

A proximité du méat urinaire, le plancher du canal uréthral forme un diverticule ventral appelé le DIVERTICULE SOUS-URETHRAL (Diverticulum suburethrale).

A deux, trois centimètres de l'orifice uréthral externe, et caudo-latéralement par rapport à lui, se trouvent les orifices des CANAUX LONGITUDINAUX DE L'EPOOPHORON (Ductus epoophori longitudinalis dexter et sinister) qui furent longtemps décrits sous le nom de canaux 
de Gartner. Chacun de ces orifices est placé au fond d'une dépression ovalaire crânio-caudale et se prolonge oralement par un canal à extrémité aveugle dans le chorion de la muqueuse. Parfois très courts, les canaux longitudinaux de l'époophoron ne mesurent que quelques millimètres. Le plus souvent, ils se prolongent jusqu'au vagin dont ils peuvent atteindre l'extrémité crâniale.

Caudalement, à proximité de la vulve, les parois latérales du vestibule vaginal présentent deux dépressions ovalaires à grand axe crânio-caudal, au fond desquelles débouchent par de très fins orifices les canaux excréteurs des glandes vestibulaires principales. Par analogie avec le tractus génital mâle, ces formations méritent le nom de SINUS VESTIBULAIRE (Sinus vestibularis).

\section{I.B.3.) STRUCTURE}

La paroi du vestibule vaginal comprend trois tuniques, une adventice, une musculeuse et une muqueuse.

LA TUNIQUE ADVENTICE (Tunica adventitia) du vestibule vaginal a la même structure que celle de la portion rétro-péritonéale du vagin. Elle est formée de tissu conjonctif riche en fibres élastiques et en lobules adipeux. Dans la profondeur, elle prend une disposition concentrique autour de la tunique musculaire. A la périphérie, elle se confond avec le tissu conjonctif de l'espace rétro-péritonéal.

LA TUNIQUE MUSCULEUSE (Tunica muscularis) est composée d'une couche profonde de fibres musculaires lisses disposées longitudinalement et d'une couche superficielle de fibres musculaires striées à orientation circulaire qui constituent le muscle constricteur du vestibule.

LE MUSCLE CONSTRICTEUR DU VESTIBULE VAGINAL ( $M$. constrictor vestibuli) prolonge dorsalement le muscle uréthral de la femelle. Il enveloppe ventralement et latéralement le vestibule vaginal et se prolonge dorsalement par des faisceaux aponévrotiques qui se terminent sur le bord supérieur du vestibule et les faces latérales du rectum. La contraction de ce muscle provoque la diminution du diamètre de la cavité vestibulaire pendant le coït. Par ses faisceaux ventraux le muscle constricteur du vestibule provoque en outre la fermeture de l'orifice uréthral au moment du rapprochement sexuel.

LA TUNIQUE MUQUEUSE (Tunica mucosa) est composée d'un épithélium et d'un chorion.

LE CHORION DE LA MUQUEUSE (Lamina propria mucosae) est formé de tissu conjonctif lâche, riche en fibres élastiques, en formations Iymphoïdes, en glandes et en tissu érectile.

Les glandes vestibulaires du chorion se subdivisent en glandes vestibulaires accessoires, diffuses et en glandes vestibulaires principales, conglomérées.

LES GLANDES VESTIBULAIRES ACCESSOIRES (Gl. vestibularis minores) sont abondantes au niveau du plancher vaginal, et plus particulièrement en région crâniale où elles se condensent de part et d'autre du méat uréthral.

LES GLANDES VESTIBULAIRES PRINCIPALES ( $G$. vestibularis major dexter et sinister), conglomérées mais polystomatiques, sont situées dans le chorion de la portion caudale des parois latérales du vestibule. Leurs canaux débouchent, par de multiples orifices, dans les sinus vestibulaires. Qu'elles soient accessoires ou principales, les formations glandulaires du vestibule vaginal assurent, avec le mucus cervico-vaginal, la lubrification des voies génitales de la femelle au moment du coït.

LE BULBE VESTIBULAIRE (Bulbus vestibuli) est une formation érectile, homologue du bulbe uréthral ou bulbe du pénis du mâle. Moins individualisé que chez les taurins, il se présente sous l'aspect de deux formations diffuses localisées dans les parois latérales du vestibule, sous le muscle constricteur. Il est formé d'espaces caverneux situés dans la portion correspon- 
dante du chorion de la muqueuse. Il assure de concert avec le muscle constricteur du vestibule, la coaptation des organes copulateurs du mâle et de la femelle au moment du coït.

L'EPITHELIUM DE LA TUNIQUE MUQUEUSE (Lamina epithelialis mucosae) est de type stratifié pavimenteux et non corné, comme celui de la portion caudale et lisse du vagin. Comme ce dernier, il subit des transformations structurales périodiques au cours du cycle cestral.

\section{I.C.) La vulve}

La VULVE (Vulva; Pudendum femininum) correspond à la portion externe des organes génitaux de la femelle.

\section{I.C.1.) CONFORMATION EXTERIEURE}

Extérieurement, l'orifice vulvaire se présente sous la forme d'une fente étroite orientée dorso-ventralement. Elle est limitée latéralement par deux lèvres verticales qui s'unissent dorsalement et ventralement au niveau de deux commissures.

L'ORIFICE VULVAIRE ou fente vulvaire (Rima pudendi) est situé au-dessous de la région anale dont il est séparé par le périné. Il mesure 8 à $10 \mathrm{~cm}$ de longueur.

Les LEVRES DE LA VULVE (Labiae pudendi) sont peu épaisses. Elles s'amincissent encore dorsalement au niveau de la commissure correspondante. Leur face externe est recouverte d'une peau fine, pigmentée ou non selon les sujets et peu riche en poils.

La COMMISSURE DORSALE DE LA VULVE (Commissura labiorum dorsalis) est étroite et située dans le plan même du périné à 4 ou $5 \mathrm{~cm}$ de l'anus.

La COMMISSURE VENTRALE DE LA VULVE (Commissura labiorum ventralis) est saillante et aiguë. Située au-dessous du niveau de l'arcade ischiatique, elle est en relief par rapport aux téguments avoisinants, de telle sorte que sur l'animal en position quadrupédale, l'orifice vulvaire est dans l'ensemble vertical alors que le plan cutané avoisinant est oblique ventrocrânialement.

\section{I.C.2.) CONFORMATION INTERIEURE}

Le revers interne des lèvres vulvaires est tapissé d'une muqueuse de même type que la muqueuse vestibulaire et peut, comme cette dernière, être pigmenté ou non selon les races. Le raccordement du revêtement muqueux interne avec le revêtement cutané externe s'effectue sans transition au niveau du bord libre.

Le revers interne de la commissure ventrale de la vulve loge l'extrémité libre du clitoris dans une dépression du plancher vulvaire, la FOSSE CLITORIDIENNE (Fossa clitoridis). Il est tapissé par la muqueuse vulvaire qui le recouvre d'un repli transversal, le CAPUCHON ou PREPUCE DU CLITORIS (Preputium clitoridis).

\section{I.C.3.) LE CLITORIS}

Le CLITORIS (Clitoris) est un organe érectile, homologue de la verge du mâle. Il est formé comme cette dernière par un corps caverneux et un corps spongieux du gland rudimentaire mais il est dépourvu d'urèthre et ne présente qu'un seul muscle annexe : le muscle ischiocaverneux.

Le CORPS DU CLITORIS (Corpus clitoridis) est légèrement aplati d'un côté à l'autre. Il mesure 4 à $5 \mathrm{~cm}$ de long pour un diamètre de 10 à $12 \mathrm{~mm}$. Il s'insère sur l'arcade ischiatique par deux RACINES (Crura clitoridis) recouvertes par deux MUSCLES ISCHIO. CAVERNEUX ( $M$. ischio cavernosus) grêles et courts. L'extrémité libre du clitoris est orientée ventro-caudalement. Elle est recouverte par un corps spongieux du gland, légèrement renflé mais rudimentaire qui constitue le GLAND DU CLITORIS (Glans clitoridis). 


\section{I.C.4.) STRUCTURE DE LA VULVE}

La FACE EXTERNE DES LEVRES DE LA VULVE est tapissée d'un revêtement cutané peu épais, mais très riche en glandes sébacées et sudoripares. La FACE INTERNE et la ZONE CLITORIDIENNE sont recouvertes d'un épithélium stratifié pavimenteux (Epithelium stratificatum) dépourvu de glandes et de poils. La lame conjonctive qui s'interpose entre les deux revêtements épithéliaux représente un hypoderme épais, riche en vaisseaux, en fibres musculaires lisses et en terminaisons nerveuses.

Le MUSCLE CONSTRICTEUR DE LA VULVE ( $M$. constrictor vulvae) est formé de fibres musculaires striées. Il se subdivise en deux portions latérales qui s'unissent au niveau des commissures. Dorsalement, les fibres du muscle constricteur de la vulve sont en continuité avec celles du muscle sphincter externe de l'anus. Ventralement, elles s'épanouissent en faisceaux qui se dirigent vers le clitoris et la face profonde du tégument de la région périnéale. Ce muscle est donc à la fois constricteur de la vulve, releveur du clitoris et tenseur du périné.

Le MUSCLE RETRACTEUR DU CLITORIS ( $M$. retractor clitoridis) ou " Muscle suspenseur de la vulve »est formé de deux lames conjonctivo-musculaires très pâles qui se fixent dorsalement sur la face ventrale de la dernière vertèbre sacrée et des premières vertèbres coccygiennes, croisent latéralement le rectum, atteignent le vestibule vaginal, s'engagent sous le muscle constricteur du vestibule et se terminent par des faisceaux qui s'irradient dans ce muscle, dans le muscle constricteur de la vulve, dans les lèvres vulvaires et sur le clitoris.

Le CORPS CAVERNEUX DU CLITORIS (Corpus cavernosum clitoridis) comme le corps caverneux de la verge, est formé par un tissu érectile, enveloppé d'une lame périphérique fibroélastique (Tunica albuginea corpus cavernosum) et divisé médialement, par une cloison fibreuse (Septum corporum cavernosum), particulièrement développée dans la partie libre, en deux masses symétriques.

Le GLAND DU CLITORIS est constitué de tissu conjonctif riche en vaisseaux sanguins mais dépourvu d'aréoles comparables à celles du corps spongieux du gland du mâle.

\section{MOYENS DE FIXITE DE LA PORTION COPULATRICE}

La portion copulatrice du tractus génital de la femelle est fixée caudalement par sa continuité avec la région périnéale. Crânialement elle est unie à l'utérus par l'intermédiaire du complexe col fornix. Le péritoine pelvien et le conjonctif rétro-péritonéal contribuent en outre à maintenir l'organe en place.

\section{II.A.) Les culs-de-sac pelviens du péritoine}

Le péritoine ne se prolonge pas jusqu'au fond de la cavité pelvienne. Avant d'atteindre la région périnéale, il se réfléchit des parois aux viscères en formant des culs-de-sac.

\section{II.A.1.) LE CUL-DE-SAC RECTO-GENITAL}

Le CUL-DE-SAC RECTO-GENITAL (Excavatio recto-genitalis) est très profond. Il se prolonge très loin vers l'arrière en se rétrécissant graduellement. Il se termine en doigt de gant à quelques millimètres seulement du périné. L'étendue de ce cul-de-sac et le volume important du fornix chez la femelle zébu devraient permettre dans cette espèce une application facile de la castration par voie vaginale.

\section{II.A.2.) LE CUL-DE-SAC VESICO-GENITAL}

Le CUL-DE-SAC VESICO-GENITAL (Excavatio vesicogenitalis) est court. Il ne dépasse pas caudalement le niveau du col de la vessie et n'interfère par conséquent sur le vagin que dans la zone fornicale. 
Entre les deux culs-de-sac, recto-génital et vésico-génital, le péritoine se réfléchit sur les parois latérales du vagin selon une ligne oblique caudalement et dorsalement, si bien que le bord dorsal, la moitié crânio-dorsale des faces latérales et la partie la plus crâniale du bord ventral de l'organe sont seuls recouverts par le péritoine. Encore faut-il, à propos de cette limite, tenir compte du fait que la laxité du conjonctif sous-séreux permet une certaine mobilité de la lame mésothéliale par rapport à la surface de l'organe lorsque le péritoine est entraîné plus ou moins loin, crânialement, par la vessie ou le rectum en état de réplétion.

\section{II.B.) L'espace rétro-péritonéal}

L'ESPACE RETRO-PERITONEAL (Spatium retroperitoneale), compris entre les culs-desac péritonéaux et le périné est occupé par un tissu conjonctif lâche et abondant. Les fibres conjonctivo-élastiques qui le constituent unissent la portion correspondante du tractus génital aux parois de la cavité pelvienne, au rectum et à la vessie.

\section{IRRIGATION ET INNERVATION}

\section{III.A.) Artères de la portion copulatrice du tractus génital de la femelle zébu}

La portion copulatrice reçoit son irrigation de l'artère honteuse inteme et de l'artère vaginale.

\section{III.A.1.) ARTERE HONTEUSE INTERNE}

L'ARTERE HONTEUSE INTERNE ( $A$. pudenda interna) est une branche terminale de l'artère iliaque interne ( $A$. iliaca interna). Elle naît au niveau de la petite échancrure sciatique, se dirige obliquement caudo-ventralement dans le conjonctif rétro-péritonéal, croise les faces latérales du vagin, atteint le vestibule vulvaire, s'engage sous les muscles constricteur du vestibule et constricteur de la vulve et se termine par deux branches : l'artère périnéale ventrale et l'artère clitoridienne.

Elle émet dans son trajet plusieurs branches collatérales.

L'ARTERE VAGINALE ( $\boldsymbol{A}$. vaginalis) peut être la première branche collatérale de l'artère honteuse interne. Elle naît le plus souvent sur l'artère iliaque interne et sera étudiée plus loin.

L'ARTERE URETHRALE ( A. urethralis) peut naître sur l'artère honteuse interne ou sur l'artère vaginale. Elle croise les faces latếrales du vestibule et se distribue par un trajet récurrent à l'urèthre féminin.

L'ARTERE VESTIBULAIRE ( $A$, vestibularis), simple ou multiple, longe les faces latérales du vestibule vaginal dans lequel elle se distribue.

L'ARTERE PERINEALE DORSALE ( $A$. perinealis dorsalis) naît du bord dorso-caudal de l'artère honteuse interne. Elle longe le bord supérieur du vestibule puis atteint le périné qu'elle irrigue de la commissure dorsale de la vulve à l'anus. Dans son trajet, elle émet une ARTERE RECTALE CAUDALE ( $A$. rectalis caudalis) qui irrigue la portion terminale du rectum et des RAMEAUX LABIAUX (Rami labiales) pour la commissure dorsale de la vulve et les portions avoisinantes des lèvres.

L'ARTERE PERINEALE VENTRALE (A. perinealis ventralis) du zébu est très grêle. Elle est habituellement une branche terminale de l'artère honteuse interne, mais peut aussi être une collatérale de l'artère périnéale dorsale. Elle longe les lèvres vulvaires en émettant des RAMEAUX LABIAUX (Rami labiales) et se prolonge, en dessous de la vulve, jusqu'à la mamelle dans laquelle elle se termine par de très fins RAMEAUX MAMMAIRES (Rami mammarii). 
N. honteux interne

A. honteuse interne

A. recta1e moyenne

A. périnéale dorsale

Plexus hypogastrique

A. vaginale

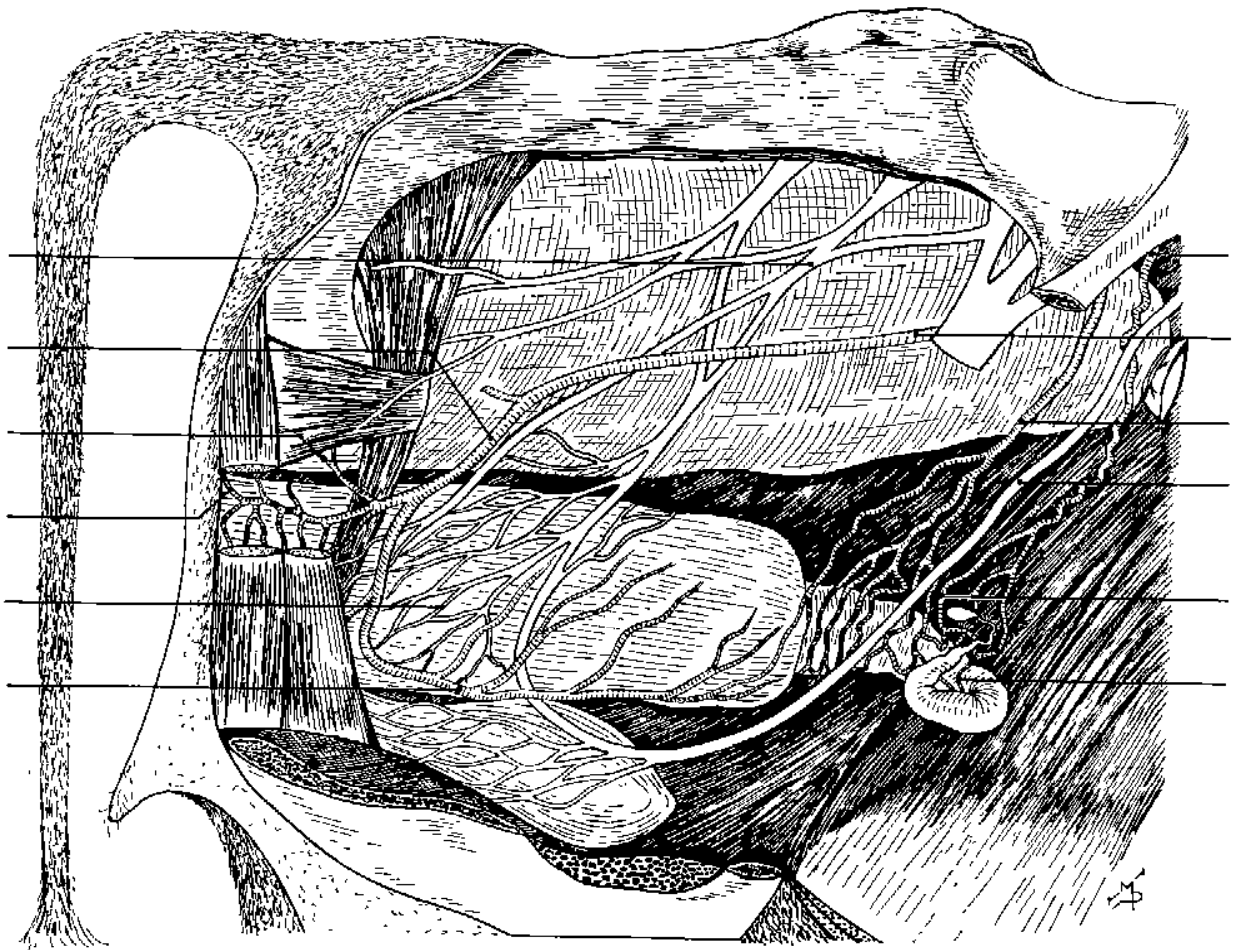

Les artères et les nerfs du Lractus génital de 1a femelle zébu

(vue latẹrale droite, organcs en plize après ouverture du bassin)

,
A, ovarienne

A. 11iaque interne

A. uLérine

N. hypogastrique

R. tubaire cranial de

1'A. ovarienne

R. utërin de I'A. ovarienne

havarienne 
L'ARTERE CLITORIDIENNE ( $A$. clitoridis) homologue de l'artère penienne du mâle aborde le clitoris par sa racine et se termine dans le corps caverneux en formant l'ARTERE PROFONDE DU CLITORIS ( $A$ profunda clitoridis). Elle émet dans son trajet une ARTERE DU BULBE VESTIBULAIRE ( $A$. bulbi vestibuli) et une ARTERE DORSALE DU CLITORIS (A. dorsalis clitoridis) homologues des artères bulbaire et dorsale du pénis du mâle.

\section{III.A.2.) L'ARTERE VAGINALE}

L'ARTERE VAGINALE ( $A$. vaginalis) naît plus souvent sur la portion terminale de l'artère iliaque interne que sur l'artère honteuse interne. Elle se caractérise chez le zébu par l'absence de rameaux utérins.

Dès son origine, elle se dirige ventro-caudalement pour atteindre l'extrémité dorsale des faces latérales de la région vestibulo-vaginale. Elle décrit alors une courbe à concavité crâniale qui l'amène au bord ventral du vagin qu'elle longe ensuite pour se terminer en s'anastomosant à plein canal avec le rameau crânial de l'artère utérine.

Dans son trajet, l'artère vaginale émet par son bord dorsal une ARTERE RECTALE MOYENNE ( $A$. rectalis media), des RAMEAUX VAGINAUX (Rami vaginalis). Par son bord ventral, elle donne naissance à une ARTERE VESICALE ( $A$. vesicalis) qui se distribue à la totalité de la vessie et résume à elle seule les artères vésicales crâniale et caudale de $B o s$ taurus.

\section{III.B.) Veines de la portion copulatrice}

La portion copulatrice du tractus génital de la femelle zébu est drainée par les veines honteuse interne et vaginale.

\section{II.B.1.) LA VEINE HONTEUSE INTERNE}

La VEINE HONTEUSE INTERNE ( $V$, pudenda interna) naît dans la région vestibulaire par la confluence de la veine vestibulaire et de la veine clitoridienne. D'abord située ventralement, sous les muscles constricteurs de la vulve et du vestibule, elle croise ensuite les parois latérales de la section copulatrice et s'unit au niveau de la petite échancrure sciatique à la veine fessière caudale avec laquelle elle forme la veine iliaque interne.

La VEINE CLITORIDIENNE ( $V$. clitoridis) se constitue de façon variable par la confluence de la VEINE PROFONDE DU CLITORIS ( $V$. profunda clitoridis) de la VEINE DORSALE DU CLITORIS ( $V$. dorsalis clitoridis) et de la VEINE DU BULBE VESTIBULAIRE ( $V$. bulbi vestibuli).

La VEINE VESTIBULAIRE ( $V$. vestibularis) forme avec le rameau vestibulaire de la veine vaginale une arcade veineuse qui longe le bord ventral des faces latérales de la portion caudale du vagin et du vestibule. Cette ARCADE VEINEUSE VAGINO-VESTIBULAIRE reçoit dorsalement des affluents génitaux et ventralement des affluents uréthraux.

Sur son trajet, la veine honteuse interne reçoit de manière variable une VEINE PERINEALE VENTRALE ( $V$. perinealis ventralis), des RAMEAUX LABIAUX (Rami labialis), une VEINE PERINEALE DORSALE ( $V$. perinealis dorsalis) des RAMEAUX RECTAUX (Rami rectalis) des RAMEAUX VAGINAUX et des RAMEAUX VESTIBULAIRES (Rami vaginalis et vestibularis). Elle reçoit aussi un volumineux affluent: la veine vaginale.

\section{II.B.2.) LA VEINE VAGINALE}

La VEINE VAGINALE ( $V$. vaginalis) prolonge caudalement la veine marginale de l'utérus dont la portion sinueuse, déjà décrite, draine la moitié rostrale du vagin. Très volumineuse, la veine vaginale croise obliquement la paroi latérale du vagin en formant une courbe à concavité crâniale. Elle se termine dans l'artère honteuse interne. 
A son origine, elle reçoit ventralement la VEINE VESICALE ( $V$. vesicalis). Le RAMEAU VESTIBULAIRE (qui correspond à la racine rostrale de l'arcade veineuse vestibulo-vaginale) et des RAMEAUX VAGINAUX, à disposition variable, sont aussi des affluents de la veine vaginale.

\section{III.C.) Lymphatiques de la portion copulatrice}

Les vaisseaux lymphatiques sont abondants au niveau du vagin. Ils y forment des plexus dans le chorion de la muqueuse, la tunique musculaire et l'adventice. Dans le vestibule et la vulve, les plexus vasculaires sont plus discrets que dans le vagin. Les vaisseaux efférents du vagin rejoignent les GANGLIONS ILIAQUES INTERNES (Lymphodoni iliaci mediales) et LOMBO-AORTIQUES (Lymphonodi lumboles aortici). Ceux du vestibule et de la vulve sont drainés par les GANGLIONS MAMMAIRES (Lymphonodi mammarii).

\section{III.D.) Les nerfs de la portion copulatrice}

La portion copulatrice du tractus génital de la femelle est innervée par le nerf honteux interne et des rameaux issus du plexus hypogastrique.

\section{III.D.1.) LE NERF HONTEUX INTERNE}

Le NERF HONTEUX INTERNE ( $N$. pudendus) se distribue aux muscles annexes et à la muqueuse par des arborisations libres (nombreuses dans la région vaginale) ou des terminaisons corpusculaires spécialisées (plus particulièrement dans les régions vulvo-vestibulaire et clitoridienne).

\section{III.D.2.) LE PLEXUS HYPOGASTRIQUE}

Le PLEXUS hyPOGASTRIQUE (Plexus pelvinus) forme à la périphérie de l'organe des plexus préviscéraux desquels naissent des rameaux nerveux qui se distribuent dans la paroi et plus particulièrement dans les formations érectiles.

\section{CONCLUSION}

En raison des nombreuses analogies morphologiques et structurales qui rapprochent les deux espèces domestiques du genre Bos, les organes génitaux de la femelle zébu ont longtemps été considérés comme identiques à ceux des taurins des régions tempérées et les importantes différences fonctionnelles qui les caractérisent, systématiquement rapportées aux difficiles conditions d'environnement qu'elle subit. L'étude anatomique montre qu'en réalité d'importantes différences morphologiques et structurales caractérisent chacune des deux espèces.

Les principales particularités anatomiques des organes génitaux de la femelle zébu peuvent être répertoriées sous trois rubriques respectivement consacrées à leur morphologie, à leur topographie et à leur structure.

\section{MORPHOLOGIQUEMENT}

L'OVAIRE du zébu est plus petit et moins lourd que celui des taurins. Sa forme est aussi moins régulière. Tantôt en amande aplatie d'un côté à l'autre, comme celui de Bos taurus, il est aussi souvent sub-ovoïde, à bords dorsal et ventral presque parallèles et à pôles subégaux.

L'ovaire de Bos indicus est situé dans une bourse ovarique profonde et ouverte en dedans en raison de la longueur et de la disposition particulière du mésosalpinx dont le bord libre se replie dorso-médialement.

La PORTION GESTATIVE du tractus génital de la femelle zébu est caractérisée par un corps utérin virtuel qui classe l'utérus de cette espèce dans la catégorie Uterus bipartitus. 
Le col utérin du zébu interfère largement sur le vagin. Dans de nombreux cas il est même entièrement intra-vaginal. Le canal cervical est rectiligne ou très peu sinueux; ses parois sont garnies de papilles très basses et son orifice externe est infundibuliforme. Il peut en conséquence être beaucoup plus facilement cathétérisé que celui des taurins.

LA PORTION COPULATRICE du tractus génital de la femelle zébu possède un fornix profond qui se traduit extérieurement par un fort relief en couronne, la couronne vaginale, d'où émergent crânialement les deux comes utérines placées côte à côte. La muqueuse vaginale se subdivise en deux portions: une portion crâniale d'aspect plissé en raison de la présence de bourrelets circulaires transversaux et une portion caudale qui est lisse. L'hymen qui sépare le vagin du vestibule vaginal est toujours rudimentaire. Les glandes vestibulaires principales sont de type polystomatique.

LES ARTERES du tractus génital de la femelle zébu se caractérisent par une distribution strictement glandulaire et tubaire de l'artère ovarienne et par le fait que l'artère vaginale limite son territoire d'irrigation à la portion copulatrice. Aucun de ses rameaux n'atteint le col ou le corps utérins.

LA VEINE MARGINALE DE L'UTERUS est simple chez la génisse mais se complique chez la vache multipare. Elle se différencie caudo-crânialement en une portion sinueuse qui draine le vagin, une portion plexiforme qui reçoit ses affluents de la portion caudale des cornes utérines, une portion en anse qui draine l'anse cornuale et une portion rectiligne qui rejoint la veine ovarienne .Elle reçoit la veine tubo-utérine qui draine une partie de l'oviducte et la portion crâniale des cornes.

\section{TOPOGRAPHIQUEMENT}

Le tractus génital de la femelle zébu a une disposition spatiale qui résulte des rapports qu'il entretient avec son appareil suspenseur. Le ligament large s'insère en effet de façon très particulière sur les cornes utérines. Dans leur tiers caudal ou rectiligne, les cornes sont disposées horizontalement, côte à côte; le ligament large s'insère sur leur bord ventral. Le tiers moyen des comes est disposé en anse à convexité orientée latéro-ventralement; il est suspendu au ligament large qui s'insère dans sa concavité. Le tiers crânial ou portion tubaire des cornes utérines prolonge crânio-dorsalement et médialement la portion en anse; le ligament large s'insère sur son bord dorsal. Au total, la partie la plus mobile de l'utérus, qui correspond aux deux tiers crâniaux des cornes, est suspendue à l'extrémité libre du méso. Ce mode de fixation diffère considérablement de celui qu'on observe chez Bos taurus. Dans ce dernier, en effet, les cornes utérines, sur toute leur longueur, reçoivent ventralement l'insertion du mésométrium. Cette particularité anatomique explique que chez Bos indicus les torsions utérines sont rares ou inexistantes alors qu'elles sont relativement fréquentes chez Bos taurus.

Le cul-de-sac péritonéal recto-génital du zébu est très profond. Il s'étend caudalement le long du bord dorsal du vagin jusqu'au voisinage du périné. Cette topographie particulière ainsi que l'ampleur du fornix, déjà signalée, doivent en principe rendre facile, dans cette espèce, la pratique de l'ovariectomie par voie vaginale.

Au début de la gestation, l'œuf se fixe habituellement dans la portion moyenne de la corne gravide. Lors du développement fœtal, c'est donc l'anse cornuale qui subit l'accroissement de volume le plus précoce et le plus important. En grossissant, elle migre ventro-crânialement et s'engage, le plus souvent, dans la loge sus-omentale. Le reste du tractus génital subit passivement les conséquences de cette migration. Entraîné par le poids de la corne gravide, le vagin s'allonge et le col utérin se rapproche du bord crânial du pubis qu'il atteint vers le $6^{\mathrm{e}}$ mois de la gestation. Pour la même raison, l'ovaire et l'oviducte sont entraînés ventralement et crânialement dans une migration que limite le mésovarium proximal et le ligament suspenseur de l'ovaire.

Après la mise bas, l'utérus involue et le ligament large se rétracte. Ni l'un ni l'autre cependant, ne reprennent leur disposition primitive. Après chaque gestation, le tractus génital et son 
méso de suspension ont des dimensions supérieures à celles qu'ils avaient dans la période d'intervêlage précédente. Chez la vache multipare, le vagin est ainsi d'autant plus long et le col se trouve en position d'autant plus crâniale que le sujet a donné naissance à un plus grand nombre de veaux. De même, les cornes utérines sont d'autant plus volumineuses et en position d'autant plus abdominale et plus basse que les gestations ont été plus fréquentes. La portion moyenne des cornes dans laquelle se fixe primitivement l'œuf subit aussi d'importantes variations morphologiques. En V à pointe ventro-latérale et à branches courtes chez la génisse, elle s'allonge, grossit peu à peu et prend une forme en $U$ dont la portion intermédiaire est d'autant plus ventrale et plus longue que l'animal a eu une vie génitale plus active.

Les variations topographiques post-gravidiques de l'ovaire résultent de l'allongement du mésovarium proximal et du déplacement progressif de l'utérus vers les parties déclives de la région abdominale caudale. Les premières gestations provoquent une migration crânio-ventrale de la gonade dont la limite rostrale correspond au niveau de l'insertion du bord oral du ligament large sur la région sous-lombaire. Cette limite crâniale atteinte, les gestations suivantes se traduisent par un déplacement ventral de la glande qui résulte de l'augmentation progressive de la hauteur du mésovarium proximal, et par l'inversion de son orientation qui est la conséquence du repliement de la portion rostrale de l'appareil suspenseur. Ce dernier dont les gestations successives augmentent la laxité est peu à peu entraîné médio-caudalement par la portion tubaire des cornes utérines. La face latérale de l'ovaire devient médiale, son pôle crânial devient caudal et l'ouverture de la bourse ovarique s'applique sur la face interne du mésométrium.

\section{STRUCTURALEMENT}

Les différences les plus importantes siègent au niveau de l'ovaire dont le fonctionnement conditionne l'évolution cyclique du reste du tractus génital.

L'ovaire de la femelle zébu est caractérisé par la présence d'une zone de recouvrement péritonéal qui s'étend sur une portion plus ou moins grande de sa face médiale, peut la recouvrir toute et parfois même déborder sur son pôle utérin et son bord ventral. Dans cette zone, une lame conjonctive sous-séreuse double la lame séreuse épithéliale. Elle empêche les follicules en croissance qui migrent dans sa direction d'atteindre le stade de la déhiscence. Le champ d'ovulation de la femelle zébu est donc d'autant moins étendu que la zone de recouvrement péritonéal est plus importante.

Cette particularité explique les chaleurs anovulatoires et les chaleurs de durée anormalement longue qui ont été signalées dans cette espèce.

Les follicules évolutifs de Bos indicus ont la même structure que ceux de Bos taurus. Chez le premier, cependant, il est intéressant de noter qu'au cours du pro-œstrus, plusieurs follicules cavitaires entament de façon régulière le processus de la maturation et qu'un seul arrive habituellement au stade de la déhiscence. Les autres involuent plus ou moins précocement. Cette observation est en accord avec la rareté relative de la gemelléité chez le zébu $(0,36$ p. 100 selon nos observations).

L'ovulation est tardive chez Bos indicus. Elle se produit le plus souvent vers la $14^{\mathrm{e}}$ heure qui suit la fin des manifestations extérieures de l'œstrus. Cette particularité justifie l'insémination artificielle en fin de chaleur. Elle pourra être prise en considération lorsque l'insémination artificielle se pratiquera de façon courante dans les régions tropicales.

La femelle zébu présente aussi quelques particularités d'involution folliculaire. Au type classiquement décrit des follicules atrétiques à dégénérescence épithéliale rapide et complète s'ajoutent des formes dans lesquelles la Granulosa ou l'ovocyte persistent plus ou moins longtemps. Les follicules kystiques vrais du zébu sont très rares et peut-être inexistants. Le plus souvent, cette forme d'involution se traduit par une brève phase de prolifération et de sécrétion des cellules folliculeuses bientôt suivie par une prolifération des thèques et du stroma ovarien qui démembrent le kyste en voie de formation. Les grands follicules cavitaires et les follicules mûrs qui n'atteignent pas le stade de la déhiscence subissent une involution particulière et rapide qui est 
caractérisée par la transformation de la thèque en une série de lamelles concentriques pauvres en cellules et riches en fibres collagènes. Nous proposons, pour désigner cette forme d'involution qui n'a pas encore été décrite, le terme de «follicule lamelleux».

Les corps progestatifs du zébu diffèrent de ceux des taurins par leur involution tardive et la longue durée de leur période de dégénérescence. Alors que chez Bos taurus le corps jaune présente toujours de nets signes de dégénérescence au pro-œstrus et à l'œstrus du cycle suivant, celui de Bos indicus, à la même période, n'est qu'au début de son stade d'involution. Cette particularité rend difficile, dans cette espèce, la diagnose histologique du di-œstrus.

Les corps gestatifs du zébu ont une évolution variable. Sur un certain nombre de sujets leur activité se prolonge, comme chez les taurins, pendant toute la durée de la gestation. Souvent aussi, ils régressent et peuvent alors être remplacés ou supplémentés. Le remplacement est caractérisé par le développement d'un nouveau corps gestatif identique au premier. Il peut s'effectuer une, deux et peut-être même trois fois au cours de la gestation. Les périodes les plus favorables à ce remplacement sont les $4^{\mathrm{e}}$ et $7^{\mathrm{e}}$ mois au cours desquels des cstrus vrais ont été signalés par certains auteurs. La supplémentation est caractérisée par la transformation d'un ou plusieurs follicules cavitaires moyens ou grands en glande endocrine. Dans cette modalité, la transformation des cellules folliculeuses en cellules progestatives et celle des cellules thécales en cellules œstrogènes s'effectue in situ. Elle aboutit à la constitution d'un îlot central de granulolutéocytes qu'entoure une couronne de lutéocytes thécaux. Les corps gestatifs du zébu peuvent enfin disparaître complètement sans être remplacés ni supplémentés. Nous avons observé ce phénomène deux fois sur des sujets ayant dépassé le $6^{e}$ mois de la gestation. Il est vraisemblable que, comme dans d'autres espèces, le relais hormonal soit pris alors par le placenta.

Nous avons observé enfin, chez la femelle zébu, des périodes d'anœstrus au cours desquelles aucun organite susceptible d'être rattaché à un cycle en cours ne peut être identifié. Ces périodes varient de la durée d'un cycle à trois mois ou plus. En zone sahélienne du Sénégal, on peut les observer pendant toute l'annéc mais elles sont plus fréquentes en saison sèche qu'en saison des pluies. Fonctionnellement, elles se présentent sous deux aspects. Dans le premier, l'ovaire est complètement au repos et ne contient plus que des follicules primaires, des follicules cavitaires petits et moyens et des corps progestatifs en état de dégénérescence plus ou moins avancée selon que l'anœstrus dure depuis un temps plus ou moins long. Le repos sexuel peut alors être rapporté à une insuffisance des sécrétions gonadotropes de l'hypophyse ou à une inhibition temporaire du récepteur ovarien. Le deuxième aspect que peut présenter l'ovaire du zébu en repos sexuel est caractérisé par l'existence de poussées évolutives périodiques qui toutes avortent systématiquement. Des follicules cavitaires petits et moyens commencent l'évolution mais n'atteignent jamais le stade de la maturation. Ils involuent sous la forme de follicules lamelleux. Par analogie structurale avec la maladie polykystique des ovaires de la femme, cette forme d'ancstrus peut être fonctionnellement expliquée par une activité gonadotrope F.S.H. normale qui explique les poussées de croissance folliculaire, et une activité gonadotrope L.H. insuffisante ou nulle qui serait responsable de l'absence d'ovulation.

D'importantes différences macroscopiques et microscopiques caractérisent donc le tractus génital du zébu. Parmi ces caractères différentiels, certains sont spécifiques comme la disposition des cornes utérines ou la zone de recouvrement péritonéal de l'ovaire. D'autres, comme les images d'ancstrus que nous avons observées, nécessitent des travaux complémentaires pour que soit déterminée la mesure dans laquelle ils correspondent à des caractères spécifiques ou à des conséquences fonctionnelles de l'action du biotope.

\section{SUMMARY}

\section{The Zebu cow sexual organs}

The Zebu cow sexual organs are described. Anatomical differences existing with regard to Bos taurus are emphasized. 


\section{RESUMEN}

\section{Los organos genitales de la hembra cebú}

Se describen en detalle los órganos genitales de la hembra cebú y se pone en evidencia las diferencias anatómicas existiendo con relación a Bos taurus.

\section{BIBLIOGRAPHIE}

AGBA (K. C.). Particularités anatomiques et fonctionnelles des organes génjtaux de la femelle zébu. Thèse Duct. vét. Dakar, 1975, no 12.

CUQ (P.). Bases anatomiques et fonctionnelles de la reproduction chez le zébu. Rapport aux VIII Journées Médicales de Dakar, 1973. Rev. Elev. Méd. vét. Pays trop., 1973, 26 (4) : 21-48 a.

CUQ (P.). Particularités du fonctionnement ovarien dz la femelle zébu (Bos indicus) dans la zone soudanosahélienne de l'Afrique tropicale de l'Ouest. Communication au $58^{\mathrm{e}}$ Congrès de l'Association des Anatomistes, Liège, 1974. Bull. Ass. Anatomistes, 1975, 59: 139-144.

CUQ (P.), FERNEY (J.) et VAN CRAEYNEST (P.). Le cycle génital de la femelle zébu (Bos indicus) en zone soudano-sahélienne du Sénégal. Rev. Méd. vét., 1974, 37 : 147-173.

DENIS (J.-P.) et THIONGANE (A.I.). Analyse de la lactation de vaches pakistanaises au Sénégal. Rev. Elev. Méd. vét. Pays trop., 1974, 27 : 331-346.

DONALDSON (L. E.). Some observations on the fertility of beef cattle in Queensland. Aust. vet. J., 1962, 38: $447-454$.

PAPARELLA (G.). Physiologie et pathologie de la reproduction chez le zébu. Mémoire de maîtrise es-sciences vétérinaires, Alfort, 1974.

PLASSE (D.), WARNICK (A. C.) et KOGER (M.). Reproduction behaviour of Bos indicus females in a subtropical environment. IV. Length of cestrus cycle, duration of cestrus, time of ovulation in Brahman heifers. J. anim. Sci., 1970, 30: 63-71.

SHARMA (O. P.), TOMAR (N.S.) et SINGH (B. P.). Age and weight at puberty in Mariana heifers. Ind. $J$. dairy Sci, 1968, 21: 133-136.

WILLIS (M. B.) et WILSON (A.). Comparative reproductive performance of Brahman and Santa gertrudis cattle in a hot humid environment. I. Fertility and descriptive statistics. Am. Prod., 1974, 18: 35-42.

\section{INDEX}

\section{A}

Activité sexuelle continue, 337.

Activité sexuelle discontinue, 337, 347, 357, 358, 372.

Ampoule tubaire, 361, 362.

Angle caudal du ligament large, 374.

Angle cranio-dorsal du ligament large, 374.

Angle cranio-ventral du ligament large, 374.

Anœstrus, 337, 347, 357, 358, 372.

Anse cornuale, $369,373,384,385$.

Antrum voir cavité folliculaire, 348, 351.

Appendices vésiculaires, 360 .

Arcade veineuse dorsale de l'oviducte, 367.

Arcade veineuse vestibulo-vaginale, 395.

Artère du bulbe vestibulaire, 395 .

Artère cervico-utérine, 381.

Artère clitoridienne, 395 .

Artère dorsale du clitoris, 395.

Artère helicines, 347.

Artère honteuse interne, 393.

Artère ovarienne, 344.

Artère périnéale dorsale, 393.

Artère périnéale ventrale, 393.

Artère profonde du clitoris, 395.

Artère rectale moyenne, 395.

Artère rectale caudale, 393.

Artère salpingo-utérine, 365.

Artère tubo-utérine, 344, 365, 381.

Artère uréthrale, 393.

Artère utérine, 381.

Artère utérine caudale, 381 .

Artère vaginale, 381, 393, 395.

Artère vésicale, 395 .

Artère vestibulaire, 393.

\section{B}

Bord cranial du ligament large, 373.

Bord dorsal du ligament large, 373.

Bord dorsal ou mésovarien de l'ovaire, 339, 345.

Bord fixe ou mésométrique de l'utérus, 369.

Bord latéral du vagin, 386.

Bord libre de l'utérus. 369.

Bord ventral du ligament large, 373 .

Bord ventral ou libre de l'ovaire, 339.

Bourrelets circulaires du col utérin, 372.

Bourrelets vaginaux, 386 .

Bourse ovarique, $340,341,362$.

Bulbe vestibulaire, 390 .

\section{$\mathbf{C}$}

Canal cervical, 372, 380.

Canaux longitudinaux de l'époophoron ou canaux de Gärtner, 389.

Capacitation des spermatozoïdes, 363, 380.

Capuchon du clitoris, 391.

Caroncules, 371, 372, 378.

Cavité de la bourse ovarique, 341 .

Cavité des cornes utérines, 371.

Cavité du corps utérin, 371 .

Cavité folliculaire, $348,350,351$.

Cavité utérine, 371.

Cavité vaginale, 386 .

Cavité vestibulaire, 388.

Cellules absorbantes de l'épithélium tubaire, 363.

Cellules en batonnets de l'épithélium tubaire, 363 .

Cellules ciliées de l'épithélium tubaire, 363.

Cellules ciliées de l'épithélium utérin, 378. 
Cellules folliculeuses, 347, 348, 351.

Cellules glandulaires au repos de l'épithélium tubaire, 363.

Cellules intercalaires de l'épithélium utérin, 378, 380.

Cellules à mucus de l'épithélium vaginal, 387.

Cellules non ciliées de l'épithélium tubaire, 363 .

Cellules non ciliées de l'épithélium utérin, 378.

Cellules sécrétoires de l'épithélium tubaire, 363.

Cellules sécrétoires de l'épithélium utérin, 378.

Cellules thécales, 348.

Chaleurs, 349, 351.

Chaleurs anovulatoires, 350.

Chaleurs à durée prolongée, 350 .

Chaleurs durant la gestation, 358 .

Chaleurs silencieuses, 349.

Chorion de la muqueuse du canal cervical, 380 .

Chorion de la muqueuse caronculaire, 378.

Chorion de la muqueuse tubaire, 363.

Chorion de la muqueuse utérine, 378.

Chorion de la muqueuse vaginale, 387.

Chorion de la muqueuse vestibulaire, 390.

Clitoris, 391.

Cour uterin, 378.

Col uterin, 368, 371, 372, 377, 384.

Commissure dorsale de la vulve, 391 .

Commissure ventrale de la vulve, 391 .

Corne utérine, 368.

Corps blanc, 356.

Corps caverneux du clitoris, 392.

Corps du clitoris, 391.

Corps gestatif, 337, 347, 354, 356, 357.

Corps gestatif primaire, 357 .

Corps gestatif secondaire, 357 .

Corps gestatif de supplémentation, 357.

Corps gestatif tertiaire, 357.

Corps jaune, 337,356 .

Corps jaune accessoire, 357.

Corps jaune cyclique, 337, 354, 355.

Corps jaune extra-ovarien, 354 .

Corps jaune extra et intra ovarien, 354.

Corps jaune de gestation, 337, 356 .

Corps jaune intra-ovarien, 354.

Corps de l'oviducte, 361 .

Corps progestatifs, 337, 347, 354, 356.

Corps rouge, 354.

Corps utérin, 367.

Couche basale de l'épithélium vaginal, 387.

Couche conjonctive sous-épithéliale du stroma ovarien, 345 .

Couche externe de la thèque folliculaire, 348.

Couche granuleuse des follicules ovariens cavitaires, 348.

Couche intermédiaire de l'épithélium vaginal, 387.

Couche interne du myomètre, 376.

Couche interne de la thèque folliculaire, 348 .

Couche moyenne ou vasculaire du myomètre, 376.

Couche profonde du myomètre, 376 .

Couche sous-séreuse du vagin, 387.

Couche superficielle de l'épithélium vagınal, 387.

Couche superficielle du myomètre. 376 .

Couche vasculaire ou moyenne du myomètre, 376.

Couronne radiée, 348.

Couronne vaginale, 386.

Crypte caronculaire, 378.

Crypte utérin, 378.

Culs de sac pelviens du péritoine, 392.

Cul de sac recto-génital, 392.

Cul de sac vésico-génital, 392.

Cumulus oophorus voir massif germinal, 348.

Cycle ostral, 337, 340, 371.

\section{D}

Dimensions de l'ovaire, 339.

Di-cestrus, 337, 356, 372, 380, 388.
Disparition des corps gestatifs, 337, 358

Diverticule sous uréthral, 389.

\section{$\mathbf{E}$}

Endomètre, 378.

Eperon utérin, 371, 377.

Epithélium germinatif, 345.

Epithélium de la muqueuse tubaire, 363.

Epithélium de la muqueuse utérine, 378, 380.

Epithélium superficiel de l'ovaire, 345, 349, 357.

Epithélium tubaire, 363.

Epithélium vaginal, $387,388$.

Epithélium vestibulaire, 391.

Epithélium vulvaire, 391.

Espace rétro-péritonéal, 393.

Extrémité ovarienne de l'oviducte, 360, 361.

Extrémité tubaire de l'ovaire, 339.

Extrémité utérine de l'ovaire, 339.

\section{$\mathbf{F}$}

Face antrale ou latérale de l'ovaire, 339, 344, 345.

Face latérale du ligament large, 374.

Face médiale du ligament large, 374.

Face médiale ou viscérale de l'ovaire, 339, 344, 345.

Fente vulvaire, 391.

Follicule atrétique, $350,351$.

Follicule atrétique à dégénérescence épithéliale complète, 350.

Follicule atrétique à granulosa persistante, 351 .

Follicule atrétique à ovocyte persistant, 351 .

Follicules cavitaires, 348, 349, 357.

Follicules dégénératifs, 350.

Follicules déhiscents, 349 .

Follicules évolutifs, 347,350 .

Follicules involutifs, 347, 350.

Follicules kystoïdes, 351 .

Follicules lamelleux, 351.

Follicules mûrs, 348, 349.

Follicules ovariens à plusieurs ovocytes, 350 .

Follicules ovariens cavitaires, 348, 349.

Follicules ovariens en croissance, 348.

Follicules ovariens primaires, 347.

Follicules ovariens pleins, 347.

Follicules ovariens primordiaux, 347.

Fonction endocrine de l'ovaire, 337, 350.

Fonction gamétogène de l'ovaire, 337.

Fornix vaginal, 387, 392.

Fosse clitoridienne, 391.

Frange ovarienne du pavillon de l'oviducte, 341, 360, 362.

Franges du pavillon de l'oviducte, 360.

Frottis vaginaux, 388 .

\section{G}

Ganglions iliaques internes, 396.

Ganglions iliaques médiaux, 344, 367, 385, 396.

Ganglions mammaires, 396.

Gelatine de Warton, 373.

Gemellarité, 349.

Gestations extra-utérines, 361.

Gland du clitoris, 391, 392.

Glandes utérines, 380, 381.

Glandes vestibulaires, 390 .

Glandes vestibulaires accessoires, 390.

Glandes vestibulaires principales, 390.

Glandes follicules cavitaires, 348 .

Granulo-luteocytes, 356, 357.

Granulosa, 348, 351.

\section{H}

Hile de l'ovaire, 339.

Hymen, 386, 387, 388.

Hyperhémie de la muqueuse utérine, 376, 377, 381. 


\section{I}

Ilots progestatifs des corps jaunes, 356 .

Ilots thécaux des corps jaunes, 356.

Images pteridiennes, 388 .

Insémination artificielle, 350 .

Isthme tubaire, 361, 365.

\section{$\mathbf{J}$}

Jonction tubo-utérine, 361, 377, 378, 380, 381.

\section{$\mathbf{L}$}

Lame muqueuse épithéliale de l'oviducte, 363.

Lame muqueuse épithéliale de l'utérus, 378.

Lame muqueuse épithéliale du vagin, 387.

Lame muqueuse épithéliale du vestibule, 391.

Lame séreuse épithéliale de l'oviducte, 362.

Lame séreuse épithéliale de l'utérus, 376.

Lame séreuse épithéliale du vagin, 387.

Lame sous-séreuse de l'oviducte, 362.

Lame sous-séreuse de l'utérus, 376.

Lèvres de la vulve, 391.

Ligament inguinal, 374.

Ligament inter-cornual, 369.

Ligament large, 340, 373, 375 .

Ligament 'latéral de la vessie, 374, 375.

Ligament propre de l'ovaire, 341.

Ligament rond de l'utérus, 374.

Ligament suspenseur de l'ovaire, 341.

Ligament tubo-ovarique, $341,360,362$.

Ligament utéro-ovarien, 341.

Liquide folliculaire ou liqueur folliculaire, 348, 349, 351.

Loge sous-omentale de la cavité péritonéale, 375.

Loge sus-omentale de la cavité péritonéale, 375.

Luteocytes, 354.

Luteocytes thécaux, 356.

\section{M}

Macula pellucida, 349 .

Massif germinal, 348.

Méat uréthral on urinaire, 389.

Membrane pellucide, 348.

Mésomètre, 373.

Mésosalpinx, 340, 341, 360, 361, 373, 374.

Mésovarium, 340, 341, 373.

Mésovarium distal, 340, 373.

Mésovarium proximal, $340,373$.

Migration de l'cuf, 363.

Migration fotale de lovaire, 340.

Migration gravidique de l'ovaire, 342 .

Migration post-gravidique de l'ovaire, 342.

Mucus vaginal, 388.

Muqueuse de l'oviducte, 363.

Muqueuse de l'utérus, 378, 379, 380.

Muqueuse du vagin, 379, 387.

Muqueuse du vestibule vaginal, 390 .

Muqueuse de la vulve, 391.

Muscle constricteur du vestibule, 390.

Muscle constricteur de la vulve, 392.

Muscle ischio-cavernewx, 391.

Muscle rétracteur du clitoris, 392.

Musculaire de la séreuse utérine, 376.

Musculature propre de l'oviducte, 363.

Musculature sous-péritonéale de l'oviducte, 362.

Myomètre, 376.

\section{$\mathbf{N}$}

Nerf honteux interne, 396.

Nerfs de l'ovaire, $345,347$.

Nerfs de l'oviducte, 367 .
Nerfs de l'utérus, 385.

Nerfs de la portion copulatrice, 396.

\section{$\mathbf{0}$}

Estrus à chaleurs silencieuses, 349.

Estrus en cours de gestation, 357.

Organites de l'ovaire, 347.

Orifice abdominal de la trompe utérine, 360.

Orifice externe (ou vaginal) du col utérin, 372.

Orifice externe de l'urèthre, 389.

Orifice interne du col utérin, 372 .

Orifice utérin de l'oviducte, 362 .

Orifice vaginal, $387,388$.

Orifice vulvaire, 391 .

Ovaire, 337, 338, 339, 358, 359.

Oviducte, $360,366,381$.

Ovocyte, 347, 351.

Ovulation, $345,349,354$.

Ovule, 337, 349, 360.

Paramètre, 376.

Paroi dorsale du vagin, 386.

Paroi ventrale du vagin, 386 .

Pavillon de la trompe utérine, 360, 361, 363.

Périmètre, 376.

Périné, 393.

Période ou phase ostrogénique du cycle cestral, 337.

Période ou phase progestative du cycle ostral, 337.

Périodes de repos sexuel, 358.

Petits follicules cavitaires, 348.

Phase de dégénérescence des corps gestatifs, 357.

Phase de dégénérescence des corps progestatifs, 356.

Phase de développement des corps gestatifs, 356 .

Phase de développement des corps progestatifs, 354.

Placentation cotylédonaire du chorion embryonnaire, 381.

Placentation diffuse du chorion embryonnaire, 381.

Plexus artériolaire ovarien, 347.

Plexus hypogastrique, 385, 396.

Plexus oyarien, 345.

Plexus veineux de l'ovaire, 347.

Plis tubaires, $361,363$.

Poids de l'ovaire, 339.

Portion en anse de la veine marginale de l'utérus, 384.

Portion caudale (ou rectiligne) des cornes utérines, 368.

Portion caudale du vagin, 386-387.

Portion copulatrice des organes génitaux de la femelle, 386, 396.

Portion craniale (ou tubaire) des cornes utérines, 369.

Portion craniale du vagin, 386-387.

Portion gestative ou tubulaire des organes génitaux de Ia femelle, $360,386$.

Portion glandulaire des organes génitaux de la femelle, 337.

Portion moyenne des cornes utérines ou anse cornuale, 369,384 .

Portion plexiforme de la veine marginale de l'utérus, $384,385$.

Portion pré-vaginale du col utérin, 372.

Portion rectiligne de la veine marginale de l'utérus, 385.

Portion sinueuse de la veine marginale de l'utérus, 384.

Portion tubulaire (ou gestative) des organes génitaux de la femelle, 360,386 .

Portion utérine de l'oviducte, $361,363$.

Portion vaginale du col utérin, 372.

Post-œstrus, 337, 356, 373, 380, 388.

Prépuce du clitoris, 391 .

Pro-œestrus, 337, 356, 373, 380. 
Racines du clitoris, 391.

Rameau caudal de l'artère utérine, 384.

Rameau cranial de l'artère utérine, 381 .

Rameau moyen de l'artère utérine, 381.

Rameau utérin de l'artère ovarienne, 344, 365, 381.

Rameau utérin de l'artère vaginale, 381, 395 .

Rameau yeineux tubaire cranial, 367.

Rameaux labiaux de l'artère périnéale dorsale, 393.

Rameaux labiaux de l'artère périnéale ventrale, 393.

Rameaux labiaux de la veine honteuse interne, 395.

Rameaux mammaires de l'artère périnéale ventrale, 393.

Rameaux rectaux de la veine honteuse interne, 395.

Rameaux tubaires de l'artère ovarienne, 344, 365.

Rameaux tubaires caudal de l'artère ovarienne, 366.

Rameaux tubaires crânial de l'artère ovarienne, 365 .

Rameaux tubaires moyen de l'artère ovarienne, 365 .

Rameaux vaginaux de l'artère vaginale, 395.

Rameaux vaginaux de la veine honteuse interne, 395.

Rameaux vaginaux de la veine vaginale, 395.

Rameaux vestibulaires de la veine honteuse interne, 395.

Rameaux vestibulaires de la veine vaginale, 395.

Rapports des ovaires, 342.

Rapports des oviductes, 362.

Rapports de la portion copulatrice des organes génitaux, 392, 393.

Rapports de l'utérus, 375.

Région corticale de l'ovaire, 345.

Région médullaire de l'ovaire, 345.

Remplacement des corps gestatifs, 337, 357.

Repos sexuel, 337, 347, 358, 373.

Rete ovarii, 345,346 (fig. 4).

Sécrétion glandulaire de la muqueuse utérine, 380.

Sécrétion de surface de la muqueuse utéríne, 380 .

Sinus vestibulaire, 390 .

Stigma, 349.

Stroma ovarien, 345, 357.

Structure du ligament large, 375

Supplémentation des corps gestatıfs, 337, 357.

Surcharge lutéinique, 357.

Système anastomotique dorsal des rameaux tubaires, 365 .

Système anastomotique ventral des rameaux tubaires, 365.

Système nerveux autonome des ovaires, 345, 347.

Système nerveux autonome des oviductes, 367.

Système nerveux autonome de l'utérus, 385.

Système nerveux autonome de la portion copulatrice, 396.

\section{$\mathbf{T}$}

Tache translucide, 349.

Thèque externe, 348,349 .

Thèques folliculaires, 348 .

Thèque interne, $348,349,351$.

Topographie des ovaires, 342,345 .

Topographie des oviductes, 362.
Topographie de l'utérus, 375.

Trompes utérines, 360.

Tunique adventice du vagin, 387.

Tunique adventice du vestibule, 390 .

Tunique muqueuse du canal cervical, 380.

Tunique muqueuse des caroncules, 378.

Tunique muqueuse de la jonction tubo-utérine, 380 .

Tunique muqueuse de l'oviducte, 363.

Tunique muqueuse de l'utérus, 378, 380.

Tunique muqueuse du vagin, 387.

Tunique muqueuse du vestibule vaginal, 390.

Tunique musculeuse du col utérin, 377.

Tunique musculeuse de la jonction tubo-utérine, 377.

Tunique musculeuse de l'oviducte, 362.

Tunique musculeuse de l'utérus, 376.

Tunique musculeuse du vagin, 387 .

Tunique musculeuse du vestibule vaginal, 390.

Tunique séreuse de l'oviducte, 362 .

Tunique séreuse de l'utérus, 376.

Tunique séreuse du vagin, 387 .

\section{$\mathbf{U}$}

Utérus, 367

Utérus bipartitus, 367,385 .

$\mathbf{v}$

Vagin, 386.

Vaisseaux lymphatiques de l'ovaire, 344, 347.

Vaisseaux lymphatiques de l'oviducte, 367.

Vaisseaux lymphatiques de la portion copulatrice, 395.

Vaisseaux lymphatiques de l'utérus, 385.

Veine du bulbe vestibulaire, 395.

Veine clitoridienne, 395.

Veine dorsale du clitoris, 395.

Veine honteuse interne, 385, 395.

Veine infundibulaire, 367.

Veine marginale de l'utérus, 367, 384, 395.

Veine ovarienne, $344,347,385$.

Veine périnéale dorsale, 395.

Veine périnéale ventrale, 395.

Veine profonde du clitoris, 395.

Veine tubaire caudale, 367,385 .

Veine tubaire craniale, 367, 385.

Veine tubaire ventrale, 367.

Veine tubo-utérine, $367,385$.

Veine urétérale moyenne, 385.

Veine utérine, 385

Veine utérine craniale, 367.

Veine vaginale, 385,395

Veine vésicale, 395 .

Veine vestibulaire, 395.

Velum utérin, 371.

Vestibule vaginal, $388,390$.

Villosités du chorion embryonnaire, 381.

Vitrée folliculaire, 347.

Vulve, 391.

\section{$\mathbf{Z}$}

Zone parenchymateuse de l'ovaire, 345 .

Zone pellucide, 348 .

Zone de recouvrement péritonéal, 339, 345.

Zone vasculaire de l'ovaire, 346. 